\title{
MODEL HIGGS BUNDLES IN EXCEPTIONAL COMPONENTS OF THE $\operatorname{Sp}(4, \mathbb{R})$-CHARACTER VARIETY
}

\author{
GEORGIOS KYDONAKIS
}

\begin{abstract}
We establish a gluing construction for Higgs bundles over a connected sum of Riemann surfaces in terms of solutions to the $\operatorname{Sp}(4, \mathbb{R})$-Hitchin equations using the linearization of a relevant elliptic operator. The construction can be used to provide model Higgs bundles in all the $2 g-3$ exceptional components of the maximal $\mathrm{Sp}(4, \mathbb{R})$-Higgs bundle moduli space, which correspond to components solely consisted of Zariski dense representations. This also allows a comparison between the invariants for maximal Higgs bundles and the topological invariants for Anosov representations constructed by O. Guichard and A. Wienhard.
\end{abstract}

\section{INTRODUCTION}

Let $\Sigma$ be a closed connected and oriented surface of genus $g \geq 2$ and $G$ be a connected semisimple Lie group. The moduli space of reductive representations of $\pi_{1}(\Sigma)$ into $G$ modulo conjugation

$$
\mathcal{R}(G)=\operatorname{Hom}^{+}\left(\pi_{1}(\Sigma), G\right) / G
$$

has been an object of extensive study and interest. Fixing a complex structure $J$ on the surface $\Sigma$ transforms this into a Riemann surface $X=(\Sigma, J)$ and opens the way for holomorphic techniques using the theory of Higgs bundles. The non-abelian Hodge theory correspondence provides a real-analytic isomorphism between the character variety $\mathcal{R}(G)$ and the moduli space $\mathcal{M}(G)$ of polystable $G$-Higgs bundles. The case when $G=\operatorname{Sp}(4, \mathbb{R})$ has received considerable attention by many authors, who studied the geometry and topology of the moduli space $\mathcal{M}(\operatorname{Sp}(4, \mathbb{R}))$; see for instance [7], [10], [20]. The subspace of maximal $\operatorname{Sp}(4, \mathbb{R})$-Higgs bundles, $\mathcal{M}^{\text {max }}$, that is, the one containing Higgs bundles with extremal Toledo invariant, has been shown to have $3 \cdot 2^{2 g}+2 g-4$ connected components [19].

Among the connected components of $\mathcal{M}^{\text {max }}$, there are $2 g-3$ exceptional components of this moduli space. These components are all smooth but topologically non-trivial, and representations in these do not factor through any proper reductive subgroup of $\operatorname{Sp}(4, \mathbb{R})$, thus have Zariski-dense image in $\operatorname{Sp}(4, \mathbb{R})$. On the other hand, for the remaining $3 \cdot 2^{2 g}-1$ components, model Higgs bundles can be obtained by embedding stable $\mathrm{SL}(2, \mathbb{R})$-Higgs data into $\operatorname{Sp}(4, \mathbb{R})$, using appropriate embeddings $\phi: \operatorname{SL}(2, \mathbb{R}) \hookrightarrow \operatorname{Sp}(4, \mathbb{R})$ (see [7]). The construction of $\operatorname{Sp}(4, \mathbb{R})$-Higgs bundles that lie in the $2 g-3$ exceptional components is the principal objective in this article.

From the point of view of the character variety $\mathcal{R}^{\max }$, model representations in a subfamily of the $2 g-3$ special components have been effectively constructed by O. Guichard and A. Wienhard in [20] by amalgamating certain fundamental group representations defined over topological surfaces with one boundary component.

The first step in establishing a gluing construction from the holomorphic point of view is to describe holomorphic objects corresponding to $\mathrm{Sp}(4, \mathbb{R})$-representations over a surface

Date: 27 June 2018.

ISSN: 2347-1921

Journal: Journal of Advances in Mathematics 
with boundary with fixed arbitrary holonomy around the boundary. These objects are Higgs bundles defined over a Riemann surface with a divisor, together with a weighted flag on the fibers over the points in the divisor, namely parabolic $S p(4, \mathbb{R})$-Higgs bundles. As in the non-parabolic case, a notion of maximality can still be defined for these objects.

It is important that a gluing construction of parabolic Higgs bundles over the complex connected sum $X_{\#}$ of two distinct compact Riemann surfaces $X_{1}$ and $X_{2}$ with a divisor of $s$-many distinct points on each, is formulated so that the gluing of stable parabolic pairs is providing a polystable Higgs bundle over $X_{\#}$. Moreover, in order to construct new models in the components of $\mathcal{M}\left(X_{\#}, \operatorname{Sp}(4, \mathbb{R})\right)$, the parabolic gluing data over $X_{1}$ and $X_{2}$ are chosen to be coming from different embeddings of $\mathrm{SL}(2, \mathbb{R})$-parabolic data into $\mathrm{Sp}(4, \mathbb{R})$, and so a priori do not agree over disks around the points in the divisors. We choose to switch to the language of solutions to Hitchin's equations and make use of the analytic techniques of C. Taubes for gluing instantons over 4-manifolds [37] in order to control the stability condition. These techniques have been applied to establish similar gluing constructions for solutions to gauge-theoretic equations, see for instance [13], [14], [21], [30].

The problem involves perturbing the initial data into model solutions which are identified locally over the annuli around the points in the divisors, thus allowing the construction of a pair over $X_{\#}$ that combines initial data over $X_{1}$ and $X_{2}$. The existence of these perturbations in terms of appropriate gauge transformations is provided for $\operatorname{SL}(2, \mathbb{R})$-data, and then we use the embeddings of $\mathrm{SL}(2, \mathbb{R})$ into $\operatorname{Sp}(4, \mathbb{R})$ to extend this deformation argument for our initial pairs. This produces an approximate solution to the $\operatorname{Sp}(4, \mathbb{R})$-Hitchin equations $\left(A_{R}^{a p p}, \Phi_{R}^{a p p}\right)$ over $X_{\#}$, with respect to a parameter $R>0$ which describes the size of the neck region in the construction of $X_{\#}$. The pair $\left(A_{R}^{a p p}, \Phi_{R}^{a p p}\right)$ coincides with the initial data over each hand side Riemann surface and with the model solution over the neck region.

The next step is to correct this approximate solution to an exact solution of the $\operatorname{Sp}(4, \mathbb{R})$ Hitchin equations over the complex connected sum of Riemann surfaces. In other words, we seek for a complex gauge transformation $g$ such that $g^{*}\left(A_{R}^{a p p}, \Phi_{R}^{a p p}\right)$ is an exact solution of the $\mathrm{Sp}(4, \mathbb{R})$-Hitchin equations. The argument providing the existence of such a gauge is translated into a Banach fixed point theorem argument and involves the study of the linearization of a relevant elliptic operator. For Higgs bundles this was first studied by R. Mazzeo, J. Swoboda, H. Weiss and F. Witt in [25], who described solutions to the $\operatorname{SL}(2, \mathbb{C})$-Hitchin equations near the ends of the moduli space. A crucial step in this argument is to show that the linearization of the $G$-Hitchin operator at our approximate solution $\left(A_{R}^{a p p}, \Phi_{R}^{a p p}\right)$ is invertible; this is obtained by showing that an appropriate self-adjoint Dirac-type operator has no small eigenvalues. This method was also used by J. Swoboda in [36] to produce a family of smooth solutions of the $\mathrm{SL}(2, \mathbb{C})$-Hitchin equations, which may be viewed as desingularizing a solution with logarithmic singularities over a noded Riemann surface. The analytic techniques from [36], are extended to provide the main theorem from that article for solutions of the $\operatorname{Sp}(4, \mathbb{R})$ Hitchin equations as well, and moreover to obtain our main result:

Theorem 1.1. Let $X_{1}$ be a closed Riemann surface of genus $g_{1}$ and $D_{1}=\left\{p_{1}, \ldots, p_{s}\right\}$ be a collection of s-many distinct points on $X_{1}$. Consider respectively a closed Riemann surface $X_{2}$ of genus $g_{2}$ and a collection of also $s$-many distinct points $D_{2}=\left\{q_{1}, \ldots, q_{s}\right\}$ on $X_{2}$. Let $\left(E_{1}, \Phi_{1}\right) \rightarrow X_{1}$ and $\left(E_{2}, \Phi_{2}\right) \rightarrow X_{2}$ be parabolic stable $S p(4, \mathbb{R})$-Higgs bundles with corresponding solutions to the Hitchin equations $\left(A_{1}, \Phi_{1}\right)$ and $\left(A_{2}, \Phi_{2}\right)$. Assume that these solutions agree with model solutions $\left(A_{1, p_{i}}^{\bmod }, \Phi_{1, p_{i}}^{\bmod }\right)$ and $\left(A_{2, q_{j}}^{\bmod }, \Phi_{2, q_{j}}^{\bmod }\right)$ near the points $p_{i} \in$ $D_{1}$ and $q_{j} \in D_{2}$, and that the model solutions satisfy $\left(A_{1, p_{i}}^{\bmod }, \Phi_{1, p_{i}}^{\bmod }\right)=-\left(A_{2, q_{j}}^{\bmod }, \Phi_{2, q_{j}}^{\bmod }\right)$, 
for $s$-many possible pairs of points $\left(p_{i}, q_{j}\right)$. Then there is a polystable $S p(4, \mathbb{R})$-Higgs bundle $\left(E_{\#}, \Phi_{\#}\right) \rightarrow X_{\#}$, constructed over the connected sum of Riemann surfaces $X_{\#}=X_{1} \# X_{2}$ of genus $g_{1}+g_{2}+s-1$, which agrees with the initial data over $X_{\#} \backslash X_{1}$ and $X_{\#} \backslash X_{2}$.

By analogy with the terminology introduced by O. Guichard and A. Wienhard in their construction of hybrid representations, we call the polystable Higgs bundles corresponding to such exact solutions hybrid. The construction can have a wider applicability for identifying smooth points in moduli of Higgs bundles. As one application, we build Higgs bundles corresponding to Zariski dense representations into $\mathrm{Sp}(4, \mathbb{R})$. For this purpose, we look at how the Higgs bundle topological invariants behave under the complex connected sum operation. We first show the following:

Proposition 1.2. Let $X_{\#}=X_{1} \# X_{2}$ be the complex connected sum of two closed Riemann surfaces $X_{1}$ and $X_{2}$ with divisors $D_{1}$ and $D_{2}$ of s-many distinct points on each surface, and let $V_{1}, V_{2}$ be parabolic principal $H^{\mathbb{C}}$-bundles over $X_{1}$ and $X_{2}$ respectively. For a parabolic subgroup $P \subset H^{\mathbb{C}}$, a holomorphic reduction $\sigma$ of the structure group of $E$ from $H^{\mathbb{C}}$ to $P$ and an antidominant character $\chi$ of $P$, the following identity holds:

$$
\operatorname{deg}\left(V_{1} \# V_{2}\right)(\sigma, \chi)=\operatorname{pardeg}_{\alpha_{1}}\left(V_{1}\right)(\sigma, \chi)+\operatorname{pardeg}_{\alpha_{2}}\left(V_{2}\right)(\sigma, \chi) .
$$

Note that an analogous additivity property for the Toledo invariant was established by M. Burger, A. Iozzi and A. Wienhard in [8] from the point of view of fundamental group representations. It implies in particular that the connected sum of maximal parabolic $G$ Higgs bundles is again a maximal (non-parabolic) $G$-Higgs bundle.

We find model Higgs bundles in all exceptional components of the maximal $\operatorname{Sp}(4, \mathbb{R})$-Higgs bundle moduli space; these models are described by hybrid Higgs bundles. In the case when $G=\operatorname{Sp}(4, \mathbb{R})$, considering all possible decompositions of a surface $\Sigma$ along a simple, closed, separating geodesic is sufficient in order to obtain representations in the desired components of $\mathcal{M}^{\text {max }}$, which are fully distinguished by the calculation of the degree of a line bundle. This degree equals the Euler class for a hybrid representation as defined by O. Guichard and A. Wienhard, although these invariants live naturally in different cohomology groups.

Acknowledgments. This work was part of the author's requirements for the $\mathrm{Ph}$. D. degree at the University of Illinois at Urbana-Champaign. I am particularly grateful to my doctorate advisor, Professor Steven Bradlow, for his continuous support and guidance towards the completion of this project, as well as to Indranil Biswas, Olivier Guichard, Jan Swoboda, Nicolaus Treib, Hartmut Weiss and Richard Wentworth for shared insights. A very special thanks to Rafe Mazzeo for a series of illuminating discussions and a wonderful hospitality during a visit to Stanford University in April 2016. The author acknowledges support from U.S. National Science Foundation grants DMS 1107452, 1107263, 1107367 "RNMS: GEometric structures And Representation varieties" (the GEAR Network).

\section{2. $\operatorname{Sp}(4, \mathbb{R})$-Higgs Bundles And Surface group REPRESEntations}

2.1. Moduli spaces of $G$-Higgs bundles. Let $X$ be a compact Riemann surface and let $G$ be a real reductive group. The latter involves considering $\operatorname{Cartan}$ data $(G, H, \theta, B)$, where $H \subset G$ is a maximal compact subgroup, $\theta: \mathfrak{g} \rightarrow \mathfrak{g}$ is a Cartan involution and $B$ is a non-degenerate bilinear form on $\mathfrak{g}$, which is $\operatorname{Ad}(G)$-invariant and $\theta$-invariant. The Cartan involution $\theta$ gives a decomposition (called the Cartan decomposition)

$$
\mathfrak{g}=\mathfrak{h} \oplus \mathfrak{m}
$$


into its \pm 1 -eigenspaces, where $\mathfrak{h}$ is the Lie algebra of $H$.

Let $H^{\mathbb{C}}$ be the complexification of $H$ and let $\mathfrak{g}^{\mathbb{C}}=\mathfrak{h}^{\mathbb{C}} \oplus \mathfrak{m}^{\mathbb{C}}$ be the complexification of the Cartan decomposition. The adjoint action of $G$ on $\mathfrak{g}$ restricts to give a representation (the isotropy representation) of $H$ on $\mathfrak{m}$. This is independent of the choice of Cartan decomposition, since any two Cartan decompositions of $G$ are related by a conjugation, using also that $[\mathfrak{h}, \mathfrak{m}] \subseteq \mathfrak{m}$, and the same is true for the complexified isotropy representation $\iota: H^{\mathbb{C}} \rightarrow \operatorname{GL}\left(\mathfrak{m}^{\mathbb{C}}\right)$ This introduces the following definition:

Definition 2.1. Let $K$ be the canonical line bundle over $X$. A $G$-Higgs bundle is a pair $(E, \varphi)$ where

- $E$ is a principal holomorphic $H^{\mathbb{C}}$-bundle over $X$ and

- $\varphi$ is a holomorphic section of the vector bundle $E\left(\mathfrak{m}^{\mathbb{C}}\right) \otimes K=\left(E \times{ }_{\iota} \mathfrak{m}^{\mathbb{C}}\right) \otimes K$

The section $\varphi$ is called the Higgs field.

Two $G$-Higgs bundles $(E, \varphi)$ and $\left(E^{\prime}, \varphi^{\prime}\right)$ are said to be isomoprhic if there is a vector bundle isomorphism $E \cong E^{\prime}$ which takes the induced $\varphi$ to $\varphi^{\prime}$ under the induced isomorphism $E\left(\mathfrak{m}^{\mathbb{C}}\right) \cong E^{\prime}\left(\mathfrak{m}^{\mathbb{C}}\right)$.

To define a moduli space of $G$-Higgs bundles we need to consider a notion of semistability, stability and polystability. These notions are defined in terms of an antidominant character for a parabolic subgroup $P_{A} \subseteq H^{\mathbb{C}}$ and a holomorphic reduction $\sigma$ of the structure group of the bundle $E$ from $H^{\mathbb{C}}$ to $P_{A}$ (see [16] for the precise definitions).

When the group $G$ is connected, principal $H^{\mathbb{C}}$-bundles $E$ are topologically classified by a characteristic class $c(E) \in H^{2}\left(X, \pi_{1}\left(H^{\mathbb{C}}\right)\right)=\pi_{1}\left(H^{\mathbb{C}}\right)=\pi_{1}(H)=\pi_{1}(G)$.

Definition 2.2. For a fixed class $d \in \pi_{1}(G)$, the moduli space of polystable G-Higgs bundles with respect to the group of complex gauge transformations is defined as the set of isomorphism classes of polystable $G$-Higgs bundles $(E, \varphi)$ such that $c(E)=d$. We will denote this set by $\mathcal{M}_{d}(G)$ and when the group $G$ is compact, the moduli space $\mathcal{M}_{d}(G)$ coincides with $\mathcal{M}_{d}\left(G^{\mathbb{C}}\right)$.

Using the general GIT constructions of A. Schmitt for decorated principal bundles in the case of a real form of a complex reductive algebraic Lie group it is shown that the moduli space $\mathcal{M}_{d}(G)$ is a complex analytic variety, which is algebraic when $G$ is algebraic. The expected dimension of the moduli space of $G$-Higgs bundles is $(g-1) \operatorname{dim} G^{\mathbb{C}}$, in the case when $G$ is a connected semisimple real Lie group; see [16], [31], [32] for details.

2.2. $G$-Hitchin equations. Let $(E, \varphi)$ be a $G$-Higgs bundle over a compact Riemann surface $X$. By a slight abuse of notation we shall denote the underlying smooth objects of $E$ and $\varphi$ by the same symbols. The Higgs field can be thus viewed as a $(1,0)$-form $\varphi \in \Omega^{1,0}\left(E\left(\mathfrak{m}^{\mathbb{C}}\right)\right)$. Given a reduction $h$ of structure group to $H$ in the smooth $H^{\mathbb{C}}$-bundle E, we denote by $F_{h}$ the curvature of the unique connection compatible with $h$ and the holomorphic structure on $E$. Let $\tau_{h}: \Omega^{1,0}\left(E\left(\mathfrak{g}^{\mathbb{C}}\right)\right) \rightarrow \Omega^{0,1}\left(E\left(\mathfrak{g}^{\mathbb{C}}\right)\right)$ be defined by the compact conjugation of $\mathfrak{g}^{\mathbb{C}}$ which is given fiberwise by the reduction $h$, combined with complex conjugation on complex 1-forms. The next theorem was proved in [16] for an arbitrary reductive real Lie group $G$.

Theorem 2.3. There exists a reduction $h$ of the structure group of $E$ from $H^{\mathbb{C}}$ to $H$ satisfying the Hitchin equation

$$
F_{h}-\left[\varphi, \tau_{h}(\varphi)\right]=0
$$

if and only if $(E, \varphi)$ is polystable. 
From the point of view of moduli spaces it is convenient to fix a $C^{\infty}$ principal $H$-bundle $\mathbf{E}_{H}$ with fixed topological class $d \in \pi_{1}(H)$ and study the moduli space of solutions to Hitchin's equations for a pair $(A, \varphi)$ consisting of an $H$-connection $A$ and $\varphi \in \Omega^{1,0}\left(X, \mathbf{E}_{H}\left(\mathfrak{m}^{\mathbb{C}}\right)\right)$ :

$$
\begin{aligned}
F_{A}-[\varphi, \tau(\varphi)] & =0 \\
\bar{\partial}_{A} \varphi & =0
\end{aligned}
$$

where $d_{A}$ is the covariant derivative associated to $A$ and $\bar{\partial}_{A}$ is the $(0,1)$-part of $d_{A}$, defining the holomorphic structure on $\mathbf{E}_{H}$. Also, $\tau$ is defined by the fixed reduction of structure group $\mathbf{E}_{H} \hookrightarrow \mathbf{E}_{H}\left(H^{\mathbb{C}}\right)$. The gauge group $\mathcal{G}_{H}$ of $\mathbf{E}_{H}$ acts on the space of solutions by conjugation and the moduli space of solutions is defined by

$$
\mathcal{M}_{d}^{\text {gauge }}(G):=\{(A, \varphi) \text { satisfying }(2.1) \text { and }(2.2)\} / \mathcal{G}_{H}
$$

Now, Theorem 2.3 implies that there is a homeomorphism

$$
\mathcal{M}_{d}(G) \cong \mathcal{M}_{d}^{\text {gauge }}(G)
$$

Using the one-to-one correspondence between $H$-connections on $\mathbf{E}_{H}$ and $\bar{\partial}$-operators on $\mathbf{E}_{H^{\mathbb{C}}}$, the homeomorphism in the above theorem can be interpreted by saying that in the $\mathcal{G}_{H}^{\mathbb{C}}$-orbit of a polystable $G$-Higgs bundle $\left(\bar{\partial}_{E_{0}}, \varphi_{0}\right)$ we can find another Higgs bundle $\left(\bar{\partial}_{E}, \varphi\right)$ whose corresponding pair $\left(d_{A}, \varphi\right)$ satisfies the equation $F_{A}-[\varphi, \tau(\varphi)]=0$, and this is unique up to $H$-gauge transformations.

2.3. Surface group representations. Let $\Sigma$ be a closed oriented (topological) surface of genus $g$. The fundamental group of $\Sigma$ is described by the presentation

$$
\pi_{1}(\Sigma)=\left\langle a_{1}, b_{1}, \ldots, a_{g}, b_{g} \mid \prod\left[a_{i}, b_{i}\right]=1\right\rangle
$$

where $\left[a_{i}, b_{i}\right]=a_{i} b_{i} a_{i}^{-1} b_{i}^{-1}$ is the commutator. Define the moduli space of reductive representations of $\pi_{1}(\Sigma)$ into $G$ modulo conjugation to be the orbit space

$$
\mathcal{R}(G)=\operatorname{Hom}^{\mathrm{red}}\left(\pi_{1}(\Sigma), G\right) / G
$$

This space is a real analytic variety and so $\mathcal{R}(G)$ is usually called the character variety (see $[18])$.

We can assign a topological invariant to a representation $\rho \in \mathcal{R}(G)$, by considering its corresponding flat $G$-bundle on $\Sigma$, defined as $E_{\rho}=\tilde{\Sigma} \times{ }_{\rho} G$. Here $\tilde{\Sigma} \rightarrow \Sigma$ is the universal cover and $\pi_{1}(\Sigma)$ acts on $G$ via $\rho$. A topological invariant is then given by the characteristic class $c(\rho):=c\left(E_{\rho}\right) \in \pi_{1}(G) \simeq \pi_{1}(H)$, for $H \subseteq G$ a maximal compact subgroup of $G$. For a fixed $d \in \pi_{1}(G)$ the moduli space of reductive representations with fixed topological invariant $d$ is now defined as the subvariety

$$
\mathcal{R}_{d}(G):=\{[\rho] \in \mathcal{R}(G) \mid c(\rho)=d\}
$$

Equipping the surface $\Sigma$ with a complex structure $J$, there corresponds to a reductive fundamental group representation a polystable $G$-Higgs bundle over the Riemann surface $X=$ $(\Sigma, J)$. This is seen using that any solution $h$ to Hitchin's equations defines a flat reductive $G$-connection

$$
D=D_{h}+\varphi-\tau(\varphi),
$$

where $D_{h}$ is the unique $H$-connection on $E$ compatible with its holomorphic structure. Conversely, given a flat reductive connection $D$ in a $G$-bundle $E_{G}$, there exists a harmonic metric, i.e. a reduction of structure group to $H \subset G$ corresponding to a harmonic section of $E_{G} / H \rightarrow X$. This reduction produces a solution to Hitchin's equations such that Equation 
(2.3) holds. In summary, we have the following seminal result, the non-abelian Hodge correspondence; its proof is based on combined work by N. Hitchin [22], C. Simpson [33], [35], S. Donaldson [12] and K. Corlette [11]:

Theorem 2.4. Let $G$ be a connected semisimple real Lie group with maximal compact subgroup $H \subseteq G$ and let $d \in \pi_{1}(G) \simeq \pi_{1}(H)$. Then there exists a homeomorphism

$$
\mathcal{R}_{d}(G) \cong \mathcal{M}_{d}(G)
$$

2.4. $\mathbf{S p}(4, \mathbb{R})$-Higgs bundles. In this article, we are particularly interested in the case when the group $G$ is the semisimple real subgroup of $\operatorname{SL}(4, \mathbb{R})$ that preserves a symplectic form on $\mathbb{R}^{4}$ :

where $J=\left(\begin{array}{cc}0 & I_{2} \\ -I_{2} & 0\end{array}\right)$ defines a symplectic form on $\mathbb{R}^{4}$, for $I_{2}$ the $2 \times 2$ identity matrix.

The Cartan involution $\theta: \mathfrak{s p}(4, \mathbb{C}) \rightarrow \mathfrak{s p}(4, \mathbb{C})$ with $\theta(X)=-X^{T}$ determines a Cartan decomposition for a choice of maximal compact subgroup $H \simeq \mathrm{U}(2) \subset \operatorname{Sp}(4, \mathbb{R})$ as follows

$$
\mathfrak{s p}(4, \mathbb{R})=\mathfrak{u}(2) \oplus \mathfrak{m}
$$

with complexification $\mathfrak{s p}(4, \mathbb{C})=\mathfrak{g l}(2, \mathbb{C}) \oplus \mathfrak{m}^{\mathbb{C}}$. It is shown in [15] that the general definition for a $G$-Higgs bundle specializes to the following:

Definition 2.5. An $S p(4, \mathbb{R})$-Higgs bundle over a compact Riemann surface $X$ is defined by a triple $(V, \beta, \gamma)$, where $V$ is a rank 2 holomorphic vector bundle over $X$ and $\beta, \gamma$ are symmetric homomorphisms $\beta: V^{*} \rightarrow V \otimes K$ and $\gamma: V \rightarrow V^{*} \otimes K$, where $K$ is the canonical line bundle over $X$.

2.5. $\mathbf{S p}(4, \mathbb{R})$-Hitchin equations. For the complexified Lie algebra $\mathfrak{s p}(4, \mathbb{C})$, notice that the involution $\sigma: \mathfrak{s p}(4, \mathbb{C}) \rightarrow \mathfrak{s p}(4, \mathbb{C}), \sigma(X)=\bar{X}$ defines the split real form

$$
\mathfrak{s p}(4, \mathbb{R})=\{X \in \mathfrak{s p}(4, \mathbb{C}) \mid \sigma(X)=X\},
$$

while the involution $\tau: \mathfrak{s p}(4, \mathbb{C}) \rightarrow \mathfrak{s p}(4, \mathbb{C}), \tau(X)=-X^{*}$ defines the compact real form

$$
\mathfrak{s p}(2)=\mathfrak{s p}(4, \mathbb{C}) \cap \mathfrak{u}(4)=\{X \in \mathfrak{s p}(4, \mathbb{C}) \mid \tau(X)=X\} .
$$

Since $\tau$ and the Cartan involution commute, we have $\tau\left(\mathfrak{m}^{\mathbb{C}}\right) \subseteq \mathfrak{m}^{\mathbb{C}}$ and then $\tau$ preserves the Cartan decomposition $\mathfrak{s p}(4, \mathbb{C})=\mathfrak{g l}(2, \mathbb{C}) \oplus \mathfrak{m}^{\mathbb{C}}$. Thus, there is an induced real form on $E\left(\mathfrak{m}^{\mathbb{C}}\right)$ which we shall call $\tau$ as well for simplicity. Now, it makes sense to apply $\tau$ on a section $\varphi \in \Omega^{1,0}\left(E\left(\mathfrak{m}^{\mathbb{C}}\right)\right)$.

Moreover, for $\varphi=\left(\begin{array}{ll}0 & \beta \\ \gamma & 0\end{array}\right)$ notice that

$$
-[\varphi, \tau(\varphi)]=\left[\varphi, \varphi^{*}\right]=\left(\begin{array}{cc}
\beta \bar{\beta}-\bar{\gamma} \gamma & 0 \\
0 & \gamma \bar{\gamma}-\bar{\beta} \beta
\end{array}\right)
$$

The $G$-Hitchin equations for $G=\operatorname{Sp}(4, \mathbb{R})$ with maximal compact subgroup $H \simeq \mathrm{U}(2) \subset$ $\operatorname{Sp}(4, \mathbb{R}) \operatorname{read}$

$$
\begin{aligned}
F_{A}-[\varphi, \tau(\varphi)] & =0 \\
\bar{\partial}_{A} \varphi & =0
\end{aligned}
$$

where:

- $A$ is a $\mathrm{U}(2)$-connection on a fixed smooth principal $\mathrm{U}(2)$-bundle $\mathbb{E}_{H} \rightarrow X$ 
- $\varphi \in \Omega^{1}\left(X, E_{H^{\mathbb{C}}}\left(\mathfrak{m}^{\mathbb{C}}\right)\right)$

- $\tau: \Omega^{1}\left(X, E_{H^{\mathbb{C}}}\left(\mathfrak{m}^{\mathbb{C}}\right)\right) \rightarrow \Omega^{1}\left(X, E_{H^{\mathbb{C}}}\left(\mathfrak{m}^{\mathbb{C}}\right)\right)$ is the compact real structure considered above.

- $\bar{\partial}_{A}$ is the $(0,1)$-part of the covariant derivative associated to $A$. whereas $\mathcal{G}_{H}=\operatorname{Aut}\left(\mathbb{E}_{H}\right)=\Omega^{0}\left(X, \mathbb{E}_{H} \times_{A d} H\right)$ is the gauge group of $\left(\mathbb{E}_{H}, h\right)$ for $H=\mathrm{U}(2)$.

2.6. The Toledo invariant and Cayley partner. A basic topological invariant for an $\operatorname{Sp}(4, \mathbb{R})$-Higgs bundle $(V, \beta, \gamma)$ is given by the degree of the underlying bundle

$$
d=\operatorname{deg}(V)
$$

This invariant, called the Toledo invariant labels only partially the connected components of the moduli space $\mathcal{M}(\operatorname{Sp}(4, \mathbb{R}))$. We use the notation $\mathcal{M}_{d}=\mathcal{M}_{d}(\operatorname{Sp}(4, \mathbb{R}))$ to denote the moduli space parameterizing isomorphism classes of polystable $\operatorname{Sp}(4, \mathbb{R})$-Higgs bundles with $\operatorname{deg}(V)=d$. The sharp bound below for the Toledo invariant when $G=\operatorname{Sp}(4, \mathbb{R})$ was first given by V. Turaev [38]:

Proposition 2.6. (Milnor-Wood inequality) Let $(V, \beta, \gamma)$ be a semistable $S p(4, \mathbb{R})$-Higgs bundle. Then $|d| \leq 2 g-2$.

Definition 2.7. We shall call $\operatorname{Sp}(4, \mathbb{R})$-Higgs bundles with Toledo invariant $d=2 g-2$ maximal and denote the components of $\mathcal{M}(\operatorname{Sp}(4, \mathbb{R}))$ with maximal positive Toledo invariant by $\mathcal{M}^{\max } \simeq \mathcal{M}_{2 g-2}$.

The proof of Proposition 2.6 given by P. Gothen in [19] in the language of Higgs bundles opens the way to considering new topological invariants for our Higgs bundles in order to count the exact number of components of $\mathcal{M}^{\max }$. Namely, one sees from that proof that for a maximal semistable $\operatorname{Sp}(4, \mathbb{R})$-Higgs bundle $(V, \beta, \gamma)$, the map $\gamma: V \rightarrow V^{*} \otimes K$ is an isomorphism.

For a fixed square root of the canonical bundle $K$, that is, a line bundle $L_{0}$ such that $L_{0}^{2}=K$, the isomorphism $\gamma$ can be used to construct an $\mathrm{O}(2, \mathbb{C})$-holomorphic bundle $\left(W, q_{W}\right)$, with $W:=V^{*} \otimes L_{0}$ and $q_{W}:=\gamma \otimes I_{L_{0}^{-1}}: W^{*} \stackrel{\simeq}{\rightarrow} W$. The Stiefel-Whitney classes of this orthogonal bundle $\left(W, q_{W}\right)$, which is called the Cayley partner of the $\operatorname{Sp}(4, \mathbb{R})$-Higgs bundle $(V, \beta, \gamma)$, are now appropriate topological invariants to study the topology of the moduli space $\mathcal{M}^{\text {max }}$; see [15] for more details.

2.7. The components of $\mathcal{M}^{\max }(X, \mathbf{S p}(\mathbf{4}, \mathbb{R}))$. Let $w_{i}\left(W, q_{W}\right) \in H^{i}(X, \mathbb{Z} / 2)$, for $i=1,2$ be the Stiefel-Whitney classes introduced in the previous subsection. The classification of $\mathrm{O}(2, \mathbb{C})$-holomorphic bundles by D. Mumford [26] provides that for a rank 2 orthogonal bundle $\left(W, q_{W}\right)$ with $w_{1}\left(W, q_{W}\right)=0$, then $W=L \oplus L^{-1}$, where $L \rightarrow X$ is a line bundle, and $q_{W}=\left(\begin{array}{ll}0 & 1 \\ 1 & 0\end{array}\right)$. The induced Higgs field on $W$ is a map

$$
\begin{aligned}
\theta: W & \rightarrow W \otimes K^{2} \\
L \oplus L^{-1} & \rightarrow\left(L \oplus L^{-1}\right) \otimes K^{2}
\end{aligned}
$$

and one can see that this $\theta$ induces a non-zero holomorphic map $L \rightarrow L^{-1} K^{2}$, otherwise the subbundle $L \subset W$ would violate stability for $\theta$. Thus, global sections of the bundle $L^{-2} K^{2}$ exist, that means, $\operatorname{deg}\left(L^{-2} K^{2}\right) \geq 0$ and so

$$
0 \leq \operatorname{deg}(L) \leq 2 g-2
$$


The degree $\operatorname{deg}(L)$ introduces an extra invariant for the study of components of the moduli space $\mathcal{M}^{\max }$. In fact, when $\operatorname{deg}(L)=2 g-2$ the connected components are parameterized by spin structures on the surface $\Sigma$. Using Morse theory techniques and a careful study of the closed subvarieties corresponding to all possible values of the invariants $w_{1}, w_{2}$ and $\operatorname{deg}(L)$, it was shown in [19] that the total number of connected components of the moduli space $\mathcal{M}^{\max }$ is $3 \cdot 2^{2 g}+2 g-4$, for $g$ the genus of the Riemann surface $X$.

2.8. Maximal fundamental group representations into $\mathbf{S p}(4, \mathbb{R})$. From an alternative point of view, the non-abelian Hodge theorem provides a homeomorphism of $\mathcal{M}^{\text {max }}$ to a moduli space of representations $\mathcal{R}^{\max }$, which we briefly introduce next:

Let $G$ be a Hermitian Lie group of non-compact type, that is, the symmetric space associated to $G$ is an irreducible Hermitian symmetric space of non-compact type. Using the identification $H^{2}\left(\pi_{1}(\Sigma), \mathbb{R}\right) \simeq H^{2}(\Sigma, \mathbb{R})$, the Toledo invariant of a representation $\rho: \pi_{1}(\Sigma) \rightarrow G$ is defined as the integer

$$
T_{\rho}:=\left\langle\rho^{*}\left(\kappa_{G}\right),[\Sigma]\right\rangle,
$$

where $\rho^{*}\left(\kappa_{G}\right)$ is the pullback of the Kähler class $\kappa_{G} \in H_{c}^{2}(G, \mathbb{R})$ of $G$ and $[\Sigma] \in H_{2}(\Sigma, \mathbb{R})$ is the orientation class. It is bounded in absolute value, $\left|T_{\rho}\right| \leq-C(G) \chi(\Sigma)$, where $C(G)$ is an explicit constant depending only on $G$; we refer the reader to [8] for more details.

Definition 2.8. A representation $\rho: \pi_{1}(\Sigma) \rightarrow G$ is called maximal whenever the Toledo invariant $T_{\rho}=-C(G) \chi(\Sigma)$.

Theorem 2.9 (S. Bradlow, O. García-Prada, P. Gothen [7]). There are $2 g-3$ connected components of $\mathcal{M}^{\max } \simeq \mathcal{R}^{\max }$, in which the corresponding representations do not factor through any proper reductive subgroup of $S p(4, \mathbb{R})$, thus they have Zariski-dense image in $S p(4, \mathbb{R})$.

In [20], O. Guichard and A. Wienhard describe model maximal fundamental group representations $\rho: \pi_{1}(\Sigma) \rightarrow \operatorname{Sp}(4, \mathbb{R})$ in the components of $\mathcal{R}^{\max }$. These models are distinguished into two subcategories, standard representations and hybrid representations.

We review the construction of these model representations in further detail with particular notice towards the construction of these hybrid representations. Fix a discrete embedding $i: \pi_{1}(\Sigma) \rightarrow \mathrm{SL}(2, \mathbb{R})$.

i) Irreducible Fuchsian representations

Choose the symplectic identification $\left(\mathbb{R}_{3}[X, Y],-\omega_{2}\right) \cong\left(\mathbb{R}^{4}, \omega\right)$ given by $X^{3}=e_{1}, X^{2} Y=$ $-e_{2}, Y^{3}=-e_{3}, X Y^{2}=\frac{-e_{4}}{\sqrt{3}}$, where $\omega$ is the symplectic form given by the antisymmetric matrix $J=\left(\begin{array}{cc}0 & \mathrm{Id}_{n} \\ -\mathrm{Id}_{n} & 0\end{array}\right)$. With respect to this identification the irreducible representation $\phi_{\text {irr }}: \mathrm{SL}(2, \mathbb{R}) \rightarrow \mathrm{Sp}(4, \mathbb{R})$ is given by

$$
\phi_{\text {irr }}\left(\begin{array}{ll}
a & b \\
c & d
\end{array}\right)=\left(\begin{array}{cccc}
a^{3} & -\sqrt{3} a^{2} b & -b^{3} & -\sqrt{3} a b^{2} \\
-\sqrt{3} a^{2} c & 2 a b c+a^{2} d & \sqrt{3} b^{2} d & 2 a b d+b^{2} c \\
-c^{3} & \sqrt{3} c^{2} d & d^{3} & \sqrt{3} c d^{2} \\
-\sqrt{3} a c^{2} & 2 a c d+b c^{2} & \sqrt{3} b d^{2} & 2 b c d+a d^{2}
\end{array}\right)
$$

Precomposition with $i: \pi_{1}(\Sigma) \rightarrow \mathrm{SL}(2, \mathbb{R})$ gives rise to an irreducible Fuchsian representation $\rho_{\text {irr }}: \pi_{1}(\Sigma) \stackrel{i}{\rightarrow} \mathrm{SL}(2, \mathbb{R}) \stackrel{\phi_{\text {irr }}}{\longrightarrow} \mathrm{Sp}(4, \mathbb{R})$.

ii) Diagonal Fuchsian representations

Let $\mathbb{R}^{4}=W_{1} \oplus W_{2}$, with $W_{i}=\operatorname{span}\left(e_{i}, e_{2+i}\right)$ be a symplectic splitting of $\mathbb{R}^{4}$ with respect to the symplectic basis $\left(e_{i}\right)_{i=1, \ldots, 4}$. This splitting gives rise to an embedding $\psi: \operatorname{SL}(2, \mathbb{R})^{2} \rightarrow$ 
$\operatorname{Sp}\left(W_{1}\right) \times \operatorname{Sp}\left(W_{2}\right) \subset \operatorname{Sp}(4, \mathbb{R})$ given by

$$
\psi\left(\left(\begin{array}{ll}
a & b \\
c & d
\end{array}\right),\left(\begin{array}{ll}
\alpha & \beta \\
\gamma & \delta
\end{array}\right)\right)=\left(\begin{array}{llll}
a & 0 & b & 0 \\
0 & \alpha & 0 & \beta \\
c & 0 & d & 0 \\
0 & \gamma & 0 & \delta
\end{array}\right)
$$

Precomposition with the diagonal embedding of $\mathrm{SL}(2, \mathbb{R}) \rightarrow \mathrm{SL}(2, \mathbb{R})^{2}$ gives rise to the diagonal embedding $\phi_{\Delta}: \mathrm{SL}(2, \mathbb{R}) \rightarrow \operatorname{Sp}(4, \mathbb{R})$, and precomposition with $i: \pi_{1}(\Sigma) \rightarrow \mathrm{SL}(2, \mathbb{R})$ gives now rise to a diagonal Fuchsian representation $\rho_{\Delta}: \pi_{1}(\Sigma) \stackrel{i}{\rightarrow} \mathrm{SL}(2, \mathbb{R}) \stackrel{\phi_{\Delta}}{\longrightarrow} \mathrm{Sp}(4, \mathbb{R})$.

iii) Twisted diagonal representations

For any maximal representation $\rho: \pi_{1}(\Sigma) \rightarrow \operatorname{Sp}(4, \mathbb{R})$ the centralizer $\rho\left(\pi_{1}(\Sigma)\right)$ is a subgroup of $\mathrm{O}(2)$. Considering now a representation $\Theta: \pi_{1}(\Sigma) \rightarrow \mathrm{O}(2)$, set

$$
\begin{aligned}
\rho_{\Theta}=i \otimes \Theta: \pi_{1}(\Sigma) & \rightarrow \operatorname{Sp}(4, \mathbb{R}) \\
\gamma & \mapsto \phi_{\Delta}(i(\gamma), \Theta(\gamma))
\end{aligned}
$$

Such a representation will be called twisted diagonal representation.

Remark 2.10. The representations in the families (i)-(iii) above are the so-called standard representations.

iv) Hybrid representations

The definition of hybrid representations involves a gluing construction for fundamental group representations over a connected sum of surfaces. Let $\Sigma=\Sigma_{l} \cup_{\gamma} \Sigma_{r}$ be a decomposition of the surface $\Sigma$ along a simple, closed, oriented, separating geodesic $\gamma$ into two subsurfaces $\Sigma_{l}$ and $\Sigma_{r}$. Pick $\rho_{\text {irr }}: \pi_{1}(\Sigma) \rightarrow \operatorname{SL}(2, \mathbb{R}) \stackrel{\phi_{i r r}}{\longrightarrow} \operatorname{Sp}(4, \mathbb{R})$ an irreducible Fuchsian representation and $\rho_{\Delta}: \pi_{1}(\Sigma) \rightarrow \mathrm{SL}(2, \mathbb{R}) \stackrel{\Delta}{\rightarrow} \mathrm{SL}(2, \mathbb{R})^{2} \rightarrow \mathrm{Sp}(4, \mathbb{R})$ a diagonal Fuchsian representation. One could amalgamate the restriction of the irreducible Fuchsian representation $\rho_{i r r}$ to $\Sigma_{l}$ with the restriction of the diagonal Fuchsian representation $\rho_{\Delta}$ to $\Sigma_{r}$, however the holonomies of those along $\gamma$ a priori do not agree. A deformation of $\rho_{\Delta}$ on $\pi_{1}(\Sigma)$ can be considered, such that the holonomies would agree along $\gamma$, thus allowing the amalgamation operation. This introduces new representations by gluing:

Definition 2.11. A hybrid representation is defined as the amalgamated representation

$$
\rho:=\left.\left.\rho_{l}\right|_{\pi_{1}\left(\Sigma_{l}\right)} * \rho_{r}\right|_{\pi_{1}\left(\Sigma_{r}\right)}: \pi_{1}(\Sigma) \simeq \pi_{1}\left(\Sigma_{l}\right) *\langle\gamma\rangle \pi_{1}\left(\Sigma_{r}\right) \rightarrow \operatorname{Sp}(4, \mathbb{R})
$$

If $\chi\left(\Sigma_{l}\right)=k$, then we call $\rho$ a $k$-hybrid representation.

The following important result was established in [20]:

Theorem 2.12 (O. Guichard, A. Wienhard [20]). Every maximal representation $\rho: \pi_{1}(\Sigma) \rightarrow$ $S p(4, \mathbb{R})$ can be deformed to a standard representation or a hybrid representation.

The subsurfaces $\Sigma_{l}$ and $\Sigma_{r}$ that we are considering here are surfaces with boundary. A notion of Toledo invariant can be also defined for representations over such surfaces and it thus makes sense to talk about maximal representations over surfaces with boundary as well; see [8] for a detailed definition. Moreover, the authors in [8] have established an additivity property for the Toledo invariant over a connected sum of surfaces. In particular: 
Proposition 2.13 ([8] Proposition 3.2). If $\Sigma=\Sigma_{1} \cup_{C} \Sigma_{2}$ is the connected sum of two subsurfaces $\Sigma_{i}$ along a separating loop $C$, then

$$
T_{\kappa}(\Sigma, \rho)=T_{\kappa}\left(\Sigma_{1}, \rho_{1}\right)+T_{\kappa}\left(\Sigma_{2}, \rho_{2}\right)
$$

where $\rho_{i}=\left.\rho\right|_{\pi_{1}\left(\Sigma_{i}\right)}, i=1,2$.

Note that this property implies that the amalgamated product of two maximal representations is again a maximal representation defined over the compact surface $\Sigma$.

\section{Parabolic $\operatorname{Sp}(4, \mathbb{R})$-Higgs bundles}

In this section we include the main definitions for parabolic $G$-Higgs bundles that we are going to be needing later on. We are primarily interested in the case $G=\operatorname{Sp}(4, \mathbb{R})$ and in describing the moduli space of maximal parabolic $\operatorname{Sp}(4, \mathbb{R})$-Higgs bundles. For the latter, a Milnor-Wood bound for an appropriate notion of Toledo invariant is necessary. A HitchinKobayashi correspondence provided in [3] for parabolic $G$-Higgs bundles points out that such holomorphic objects should correspond to representations of the fundamental group of a surface with boundary, with fixed monodromy around the boundary components. For a more detailed study of moduli of parabolic $\operatorname{Sp}(2 n, \mathbb{R})$-Higgs bundles, see [24].

3.1. Parabolic GL $(n, \mathbb{C})$-Higgs bundles. For further reference on the material covered in this section see [4], [6], or [17].

Definition 3.1. Let $X$ be a closed, connected, smooth Riemann surface of genus $g \geq 2$ and $D=\left\{x_{1}, \ldots, x_{s}\right\}$ a divisor of $s$-many distinct points on $X$; denote this pair by $(X, D)$. A parabolic vector bundle $E$ over $(X, D)$ is a holomorphic vector bundle $E \rightarrow X$ with parabolic structure at each $x \in D$ (weighted flag on each fiber $E_{x}$ ):

$$
\begin{gathered}
E_{x}=E_{x, 1} \supset E_{x, 2} \supset \ldots \supset E_{x, r(x)+1}=\{0\} \\
0 \leq \alpha_{1}(x)<\ldots<\alpha_{r(x)}(x)<1
\end{gathered}
$$

We usually write $(E, \alpha)$ to denote a vector bundle equipped with a parabolic structure determined by a system of weights $\alpha(x)=\left(\alpha_{1}(x), \ldots, \alpha_{n}(x)\right)$ at each $x \in D$. Moreover, set $k_{i}(x)=\operatorname{dim}\left(E_{x, i} / E_{x, i+1}\right)$ be the multiplicity of the weight $\alpha_{i}(x)$. We can also write the weights repeated according to their multiplicity as

$$
0 \leq \tilde{\alpha}_{1}(x) \leq \ldots \leq \tilde{\alpha}_{n}(x)<1
$$

where now $n=\operatorname{rkE}$. A weighted flag shall be called full, if $k_{i}(x)=1$ for every $i$ and $x \in D$.

Given a pair of parabolic vector bundles the basic constructions for a parabolic subbundle, direct sum, dual and tensor product have been described in [4] and [17]; we will be making frequent use of these constructions.

Definition 3.2. A holomorphic map $f: E \rightarrow E^{\prime}$ of parabolic vector bundles $(E, \alpha),\left(E^{\prime}, \alpha^{\prime}\right)$ is called parabolic if $\alpha_{i}(x)>\alpha^{\prime}{ }_{j}(x)$ implies $f\left(E_{x, i}\right) \subset E^{\prime}{ }_{x, j+1}$, for every $x \in D$.

Furthermore, we call such map strongly parabolic if $\alpha_{i}(x) \geq \alpha^{\prime}{ }_{j}(x)$ implies $f\left(E_{x, i}\right) \subset E^{\prime}{ }_{x, j+1}$ for every $x \in D$.

Definition 3.3. A notion of parabolic degree and parabolic slope of a vector bundle equipped with a parabolic structure can be defined as follows

$$
\operatorname{par} \operatorname{deg}(E)=\operatorname{deg} E+\sum_{x \in D} \sum_{i=1}^{r(x)} k_{i}(x) \alpha_{i}(x)
$$




$$
\operatorname{par} \mu(\mathrm{E})=\frac{\operatorname{pardeg}(E)}{r k(E)}
$$

Definition 3.4. A parabolic vector bundle will be called stable (resp. semistable), if for every non-trivial proper parabolic subbundle $F \leq E$, it is $\operatorname{par} \mu(F)<\operatorname{par} \mu(E)$, (resp. $\leq$ ).

Definition 3.5. Let $K$ be the canonical bundle over $X$ and $E$ a parabolic vector bundle. The bundle morphism $\Phi: E \rightarrow E \otimes K(D)$ will be called a parabolic Higgs field, if it preserves the parabolic structure at each point $x \in D$ :

$$
\left.\left.\Phi\right|_{x}\left(E_{x, i}\right) \subset E_{x, i} \otimes K(D)\right|_{x}
$$

In particular, we call $\Phi$ strongly parabolic, if

$$
\left.\left.\Phi\right|_{x}\left(E_{x, i}\right) \subset E_{x, i+1} \otimes K(D)\right|_{x}
$$

in other words, $\Phi$ is a meromorphic endomorphism valued 1-form with simple poles along the divisor $D$, whose residue at $x \in D$ is nilpotent with respect to the filtration. Note that the divisor $D$ is always considered to be a reduced divisor.

After these considerations we define parabolic Higgs bundles as follows:

Definition 3.6. Let $K$ be the canonical bundle over $X$ and $E$ be a parabolic vector bundle over $X$. A parabolic Higgs bundle over $(X, D)$ is given by a pair $(E, \Phi)$, where $\Phi: E \rightarrow$ $E \otimes K(D)$ is a strongly parabolic Higgs field.

Analogously to the non-parabolic case, we may define stability as follows:

Definition 3.7. A parabolic Higgs bundle will be called stable (resp. semistable), if for every $\Phi$-invariant parabolic subbundle $F \leq E$ it is $\operatorname{par} \mu(F)<\operatorname{par} \mu(E)$ (resp. $\leq$ ). Furthermore, it will be called polystable, if it is the direct sum of stable parabolic Higgs bundles of the same parabolic slope.

In [34], C. Simpson established a non-abelian Hodge correspondence for non-compact surfaces. A parabolic Higgs bundle is defined as a filtered regular Higgs bundle and for the construction of the correspondence in this case, it is necessary that the harmonic metric on the bundle has at most polynomial growth at the punctures in order to extend the holomorphic Higgs bundles across those points; these notions were introduced in [34] and the necessary growth condition of the hermitian metric, called tameness, is related to the algebraic stability of the filtered regular Higgs bundle. The topological objects that correspond to algebraically stable filtered regular Higgs bundles were called filtered local systems and the theorem of C. Simpson also provides a relation between the parabolic weights of a stable parabolic Higgs bundle and the holonomy of the associated flat connection around each puncture (cf. [34] for more information).

3.2. Parabolic $G$-Higgs bundles. In [3] the authors introduce parabolic $G$-Higgs bundles over a punctured Riemann surface for a non-compact real reductive Lie group $G$. This definition involves a choice for each puncture of an element in the Weyl alcove of a maximal compact subgroup $H \subset G$, handling both cases as if this element lies in the interior of the alcove or if it lies in a 'bad' wall of the alcove. Below we summarize the basic steps towards this definition.

Let $X$ be a compact, connected Riemann surface and let $\left\{x_{1}, \ldots, x_{s}\right\}$ be a finite set of different points on $X$ with $D=x_{1}+\ldots x_{s}$ be the corresponding effective divisor. Let now $H^{\mathbb{C}}$ be a reductive, complex Lie group. Fix a maximal compact subgroup $H \subset H^{\mathbb{C}}$, and a 
maximal torus $T \subset H$ with Lie algebra $\mathfrak{t}$. Denote $E\left(H^{\mathbb{C}}\right)=E \times_{H^{\mathbb{C}}} H^{\mathbb{C}} \rightarrow X$, the $H^{\mathbb{C}}$-fibration associated to $E$ via the adjoint representation of $H^{\mathbb{C}}$ on itself. Then

$$
E\left(H^{\mathbb{C}}\right)_{x}=\left\{\phi: E_{x} \rightarrow H^{\mathbb{C}} \mid \phi(e h)=h^{-1} \phi(e) h, \forall e \in E_{x}, h \in H^{\mathbb{C}}\right\}
$$

i.e. the fiber can be identified with the set of antiequivariant maps $\phi$.

Fix an alcove $\mathcal{A} \subset \mathfrak{t}$ of $H$ containing $0 \in \mathfrak{t}$ and for $\alpha_{i} \in \sqrt{-1} \overline{\mathcal{A}}$ let $P_{\alpha_{i}} \subset H^{\mathbb{C}}$ be the parabolic subgroup defined by the $\alpha_{i}$.

Definition 3.8. We define a parabolic structure of weight $\alpha_{i}$ on $E$ over a point $x_{i}$ as the choice of a subgroup $Q_{i} \subset E\left(H^{\mathbb{C}}\right)_{x_{i}}$ with the property that there exists a trivialization $e \in E_{x_{i}}$ for which $P_{\alpha_{i}}=\left\{\phi(e) \mid \phi \in Q_{i}\right\}$.

Given this, we now set the following:

Definition 3.9. Let $\alpha=\left(\alpha_{1}, \ldots, \alpha_{n}\right)$ be a collection of elements in $\sqrt{-1} \overline{\mathcal{A}}$. A parabolic bundle over $(X, D)$ of weight $\alpha$ is a holomorphic principal bundle with a choice for any $i$ of a parabolic structure of weight $\alpha_{i}$ over $x_{i}$.

Consider that the parabolic bundle $E$ comes equipped with a holomorphic structure $\bar{\partial}$ and consider a metric $h \in \Gamma(X \backslash D ; E / H)$ defined away from the divisor $D$.

Definition 3.10. The metric $h$ is called an $\alpha$-adapted metric if for any parabolic point $x_{i}$ the following holds: Let $e_{i} \in E_{x_{i}}$ be an element belonging to the $P_{\alpha_{i}}$ orbit specified by the parabolic structure. Choose local holomorphic coordinate $z$ and extend the trivialization $e_{i}$ into a holomorphic trivialization of $E$ near $x_{i}$. Then we can write near $x_{i}$

$$
h=\left(|z|^{-\alpha_{i}} e^{c}\right)^{2}
$$

with $\operatorname{Ad}\left(|z|^{-\alpha_{i}}\right) c=\mathrm{o}(\log |z|), \operatorname{Ad}\left(|z|^{-\alpha_{i}}\right) d c \in L^{2}$ and $\operatorname{Ad}\left(|z|^{-\alpha_{i}}\right) F_{h} \in L^{1}$.

For a real reductive Lie group $G$ with a maximal compact subgroup $H$, let $\mathfrak{g}=\mathfrak{h} \oplus \mathfrak{m}$ the Cartan decomposition of its Lie algebra into its \pm 1-eigenspaces, where $\mathfrak{h}=\operatorname{Lie}(H)$ and let $E\left(\mathfrak{m}^{\mathbb{C}}\right)$ be the bundle associated to $E$ via the isotropy representation. Choose a trivialization $e \in E$ near the point $x_{i}$, such that near $x_{i}$ the parabolic weight lies in $\alpha_{i} \in \sqrt{-1} \overline{\mathcal{A}}$. In the trivialization $e$, we can decompose the bundle $E\left(\mathfrak{m}^{\mathbb{C}}\right)$ under the eigenvalues of ad $\left(\alpha_{i}\right)$ acting on $\mathfrak{m}^{\mathbb{C}}$ as

$$
E\left(\mathfrak{m}^{\mathbb{C}}\right)=\bigoplus_{\mu} \mathfrak{m}_{\mu}^{\mathbb{C}}
$$

Decompose accordingly a section $\varphi$ of $E\left(\mathfrak{m}^{\mathbb{C}}\right)$ as $\varphi=\sum \varphi_{\mu}$. After these preliminaries we set the following:

Definition 3.11. The sheaf $P E\left(\mathfrak{m}^{\mathbb{C}}\right)$ of parabolic sections (resp. the sheaf $N E\left(\mathfrak{m}^{\mathbb{C}}\right)$ of strictly parabolic sections) of $E\left(\mathfrak{m}^{\mathbb{C}}\right)$ is consisted of meromorphic sections $\varphi$ of the bundle $E\left(\mathfrak{m}^{\mathbb{C}}\right)$ with singularities at the points $x_{i}$, with $\varphi_{\mu}$ having order

$$
v\left(\varphi_{\mu}\right) \geq-\lfloor-\mu\rfloor \quad\left(\text { resp. } v\left(\varphi_{\mu}\right)>-\lfloor-\mu\rfloor\right)
$$

This means that if $a-1<\mu \leq a$ (resp. $a-1 \leq \mu<a$ ) for some integer $a$, then $\varphi_{\mu}=\mathrm{O}\left(z^{a}\right)$.

We finally have the definition of a parabolic $G$-Higgs bundle as follows:

Definition 3.12. We define a parabolic G-Higgs bundle over a Riemann surface with a divisor $(X, D)$ to be a pair $(E, \varphi)$, where: 
(1) $E$ is a parabolic principal $H^{\mathbb{C}}$-bundle over $(X, D)$, and

(2) $\varphi$ is a holomorphic section of $P E\left(\mathfrak{m}^{\mathbb{C}}\right) \otimes K(D)$.

The pair $(E, \varphi)$ will be called a strictly parabolic G-Higgs bundle if in addition the Higgs field $\varphi$ is a section of $N E\left(\mathfrak{m}^{\mathbb{C}}\right) \otimes K(D)$.

For a parabolic principal bundle $E$ over $(X, D)$ with weights $\alpha$, a notion of parabolic degree was defined in [3] as the sum of two terms, one global and independent of the parabolic structure, and one local and depending on the parabolic structure. Before we state this definition, recall that the degree of a (non-parabolic) bundle can be defined using Chern-Weil theory as follows:

Fix a standard parabolic subgroup $P \subset H^{\mathbb{C}}$, an antidominant character $\chi: \mathfrak{p} \rightarrow \mathbb{C}$ and a holomorphic reduction $\sigma$ of the structure group of $E$ from $H^{\mathbb{C}}$ to $P$, with $E_{\sigma}$ denoting the $P$-principal bundle corresponding to this reduction $\sigma$. Then, the degree of $E$ is given by

$$
\operatorname{deg}(E)(\sigma, \chi):=\frac{\sqrt{-1}}{2 \pi} \int_{X} \chi_{*}\left(F_{A}\right)
$$

where $F_{A}$ is the curvature of any $P$-connection $A$ on $E_{\sigma}$.

Now, let $Q_{i} \subset E\left(H^{\mathbb{C}}\right)_{x_{i}}$ the parabolic subgroups in the definition of the parabolic structure. At each point in the divisor $D$, there are two parabolic subgroups equipped with an antidominant character, one coming from the parabolic structure $\left(Q_{i}, \alpha_{i}\right)$ and one coming from the reduction $\left(E_{\sigma}(P)_{x_{i}}, \chi\right)$. A relative degree for such a pair of parabolic subgroups $(\mathcal{Q}, \mathcal{P})$ is then defined:

$$
\operatorname{deg}((\mathcal{Q}, \sigma),(\mathcal{P}, s))=\cos \angle_{\text {Tits }}(\eta(\sigma), \eta(s))
$$

where $\angle_{\text {Tits }}$ is the Tits distance on $\partial_{\infty} \mathcal{X}$ for $\mathcal{X}=H \backslash G$ a symmetric space of non-compact type, and $\eta(s)=\lim _{t \rightarrow \infty} * e^{t s} \in \partial_{\infty} \Sigma$ for $s$ in an $H$-orbit $\mathcal{O}_{H} \subset \mathfrak{m}$. The parabolic degree is now given by the sum of the two terms described previously:

$$
\operatorname{pardeg}_{\alpha}(E)(\sigma, \chi):=\operatorname{deg}(E)(\sigma, \chi)-\sum_{i} \operatorname{deg}\left(\left(Q_{i}, \alpha_{i}\right),\left(E_{\sigma}(P)_{x_{i}}, \chi\right)\right)
$$

The definition of polystability is next given with respect to an element $c \in \sqrt{-1}$ l for $\mathfrak{l}=\operatorname{Lie}(Z(H))$ :

Definition 3.13. Let $(E, \Phi)$ be a parabolic $G$-Higgs bundle over $(X, D)$. Then $(E, \Phi)$ will be called polystable if for every $s \in \sqrt{-1} \mathfrak{h}$ and any holomorphic reduction $\sigma$ of the structure group of $E$ to $P_{s}$, such that $\left.\Phi\right|_{X \backslash D} \in H^{0}\left(X \backslash D, E_{\sigma}\left(\mathfrak{m}_{s}\right) \otimes K\right)$ it is

$$
\operatorname{par} \operatorname{deg}(E)\left(\sigma, \chi_{s}\right)-\langle c, s\rangle \geq 0
$$

The following theorem proven in [3] establishes a Hitchin-Kobayashi correspondence for parabolic $G$-Higgs bundles.

Theorem 3.14 (O. Biquard, O. García-Prada, I. Mundet i Riera [3]). Let (E, $\Phi)$ be a parabolic $G$-Higgs bundle equipped with an adapted initial metric $h_{0}$. Suppose that par $\operatorname{deg}(E)=\chi(c)$ for all characters of $\mathfrak{g}$. Then $(E, \Phi)$ admits an Hermite-Einstein metric $h$, quasi-isometric to $h_{0}$, if and only if $(E, \Phi)$ is polystable. 
3.3. Parabolic $\mathbf{S p}(\mathbf{4}, \mathbb{R})$-Higgs bundles. The general parabolic $G$-Higgs bundle definitions of $\S 3.2$ can be now restricted to the case when the group $G=\operatorname{Sp}(4, \mathbb{R})$ to the following (see [24] for a more detailed exposition):

Definition 3.15. Let $X$ be a compact Riemann surface of genus $g$ and let the divisor $D:=$ $\left\{x_{1}, \ldots, x_{s}\right\}$ of $s$-many distinct points on $X$, assuming that $2 g-2+s>0$. Fix a line bundle $\iota=\mathcal{O}_{X}(D)$ over the divisor $D$. A parabolic $S p(4, \mathbb{R})$-Higgs bundle is defined as a triple $(V, \beta, \gamma)$, where:

- $V$ is a rank 2 bundle on $X$, equipped with a parabolic structure given by a flag $V_{x} \supset L_{x} \supset 0$ and weights $0 \leq \alpha_{1}(x)<\alpha_{2}(x)<1$ for every $x \in D$, and

- $\beta: V^{\vee} \rightarrow V \otimes K \otimes \iota$ and $\gamma: V \rightarrow V^{\vee} \otimes K \otimes \iota$ are strongly parabolic symmetric homomorphisms,

and where $V^{\vee}:=(V \otimes \iota)^{*}$ denotes the parabolic dual of the parabolic bundle $V$.

A notion of parabolic Toledo invariant can still be considered:

Definition 3.16. The parabolic Toledo invariant of a parabolic Sp $(4, \mathbb{R})$-Higgs bundle is defined as the rational number

$$
\tau=\operatorname{par} \operatorname{deg}(V)
$$

Moreover, we get a Milnor-Wood type inequality for this topological invariant:

Proposition 3.17. Let $(E, \Phi)$ be a semistable parabolic $S p(4, \mathbb{R})$-Higgs bundle. Then

$$
|\tau| \leq 2 g-2+s
$$

where $s$ is the number of punctures on the surface $X$.

Proof. Adaptation in the parabolic case of the proof of Proposition 3.1 from [19].

Definition 3.18. The parabolic Sp $(4, \mathbb{R})$-Higgs bundles with parabolic Toledo invariant $\tau=$ $2 g-2+s$ will be called maximal and we will denote the components containing such triples by

$$
\mathcal{M}_{\text {par }}^{\max }:=\mathcal{M}_{\text {par }}^{2 g-2+s}
$$

\section{Producing approximate solutions By Gluing}

In this section we develop a gluing construction for solutions to the $\operatorname{Sp}(4, \mathbb{R})$-Hitchin equations over a connected sum of Riemann surfaces to produce an approximate solution of the equations. The necessary condition in order to combine the initial parabolic data over the connected sum operation is that this data is identified over annuli around the points in the divisors of the Riemann surfaces. Aiming to provide new model Higgs bundles in the exceptional components of $\mathcal{M}^{\mathrm{max}}$, we consider parabolic data which around the punctures are $a$ priori not identified, but we will rather seek for deformations of those into model solutions of the Hitchin equations which will allow us to combine data over the complex connected sum. This deformation argument is coming from deformations of $\mathrm{SL}(2, \mathbb{R})$-solutions to the Hitchin equations over a punctured surface and subsequently we extend this for $\operatorname{Sp}(4, \mathbb{R})$-pairs using appropriate embeddings $\phi: \mathrm{SL}(2, \mathbb{R}) \hookrightarrow \mathrm{Sp}(4, \mathbb{R})$. Therefore, our gluing construction involves parabolic $\operatorname{Sp}(4, \mathbb{R})$-pairs which arise from $\mathrm{SL}(2, \mathbb{R})$-pairs via extensions by such embeddings. 
4.1. The local model. We describe the local $\mathrm{SL}(2, \mathbb{R})$-model solutions to the Hitchin equations which are going to serve as a guide for the gluing construction of the parabolic stable $\operatorname{Sp}(4, \mathbb{R})$-Higgs bundles. The description of these models is obtained by studying the behavior of the harmonic map between a surface $X$ with a given complex structure and the surface $X$ with the corresponding Riemannian metric of constant curvature -4 , under degeneration of the domain Riemann surface $X$ to a noded surface; cf. [36], [39] for further details.

Let $\mathcal{C}=\left(S^{1}\right)_{x} \times[1, \infty)_{y}$ denote the half-infinite cylinder, endowed with the complex coordinate $z=x+i y$ and flat Riemannian metric $g_{\mathcal{C}}=|d z|^{2}=d x^{2}+d y^{2}$. For parameter $s>0$ let

$$
N_{s}=\left[s^{-1} \csc ^{-1}\left(s^{-1}\right), \frac{\pi}{s}-s^{-1} \csc ^{-1}\left(s^{-1}\right)\right]_{u} \times\left(S^{1}\right)_{v}
$$

be the finite cylinder with complex coordinate $w=u+i v$, and carrying the hyperbolic metric $g_{s}=s^{2} \csc ^{2}(s u)|d w|^{2}$. It is shown in [39] that the one parameter family $w_{s}:\left(\mathcal{C},|d z|^{2}\right) \rightarrow$ $\left(N_{s}, g_{s}\right)$ with $w_{s}=u_{s}+i v_{s}$ and where $v_{s}(x, y)=x, u_{s}(x, y)=\frac{1}{s} \sin ^{-1}\left(\frac{1-B_{s}(y)}{1+B_{s}(y)}\right)$, for $B_{s}(y)=$ $\frac{1-s}{1+s} e^{2 s(1-y)}$, serves as a model for harmonic maps with domain a noded Riemann surface and target a smooth Riemann surface containing a long hyperbolic neck with central geodesic of length $2 \pi s$.

For a stable $\operatorname{SL}(2, \mathbb{R})$-Higgs bundle $(E, \Phi)$ on $X$ with $E=L \oplus L^{-1}$ for $L$ a holomorphic square root of the canonical line bundle over $X$, endowed with an auxiliary hermitian metric $h_{0}$, and $\Phi=\left(\begin{array}{ll}0 & q \\ 1 & 0\end{array}\right) \in H^{0}(X, \mathfrak{s l}(E))$ for $q$ a holomorphic quadratic differential, there is an induced hermitian metric $H_{0}=h_{0} \oplus h_{0}^{-1}$ on $E$ and $A=A_{L} \oplus A_{L}^{-1}$ the associated Chern connection with respect to $h$. The stability condition implies that there exists a complex gauge transformation $g$ unique up to unitary gauge transformations, such that $\left(A_{1, s}, \Phi_{1, s}\right):=g^{*}(A, \Phi)$ is a solution to the Hitchin equations. Choosing a local holomorphic trivialization on $E$ and assuming that with respect to it the auxiliary hermitian metric $h_{0}$ is the standard hermitian metric on $\mathbb{C}^{2}$, the corresponding hermitian metric for this solution on the bundle $E=L \oplus L^{-1}$ is globally well-defined with respect to the holomorphic splitting of $E$ into line bundles. Calculations worked out in [36] imply that in particular $H_{1, s}=\left(\begin{array}{cc}h_{1, s} & 0 \\ 0 & h_{1, s}^{-1}\end{array}\right)$, for

$$
h_{1, s}=\frac{2}{s}\left(\frac{1-B_{s}^{1 / 2}}{1+B_{s}^{1 / 2}}\right)
$$

the hermitian metric on $L$ and $g_{s}$ with $g_{s}^{2}=H_{1, s}^{-1}$ is the complex gauge transformation giving rise to an exact solution $\left(A_{1, s}, \Phi_{1, s}\right)$ of the self-duality equations.

Moreover, after the change in coordinates

$$
\zeta=e^{i z}, i d z=\frac{d \zeta}{\zeta}
$$

which describes the conformal mapping of the cylinder $\mathcal{C}$ to the punctured unit disk, one sees that

$$
A_{1, s}=O\left(|\zeta|^{s}\right)\left(\begin{array}{cc}
1 & 0 \\
0 & -1
\end{array}\right)\left(\frac{d \zeta}{\zeta}-\frac{d \bar{\zeta}}{\bar{\zeta}}\right), \Phi_{1, s}=\left(1+O\left(|\zeta|^{s}\right)\right)\left(\begin{array}{cc}
0 & \frac{s}{2} \\
\frac{s}{2} & 0
\end{array}\right) \frac{d \zeta}{i \zeta}
$$


Therefore, after a unitary change of frame, the Higgs field $\Phi_{1, s}$ is asymptotic to the model Higgs field $\Phi_{s}^{\text {mod }}=\left(\begin{array}{cc}\frac{s}{2} & 0 \\ 0 & -\frac{s}{2}\end{array}\right) \frac{d \zeta}{i \zeta}$, while the connection $A_{1, s}$ is asymptotic to the trivial flat connection.

In conclusion, the model solution to the $\mathrm{SL}(2, \mathbb{R})$-Hitchin equations we will be considering is described by

$$
A^{\bmod }=0, \Phi^{\bmod }=\left(\begin{array}{cc}
C & 0 \\
0 & -C
\end{array}\right) \frac{d z}{z}
$$

over a punctured disk with $z$-coordinates around the puncture with the condition that $C \in \mathbb{R}$ with $C \neq 0$, and that the meromorphic quadratic differential $q:=\operatorname{det} \Phi$ mod has at least one simple zero. That this is indeed the generic case, is discussed in [25].

4.2. Weighted Sobolev spaces. In order to develop the necessary analytic arguments for the gluing construction later on, we need to define Sobolev spaces. Let $X$ be a compact Riemann surface and $D:=\left\{p_{1}, \ldots, p_{s}\right\}$ be a collection of $s$-many distinct points on $X$. Moreover, let $(E, h)$ be a hermitian vector bundle on $E$. Choose an initial pair $\left(A^{\bmod }, \Phi^{\bmod }\right)$ on $E$, such that in some unitary trivialization of $E$ around each point $p \in D$, the pair coincides with the local model from $\S 4$.1. Of course, on the interior of each region $X \backslash\{p\}$ the pair $\left(A^{\text {mod }}, \Phi^{\text {mod }}\right)$ need not satisfy the Hitchin equations.

For fixed local coordinates $z$ around each point $p \in D$, such that $z(p)=0$, define $r$ to be a positive function which coincides with $|z|$ around the puncture. Using the singular measure $r^{-1} d r d \theta$ and a fixed weight $\delta>0$ define weighted $L^{2}$-Sobolev spaces:

$$
L_{\delta}^{2}=\left\{f \in L^{2}(r d r d \theta) \mid \frac{f}{r^{\delta+1}} \in L^{2}\right\}
$$

and

$$
H_{\delta}^{k}=\left\{u, \nabla^{j} u \in L_{\delta}^{2}(r d r), 0 \leq j \leq k\right\} .
$$

The Sobolev space with $k$-derivatives in $L^{2}$ is defined as:

$$
L_{\delta}^{k, 2}=\left\{f, \frac{\nabla^{j} f}{r^{k-j}} \in L_{\delta}^{2}(r d r d \theta), 0 \leq j \leq k\right\}
$$

where $\nabla$ is the covariant derivative associated to a fixed background unitary connection on $E$. We are interested in deformations of $A$ and $\Phi$ such that the curvature of the connection $D=A+\Phi+\Phi^{*}$ remains $O\left(r^{-2+\delta}\right)$, that is, slightly better than $L^{1}$. We can then define global Sobolev spaces on $X$ as the spaces of admissible deformations of the model unitary connection and the model Higgs field $\left(A^{\bmod }, \Phi^{\bmod }\right)$ as:

$$
\mathcal{A}=\left\{A^{\bmod }+\alpha \mid \alpha \in H_{-2+\delta}^{1,2}\left(\Omega^{1} \otimes \mathfrak{s u}(E)\right)\right\}
$$

and

$$
\mathcal{B}=\left\{\Phi^{\bmod }+\varphi \mid \varphi \in H_{-2+\delta}^{1,2}\left(\Omega^{1,0} \otimes \operatorname{End}(E)\right)\right\}
$$

The space of unitary gauge transformations

$$
\mathcal{G}=\left\{g \in \mathrm{U}(E), g^{-1} d g \in L_{-2+\delta}^{1,2}\right\}
$$

acts on $\mathcal{A}$ and $\mathcal{B}$ as follows

$$
g^{*}(A, \Phi)=\left(g^{-1} A g+g^{-1} d g, g^{-1} \Phi g\right)
$$

for a pair $(A, \Phi) \in \mathcal{A} \times \mathcal{B}$. 
These considerations allow us to introduce the moduli space of solutions which are close to the model solution over a punctured Riemann surface $X^{\times}:=X-D$ for some fixed parameter $C \in \mathbb{R}:$

$$
\mathcal{M}\left(X^{\times}\right)=\frac{\{(A, \Phi) \in \mathcal{A} \times \mathcal{B} \mid(A, \Phi) \text { satisfies the Hitchin equations }\}}{\mathcal{G}}
$$

This moduli space was explicitly constructed by H. Konno in [23] as a hyperkähler quotient.

4.3. Approximate solutions of the $\mathbf{S L}(2, \mathbb{R})$-Hitchin equations. In $\S 4.2$ we saw that a point in the moduli space $\mathcal{M}\left(X^{\times}\right)$differs from a model pair $\left(A^{\text {mod }}, \Phi^{\text {mod }}\right)$ by some element in $H_{-2+\delta}^{1}$. The following result by $\mathrm{O}$. Biquard and $\mathrm{P}$. Boalch shows that $(A, \Phi)$ is asymptotically close to the model in a much stronger sense:

Lemma 4.1. Lemma 5.3 in [2]. For each point $p \in D$ let $\left(A_{p}^{\bmod }, \Phi_{p}^{\bmod }\right)$ be a model pair as was defined in $\S 4.1$. If $(A, \Phi) \in \mathcal{M}\left(X^{\times}\right)$, then there exists a unitary gauge transformation $g \in \mathcal{G}$ such that in a neighborhood of each point $p \in D$ it is

$$
g^{*}(A, \Phi)=\left(A_{p}^{\bmod }, \Phi_{p}^{\bmod }\right)+O\left(r^{-1+\delta}\right)
$$

for a positive constant $\delta$.

The decay described in this lemma can be further improved by showing that in a suitable complex gauge transformation the point $(A, \Phi)$ coincides precisely with the model near each puncture in $D$. With respect to the singular measure $r^{-1} d r d \vartheta$ on $\mathbb{C}$, we first introduce the Hilbert spaces

$$
\begin{gathered}
L_{-1+\delta}^{2}\left(r^{-1} d r d \vartheta\right)=\left\{u \in L^{2}(\mathbb{D}) \mid r^{-\delta} u \in L^{2}\left(r^{-1} d r d \vartheta\right)\right\} \\
H_{-1+\delta}^{k}\left(r^{-1} d r d \vartheta\right)=\left\{u \in L^{2}(\mathbb{D}) \mid(r \partial r)^{j} \partial_{\vartheta}^{l} u \in L_{-1+\delta}^{2}\left(r^{-1} d r d \vartheta\right), 0 \leq j+l \leq k\right\}
\end{gathered}
$$

for $\mathbb{D}=\{z \in \mathbb{C}|0<| z \mid<1\}$ the punctured unit disk. We then have the following result by J. Swoboda:

Lemma 4.2. Lemma 3.2 in [36]. Let $(A, \Phi) \in \mathcal{M}\left(X^{\times}\right)$and let $\delta$ be the constant provided by Lemma 4.1. Fix another constant $0<\delta^{\prime}<\min \left\{\frac{1}{2}, \delta\right\}$. Then there is a complex gauge transformation $g=\exp (\gamma) \in \mathcal{G}^{c}$ with $\gamma \in H_{-1+\delta^{\prime}}^{2}\left(r^{-1} d r d \vartheta\right)$, such that $g^{*}(A, \Phi)$ coincides with $\left(A_{p}^{\text {mod }}, \Phi_{p}^{\text {mod }}\right)$ in a sufficiently small neighborhood of the point $p$, for each $p \in D$.

We shall now use this complex gauge transformation as well as a smooth cut-off function to obtain an approximate solution to the $\mathrm{SL}(2, \mathbb{R})$-Hitchin equations. For the fixed local coordinates $z$ around each puncture $p$ and the positive function $r$ coinciding with $|z|$ around the puncture, fix a constant $0<R<1$ and choose a smooth cut-off function $\chi_{R}:[0, \infty) \rightarrow[0,1]$ with supp $\chi \subseteq[0, R]$ and $\chi_{R}(r)=1$ for $r \leq \frac{3 R}{4}$. We impose the further requirement on the growth rate of this cut-off function:

$$
\left|r \partial_{r} \chi_{R}\right|+\left|(r \partial r)^{2} \chi_{R}\right| \leq C
$$

for some constant $C$ not depending on $R$.

The map $x \mapsto \chi_{R}(r(x)): X^{\times} \rightarrow \mathbb{R}$ gives rise to a smooth cut-off function on the punctured surface $X^{\times}$which by a slight abuse of notation we shall still denote by $\chi_{R}$. We may use this function $\chi_{R}$ to glue the two pairs $(A, \Phi)$ and $\left(A_{p}^{\bmod }, \Phi_{p}^{\bmod }\right)$ into an approximate solution

$$
\left(A_{R}^{a p p}, \Phi_{R}^{a p p}\right):=\exp \left(\chi_{R} \gamma\right)^{*}(A, \Phi) .
$$


The pair $\left(A_{R}^{a p p}, \Phi_{R}^{a p p}\right)$ is a smooth pair and is by construction an exact solution of the Hitchin equations away from each punctured neighborhood $\mathcal{U}_{p}$, while it coincides with the model pair $\left(A_{p}^{\text {mod }}, \Phi_{p}^{\text {mod }}\right)$ near each puncture. More precisely, we have:

$$
\left(A_{R}^{a p p}, \Phi_{R}^{a p p}\right)=\left\{\begin{array}{c}
(A, \Phi), \text { over } X \backslash \bigcup_{p \in D}\left\{z \in \mathcal{U}_{p}\left|\frac{3 R}{4} \leq\right| z \mid \leq R\right\} \\
\left(A_{p}^{\text {mod }}, \Phi_{p}^{\text {mod }}\right), \text { over }\left\{z \in \mathcal{U}_{p}|0<| z \mid \leq \frac{3 R}{4}\right\}, \text { for each } p \in D
\end{array}\right.
$$
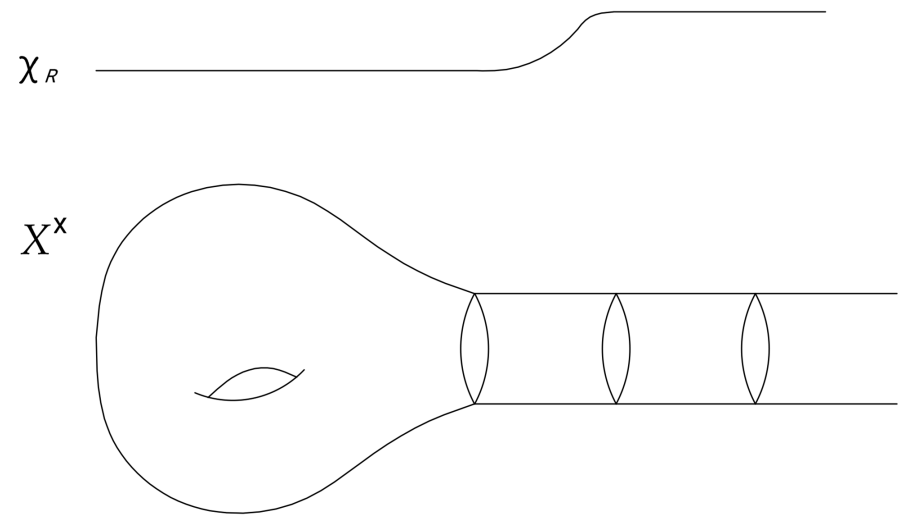

Figure 1. Constructing an approximate solution over the punctured surface $X^{\times}$.

Since $\left(A_{R}^{a p p}, \Phi_{R}^{a p p}\right)$ is complex gauge equivalent to an exact solution $(A, \Phi)$ of the Hitchin equations, it does still satisfy the second equation, in other words, it holds that $\bar{\partial}_{A_{R}^{a p p}} \Phi_{R}^{a p p}=0$. Indeed, for $\tilde{g}:=\exp \left(\chi_{R} \gamma\right)$, we defined $\left(A_{R}^{a p p}, \Phi_{R}^{a p p}\right)=\tilde{g}^{*}(A, \Phi)=\left(\tilde{g}^{-1} A \tilde{g}+\tilde{g}^{-1} d \tilde{g}, \tilde{g}^{-1} \Phi \tilde{g}\right)$ and $(A, \Phi)$ is an exact solution, thus in particular

$$
0=\bar{\partial}_{A} \Phi=\bar{\partial} \Phi+\left[A^{0,1} \wedge \Phi\right]
$$

We may now check

$$
\begin{aligned}
\bar{\partial}_{A_{R}^{a p p}} \Phi_{R}^{a p p} & =\bar{\partial} \Phi_{R}^{a p p}+\left[\left(A_{R}^{a p p}\right)^{0,1} \wedge \Phi_{R}^{a p p}\right] \\
& =\bar{\partial}\left(\tilde{g}^{-1} \Phi \tilde{g}\right)+\left[\left(\tilde{g}^{-1} A^{0,1} \tilde{g}+\tilde{g}^{-1} \bar{\partial} \tilde{g}\right) \wedge \tilde{g}^{-1} \Phi \tilde{g}\right] \\
& =\bar{\partial}\left(\tilde{g}^{-1} \Phi \tilde{g}\right)+\tilde{g}^{-1}\left[A^{0,1} \wedge \Phi\right] \tilde{g}+\tilde{g}^{-1}(\bar{\partial} \tilde{g}) \tilde{g}^{-1} \Phi \tilde{g}-\tilde{g}^{-1} \Phi \bar{\partial} \tilde{g} \\
& =\bar{\partial}\left(\tilde{g}^{-1} \Phi \tilde{g}\right)+\tilde{g}^{-1}(-\bar{\partial} \Phi) \tilde{g}+\tilde{g}^{-1}(\bar{\partial} \tilde{g}) \tilde{g}^{-1} \Phi \tilde{g}-\tilde{g}^{-1} \Phi \bar{\partial} \tilde{g} \\
& =\bar{\partial}\left(\tilde{g}^{-1}\right) \Phi \tilde{g}+\tilde{g}^{-1}(\bar{\partial} \tilde{g}) \tilde{g}^{-1} \Phi \tilde{g}=0,
\end{aligned}
$$

using the identity $(\bar{\partial} \tilde{g}) \tilde{g}^{-1}+\tilde{g} \bar{\partial}\left(\tilde{g}^{-1}\right)=0$.

Moreover, Lemma 4.2 as well as the Assumption (4.1) we made on the growth rate of the bump function $\chi_{R}$ provide us with a good estimate of the error up to which $\left(A_{R}^{a p p}, \Phi_{R}^{a p p}\right)$ satisfies the first equation:

Lemma 4.3. Let $\delta^{\prime}>0$ be as in Lemma 4.2 and fix some further constant $0<\delta^{\prime \prime}<\delta^{\prime}$. The approximate solution $\left(A_{R}^{\text {app }}, \Phi_{R}^{\text {app }}\right)$ to the parameter $0<R<1$ satisfies

$$
\left\|* F_{A_{R}^{a p p}}^{\perp}+*\left[\Phi_{R}^{a p p} \wedge\left(\Phi_{R}^{a p p}\right)^{*}\right]\right\|_{C^{0}\left(X^{\times}\right)} \leq C R^{\delta^{\prime \prime}}
$$


for some constant $C=C\left(\delta^{\prime}, \delta^{\prime \prime}\right)$ which does not depend on $R$.

Proof. See [36] Lemma 3.5.

4.4. Extending $\mathbf{S L}(\mathbf{2}, \mathbb{C})$-pairs into $\mathbf{S p}(4, \mathbb{C})$. We will now use the approximate solutions from $\S 4.3$ in order to obtain an approximate solution by gluing parabolic Higgs bundles. Let $X_{1}$ be a closed Riemann surface of genus $g_{1}$ and $D_{1}=\left\{p_{1}, \ldots, p_{s}\right\}$ a collection of distinct points on $X_{1}$. Let $\left(E_{1}, \Phi_{1}\right) \rightarrow X_{1}$ be a parabolic stable $\mathrm{SL}(2, \mathbb{R})$-Higgs bundle. Then there exists an adapted Hermitian metric $h_{1}$, such that $\left(D_{h_{1}}, \Phi_{1}\right)$ is a solution to the equations, with $D_{h_{1}}=\nabla\left(\bar{\partial}_{1}, h_{1}\right)$ the associated Chern connection.

Let $g_{1}=\exp \left(\gamma_{1}\right)$ be the complex gauge transformation from $\S 4.3$, such that $g_{1}^{*}\left(D_{h_{1}}, \Phi_{1}\right)$ is asymptotically close to a model solution $\left(A_{1, p}^{\bmod }, \Phi_{1, p}^{\bmod }\right)$ near the puncture $p$, for each $p \in D_{1}$. Choose a trivialization $\tau$ over a neighborhood $\mathcal{U}_{p} \subset X_{1}$ so that $\left(D_{h_{1}}\right)^{\tau}$ denotes the connection matrix and let $\chi_{1}$ be a smooth bump function on $\mathcal{U}_{p}$ with the assumptions made in $\S 4.3$, so that we may define $\tilde{g}_{1}=\exp \left(\chi_{1} \gamma_{1}\right)$ and take the approximate solution over $X_{1}$

$$
\left(A_{1}^{a p p}, \Phi_{1}^{a p p}\right)=\tilde{g}_{1}^{*}\left(D_{h_{1}}, \Phi_{1}\right)=\left\{\begin{array}{l}
\left(D_{h_{1}}, \Phi_{1}\right), \text { away from the points in the divisor } D_{1} \\
\left(A_{1, p}^{\text {mod }}, \Phi_{1, p}^{\text {mod }}\right), \text { near the point } p, \text { for each } p \in D_{1}
\end{array}\right.
$$

The connection $A_{1}^{a p p}$ is given, in that same trivialization, by the connection matrix $\chi_{1}\left(D_{h_{1}}\right)^{\tau}$. The fact that $\tilde{g}_{1}$ is a complex gauge transformation may cause the holonomy over the bump region not to be real, so a priori we are considering this pair as $S L(2, \mathbb{C})$-data.

We wish to obtain an approximate $\operatorname{Sp}(4, \mathbb{C})$-pair by extending the $\operatorname{SL}(2, \mathbb{C})$-data via an embedding

$$
\phi: \mathrm{SL}(2, \mathbb{R}) \hookrightarrow \mathrm{Sp}(4, \mathbb{R})
$$

and its extension $\phi: \operatorname{SL}(2, \mathbb{C}) \hookrightarrow \operatorname{Sp}(4, \mathbb{C})$. For the Cartan decompositions

$$
\begin{aligned}
\mathfrak{s l}(2, \mathbb{R}) & =\mathfrak{s o}(2) \oplus \mathfrak{m}(\operatorname{SL}(2, \mathbb{R})) \\
\mathfrak{s p}(4, \mathbb{R}) & =\mathfrak{u}(2) \oplus \mathfrak{m}(\operatorname{Sp}(4, \mathbb{R}))
\end{aligned}
$$

their complexifications respectively read

$$
\begin{aligned}
\mathfrak{s l}(2, \mathbb{C}) & =\mathfrak{s o}(2, \mathbb{C}) \oplus \mathfrak{m}^{\mathbb{C}}(\operatorname{SL}(2, \mathbb{R})) \\
\mathfrak{s p}(4, \mathbb{C}) & =\mathfrak{g l}(2, \mathbb{C}) \oplus \mathfrak{m}^{\mathbb{C}}(\operatorname{Sp}(4, \mathbb{R}))
\end{aligned}
$$

Assume now that copies of a maximal compact subgroup of $\operatorname{SL}(2, \mathbb{R})$ are mapped via $\phi$ into copies of a maximal compact subgroup of $\mathrm{Sp}(4, \mathbb{R})$. Then, since $\mathrm{SO}(2)^{\mathbb{C}}=\mathrm{SO}(2, \mathbb{C})$ and $\mathrm{U}(2)^{\mathbb{C}}=\mathrm{GL}(2, \mathbb{C})$, the embedding $\phi$ describes an embedding $\mathrm{SO}(2, \mathbb{C}) \hookrightarrow \mathrm{GL}(2, \mathbb{C})$ and so we may use its infinitesimal deformation $\phi_{*}: \mathfrak{s l}(2, \mathbb{C}) \rightarrow \mathfrak{s p}(4, \mathbb{C})$ to extend $\mathrm{SL}(2, \mathbb{C})$-data to $\operatorname{Sp}(4, \mathbb{C})$-data (see [29], $\S 5.4,5.5$ for details).

We shall denote the $\operatorname{Sp}(4, \mathbb{C})$-pair obtained by extension through $\phi$ by $\left(A_{l}, \Phi_{l}\right)$, with the curvature of the connection denoted by

$$
F_{A_{l}} \in \Omega^{2}\left(\mathbb{R}^{2} ; \operatorname{ad}\left(Q_{\mathrm{GL}(2, \mathbb{C})}\right)\right)
$$

and with the Higgs field $\Phi_{l}$ given by

$$
\Phi_{l}=\left.\phi_{*}\right|_{\mathfrak{m}^{\mathbb{C}}(\mathrm{SL}(2, \mathbb{C}))}\left(\Phi_{1}^{a p p}\right)
$$

Assume, moreover, that the norm of the infinitesimal deformation $\phi_{*}$ satisfies a Lipschitz condition, in other words it holds that

$$
\left\|\phi_{*}(M)\right\|_{\mathfrak{s p}(4, \mathbb{C})} \leq C\|M\|_{\mathfrak{s l}(2, \mathbb{C})}
$$


for $M \in \mathfrak{s l}(2, \mathbb{C})$. In fact, the norms considered above are equivalent to the $C_{0}$-norm. Restricting these norms to $\mathfrak{s o}(2, \mathbb{C})$ and $\mathfrak{m}^{\mathbb{C}}(\mathrm{SL}(2, \mathbb{R}))$ respectively, we may deduce that the error in curvature is still described by the inequality

$$
\left\|* F_{A_{l}^{a p p}}^{\perp}+*\left[\Phi_{l}^{a p p} \wedge\left(\Phi_{l}^{a p p}\right)^{*}\right]\right\|_{C^{0}} \leq k_{l} R^{\delta^{\prime \prime}}
$$

for a (different) real constant $k_{l}$, which still does not depend on the parameter $R>0$.

In summary, using an embedding $\phi: \operatorname{SL}(2, \mathbb{R}) \hookrightarrow \operatorname{Sp}(4, \mathbb{R})$ with the properties described above, we may extend the approximate solution $\left(A_{1}^{a p p}, \Phi_{1}^{a p p}\right)$ to take an approximate $\operatorname{Sp}(4, \mathbb{C})$ pair $\left(A_{l}, \Phi_{l}\right)$ over $X_{1}$, which agrees with a model solution $\left(A_{l, p}^{\bmod }, \Phi_{l, p}^{\bmod }\right)$ over an annulus $\Omega_{1}^{p}$ around each puncture $p \in D_{1}$; the model solution $\left(A_{l, p}^{\bmod }, \Phi_{l, p}^{\bmod }\right)$ is the extension via $\phi$ of the model $\left(A_{1, p}^{\bmod }, \Phi_{1, p}^{\bmod }\right)$ in $\operatorname{SL}(2, \mathbb{R})$. The pair $\left(A_{l}, \Phi_{l}\right)$ lives in the holomorphic principal $\mathrm{GL}(2, \mathbb{C})$-bundle obtained by extension of structure group via $\phi$, which we shall keep denoting as $\left(E_{1}=\left(\mathbb{E}_{1}, \bar{\partial}_{1}\right), h_{1}\right)$ to ease notation.

Repeating the above considerations for another closed Riemann surface $X_{2}$ of genus $g_{2}$ and $D_{2}=\left\{q_{1}, \ldots, q_{s}\right\}$ a collection of $s$-many distinct points of $X_{2}$, we obtain an approximate $\operatorname{Sp}(4, \mathbb{C})$-pair $\left(A_{r}, \Phi_{r}\right)$ over $X_{2}$, which agrees with a model solution $\left(A_{r, q}^{\bmod }, \Phi_{r, q}^{\bmod }\right)$ over an annulus $\Omega_{2}^{q}$ around each puncture $q \in D_{2}$. This pair lives on the holomorphic principal $\mathrm{GL}(2, \mathbb{C})$-bundle obtained by extension of structure group via another appropriate embedding $\mathrm{SL}(2, \mathbb{R}) \hookrightarrow \mathrm{Sp}(4, \mathbb{R}) ;$ let this hermitian bundle be denoted by $\left(E_{2}=\left(\mathbb{E}_{2}, \bar{\partial}_{2}\right), h_{2}\right)$.

4.5. Gluing of the Riemann surfaces. We begin with a classical result from complex analysis and conformal geometry:

Theorem 4.4 (Schottky's Theorem on Conformal Mappings between Annuli). An annulus $\mathbb{A}_{1}=\left\{z \in \mathbb{C}\left|r_{1}<\right| z \mid<R_{1}\right\}$ on the complex plane can be mapped conformally onto the annulus $\mathbb{A}_{2}=\left\{z \in \mathbb{C}\left|r_{2}<\right| z \mid<R_{2}\right\}$ if and only if $\frac{R_{1}}{r_{1}}=\frac{R_{2}}{r_{2}}$. Moreover, every conformal map $f$ : $\mathbb{A}_{1} \rightarrow \mathbb{A}_{2}$ takes the form $f(z)=\lambda z$ or $f(z)=\frac{\lambda}{z}$, where $\lambda \in \mathbb{C}$ with $|\lambda|=\frac{r_{2}}{r_{1}}$ or $|\lambda|=r_{2} R_{1}$ respectively.

Proof. See p. 35 in [1].

Let us consider the Möbius transformation $f_{\lambda}: \mathbb{A}_{1} \rightarrow \mathbb{A}_{2}$ with $f_{\lambda}(z)=\frac{\lambda}{z}$, where $\lambda \in \mathbb{C}$ with $|\lambda|=r_{2} R_{1}=r_{1} R_{2}$. This is a conformal biholomorphism (equivalently bijective, anglepreserving and orientation-preserving) between the two annuli and the continuous extension of the function $z \mapsto\left|f_{\lambda}(z)\right|$ to the closure of $\mathbb{A}_{1}$ reverses the order of the boundary components. Indeed

- for $|z|=r_{1}:\left|f_{\lambda}(z)\right|=\frac{|\lambda|}{|z|}=\frac{r_{2} R_{1}}{r_{1}}=\frac{r_{1} R_{2}}{r_{1}}=R_{2}$.

- for $|z|=R_{1}:\left|f_{\lambda}(z)\right|=\frac{|\lambda|}{|z|}=\frac{r_{2} R_{1}}{R_{1}}=r_{2}$.

Let two compact Riemann surfaces $X_{1}, X_{2}$ of respective genera $g_{1}, g_{2}$. Choose points $p \in$ $X_{1}, q \in X_{2}$ and local charts around these points $\psi_{i}: U_{i} \rightarrow \Delta\left(0, \varepsilon_{i}\right)$ on $X_{i}$, for $i=1,2$. Now fix positive real numbers $r_{i}<R_{i}<\varepsilon_{i}$ such that the following two conditions are satisfied:

- $\psi_{i}^{-1}\left(\overline{\Delta\left(0, R_{i}\right)}\right) \cap U_{j} \neq \varnothing$, for every $U_{j} \neq U_{i}$ from the complex atlas of $X_{i}$. In other words, we are considering an annulus around each of the $p$ and $q$ contained entirely in the neighborhood of a single chart.

- $\frac{R_{2}}{r_{2}}=\frac{R_{1}}{r_{1}}$ 
Now set

$$
X_{i}^{*}=X_{i} \backslash \psi_{i}^{-1}\left(\overline{\Delta\left(0, r_{i}\right)}\right)
$$

Finally, choose the biholomorphism $f_{\lambda}: \mathbb{A}_{1} \rightarrow \mathbb{A}_{2}$ described in the previous subsection. This biholomorphism is used to glue the two Riemann surfaces $X_{1}, X_{2}$ along the inverse image of the annuli $\mathbb{A}_{1}, \mathbb{A}_{2}$ on the surfaces, using the biholomorphism

$$
g_{\lambda}: \Omega_{1}=\psi_{1}^{-1}\left(\mathbb{A}_{1}\right) \rightarrow \Omega_{2}=\psi_{2}^{-1}\left(\mathbb{A}_{2}\right)
$$

with $g_{\lambda}=\psi_{2}^{-1} \circ f_{\lambda} \circ \psi_{1}$.

Define $X_{\lambda}=X_{1} \#_{\lambda} X_{2}=X_{1}^{*} \amalg X_{2}^{*} / \sim$, where the gluing of $\Omega_{1}$ and $\Omega_{2}$ is performed through the equivalence relation which identifies $y \in \Omega_{1}$ with $w \in \Omega_{2}$ iff $w=g_{\lambda}(y)$. For collections of $s$-many distinct points $D_{1}$ on $X_{1}$ and $D_{2}$ on $X_{2}$, this procedure is assumed to be taking place for annuli around each pair of points $(p, q)$ for $p \in D_{1}$ and $q \in D_{2}$.

The manifold $X_{\lambda}$ is endowed with a complex structure inherited from the complex structures of $X_{1}$ and $X_{2}$ : Indeed, if $\mathcal{A}_{1}, \mathcal{A}_{2}$ are complex atlases for $X_{1}, X_{2}$, then $\left.\left.\mathcal{A}_{1}\right|_{X_{1}^{*}} \cup \mathcal{A}_{2}\right|_{X_{2}^{*}}$ is an atlas for $X_{\lambda}$, since we have chosen the gluing region not to overlap between two different charts on each side. On the glued region $\Omega$, there are two charts $\left(\Omega_{1},\left.\psi_{1}\right|_{\Omega_{1}}\right),\left(\Omega_{2},\left.\psi_{2}\right|_{\Omega_{2}}\right)$, whereas $\varphi_{12}=\psi_{1} \circ \psi_{2}^{-1}: \psi_{2}\left(\Omega_{1} \cap \Omega_{2}\right) \rightarrow \psi_{1}\left(\Omega_{1} \cap \Omega_{2}\right)$ is actually $\varphi_{12} \equiv f_{\lambda}: \mathbb{A}_{1} \rightarrow \mathbb{A}_{2}$.

If $X_{1}, X_{2}$ are orientable and orientations are chosen for both, since $f_{\lambda}$ is orientation preserving we obtain a natural orientation on the connected sum $X_{1} \# X_{2}$ which coincides with the given ones on $X_{1}^{*}$ and $X_{2}^{*}$.

Therefore, $X_{\#}=X_{1} \# X_{2}$ is a Riemann surface of genus $g_{1}+g_{2}+s-1$, the complex connected sum, where $g_{i}$ is the genus of the $X_{i}$ and $s$ is the number of points in $D_{1}$ and $D_{2}$. Its complex structure however is heavily dependent on the parameters $p_{i}, q_{i}, \lambda$.

4.6. Gluing of the bundles. For the Riemann surfaces $X_{1}, X_{2}$ as considered in $\S 4.4$, their connected sum $X_{\#}=X_{1} \# X_{2}$ is constructed by gluing annuli around the points $p_{i}$ of $D_{1}$, with annuli around the points $q_{i}$ of $D_{2}$, as described in $\S 4.5$. Moreover, for the pairs $\left(A_{l}, \Phi_{l}\right)$ and $\left(A_{r}, \Phi_{r}\right)$ defined in $\S 4.4$ we make the following important assumption:

Assumption 4.5. The model solutions satisfy $\left(A_{l, p}^{\bmod }, \Phi_{l, p}^{\bmod }\right)=-\left(A_{r, q}^{\bmod }, \Phi_{r, q}^{\bmod }\right)$ for each pair of points $(p, q)$.

Given this assumption, now notice that for the smooth bundles $\left(\mathbb{E}_{1}, \nabla_{l}:=A_{l}+\Phi_{l}+\Phi_{l}^{*}\right)$ and $\left(\mathbb{E}_{2}, \nabla_{r}:=A_{r}+\Phi_{r}+\Phi_{r}^{*}\right)$, the model flat connections will coincide. Let $\nabla:=\nabla_{l}=-\nabla_{r}$ denote this flat connection over the annuli; we can then fix an identification of these flat bundles over the annuli to get a new bundle $\mathbb{E}_{\#}$ as follows:

Let $\Omega_{1}$ be the annulus on $X_{1}$ for any point $p \in D_{1}$ and pick coordinates $z$ around $p$ with $z(p)=0$. Let $V_{1} \cup V_{2}$ an open covering of $\Omega_{1}$, with $V_{1} \cap V_{2}$ having two connected components, say $\left(V_{1} \cap V_{2}\right)^{+}$and $\left(V_{1} \cap V_{2}\right)^{-}$. For a loop $\gamma$ in $\Omega_{1}$ around $p$ take transition functions

$$
g_{1}^{z}(x)=\left\{\begin{array}{cc}
1, & z \in\left(V_{1} \cap V_{2}\right)^{-} \\
\operatorname{hol}\left(\gamma, \nabla_{l}\right), & z \in\left(V_{1} \cap V_{2}\right)^{+}
\end{array}\right.
$$

Similarly, let $\Omega_{2}$ be the annulus on $X_{2}$ for any point $q \in D_{2}$ and pick coordinates $w$ around $q$ with $w(q)=0$. For a loop $\delta$ in $\Omega_{2}$ around $q$ take transition functions

$$
g_{2}^{w}(x)= \begin{cases}\operatorname{hol}\left(\delta, \nabla_{r}\right), & w \in\left(V_{1} \cap V_{2}\right)^{-} \\ 1, & w \in\left(V_{1} \cap V_{2}\right)^{+}\end{cases}
$$


Using an orientation reversing isometry to glue the annuli $\Omega_{1}$ and $\Omega_{2}$ in constructing the connected sum, the region $\left(V_{1} \cap V_{2}\right)^{+}$of $\Omega_{1}$ is glued together with the region $\left(V_{1} \cap V_{2}\right)^{-}$of $\Omega_{2}$. The gluing of the Riemann surfaces is realized along the curve $z w=\lambda$, thus we have

$$
\frac{d z}{z}=-\frac{d w}{w}
$$

on the annuli. Now from Assumption 4.5, $\nabla_{l}=-\nabla_{r}$, and so there is defined a 1-cocycle on $\Omega:=\Omega_{1} \sim \Omega_{2}$ by $g(s):=g_{1}(z)=g_{2}\left(\frac{\lambda}{z}\right)$, since $w=\frac{\lambda}{z}$ for a point $s \in \Omega$. This is repeated for each pair of points $(p, q)$. We may use this identification of the cocycles to define a bundle isomorphism $\left.\left.\mathbb{E}_{1}\right|_{\Omega_{1}} \stackrel{\sim}{\rightarrow} \mathbb{E}_{2}\right|_{\Omega_{2}}$ and use this isomorphism to glue the bundles over $\Omega$ for every pair $(p, q)$ to define the connected sum bundle $\mathbb{E}_{1} \# \mathbb{E}_{2}$.

Remark 4.6. We can alternatively glue the bundles by picking a globally trivial frame on each side, flat with respect to the unitary connection $A$ but not for $\nabla$. Indeed for such a frame for $A_{l}$ and $A_{r}$ glue $\left(\Omega_{1} \times \mathbb{C}^{2}\right) \amalg\left(\Omega_{2} \times \mathbb{C}^{2}\right)$ under the identification map $(z, u) \mapsto(w, v)$ with $w=\frac{\lambda}{z}$ and $u=v$.

4.7. Gluing the connections and hermitian metrics. The pairs $\left(A_{l}, \Phi_{l}\right),\left(A_{r}, \Phi_{r}\right)$ agree over neighborhoods around the points in the divisors $D_{1}$ and $D_{2}$, with $A_{l}=A_{r}=0$ and with $\Phi_{l}(z)=-\Phi_{r}(w)$, thus there is a suitable frame for $\nabla$ over which the hermitian metrics are both described by the identity matrix and so they are constant in particular. Set $\left(A_{p, q}^{\bmod }, \Phi_{p, q}^{\bmod }\right):=\left(A_{l, p}^{\bmod }, \Phi_{l, p}^{\bmod }\right)=-\left(A_{r, q}^{\bmod }, \Phi_{r, q}^{\bmod }\right)$. We can glue the pairs $\left(A_{l}, \Phi_{l}\right),\left(A_{r}, \Phi_{r}\right)$ together to get an approximate solution of the $\operatorname{Sp}(4, \mathbb{R})$-Hitchin equations:

$$
\left(A_{R}^{a p p}, \Phi_{R}^{a p p}\right):=\left\{\begin{array}{cc}
\left(A_{l}, \Phi_{l}\right), & \text { over } X_{1} \backslash X_{2} \\
\left(A_{p, q}^{\bmod }, \Phi_{p, q}^{\bmod }\right) & \text { over } \Omega \text { around each pair of points }(p, q), \\
\left(A_{r}, \Phi_{r}\right) & \text { over } X_{2} \backslash X_{1}
\end{array}\right.
$$

considered on the bundle $\left(\mathbb{E}_{1} \# \mathbb{E}_{2}, h_{\#}\right)$ over the complex connected sum $X_{\#}:=X_{1} \# X_{2}$.
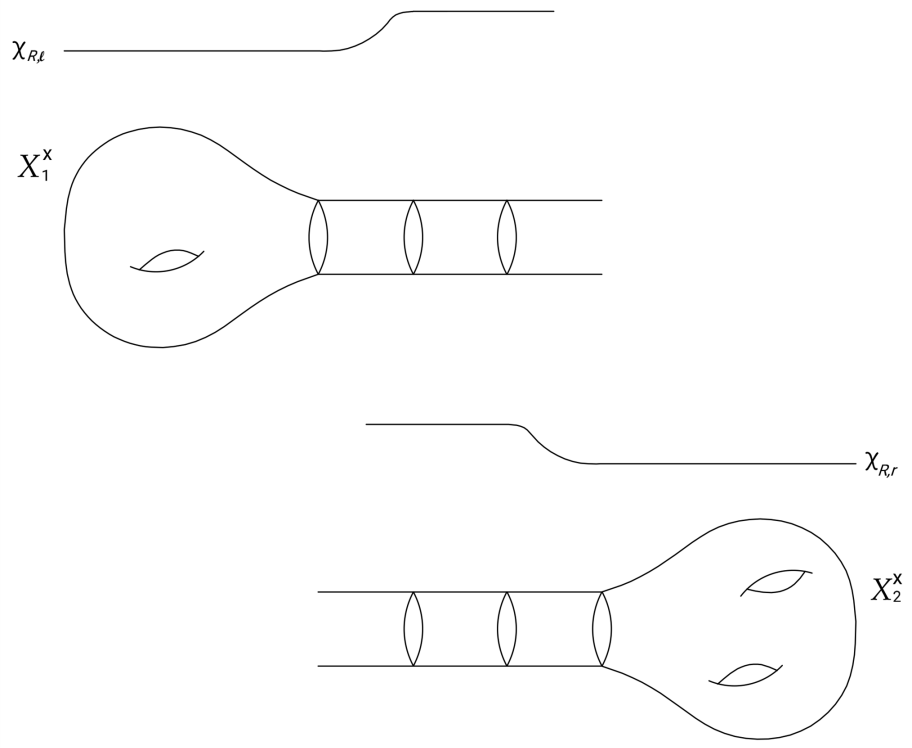

Figure 2. Constructing approximate solutions over $X_{1}^{\times}$and $X_{2}^{\times}$. 


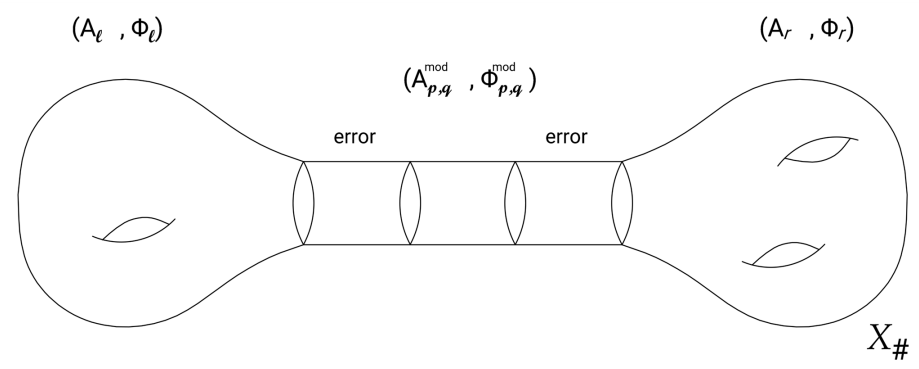

Figure 3. $\left(A_{R}^{a p p}, \Phi_{R}^{a p p}\right)$ over the complex connected sum $X_{\#}$.

By construction, $\left(A_{R}^{a p p}, \Phi_{R}^{a p p}\right)$ is a smooth pair on $X_{\#}$, complex gauge equivalent to an exact solution of the Hitchin equations by a smooth gauge transformation defined over all of $X_{\#}$. It satisfies the second equation, while the first equation is satisfied up to an error which we have good control of:

Lemma 4.7. The approximate solution $\left(A_{R}^{a p p}, \Phi_{R}^{a p p}\right)$ to the parameter $0<R<1$ satisfies

$$
\left\|* F_{A_{R}^{a p p}}+*\left[\Phi_{R}^{a p p},-\tau\left(\Phi_{R}^{a p p}\right)\right]\right\|_{C^{0}\left(X^{\times}\right)} \leq C R^{\delta^{\prime \prime}}
$$

for some constants $\delta^{\prime \prime}>0$ and $C=C\left(\delta^{\prime \prime}\right)$, which do not depend on $R$.

Proof. Follows from Lemma 4.3; take $C:=\max \left\{C_{l}, C_{r}\right\}$, for $C_{l}, C_{r}$ the constants appearing in the bound of the error for the approximate solutions constructed over each of the Riemann surfaces $X_{1}$ and $X_{2}$.

4.8. The representations $\phi_{i r r}$ and $\psi$. In this subsection, we see that the Assumption 4.5 we made for the model pairs can be achieved by taking particular representations from $\operatorname{SL}(2, \mathbb{R})$ into $\operatorname{Sp}(4, \mathbb{R})$.

The irreducible representation $\phi_{i r r}: S L(2, \mathbb{R}) \hookrightarrow S p(4, \mathbb{R})$. Let $\left(A_{1}^{a p p}, \Phi_{1}^{a p p}\right)$ over $X_{1}$ be the approximate $\mathrm{SL}(2, \mathbb{C})$-pair in parameter $R>0$, as was considered in $\S 4.4$, which agrees with the model pair

$$
A_{1}^{\bmod }=0, \Phi_{1}^{\bmod }=\left(\begin{array}{cc}
C & 0 \\
0 & -C
\end{array}\right) \frac{d z}{z}
$$

for $C \in \mathbb{R}$, over an annulus in $z$-coordinates around a point $p \in D_{1}$.

The embedding $\phi_{i r r}$ defined by $(2.4)$ extends to give an embedding $\phi_{i r r}: \operatorname{SL}(2, \mathbb{C}) \hookrightarrow$ $\operatorname{Sp}(4, \mathbb{C})$. For the Lie algebra of $\operatorname{SL}(2, \mathbb{C}), \mathfrak{s l}(2, \mathbb{C})=\left\{\left(\begin{array}{cc}a & b \\ c & -a\end{array}\right) \mid a, b, c \in \mathbb{C}\right\}$, we may use a Cartan basis for the Lie algebra to determine the infinitesimal deformation, $\phi_{i r r}: \mathfrak{s l}(2, \mathbb{C}) \rightarrow$ $\mathfrak{s p}(4, \mathbb{C})$ with

$$
\phi_{i r r} *\left(\left(\begin{array}{cc}
a & b \\
c & -a
\end{array}\right)\right)=\left(\begin{array}{cccc}
3 a & -\sqrt{3} b & 0 & 0 \\
-\sqrt{3} c & a & 0 & 2 b \\
0 & 0 & -3 a & \sqrt{3} c \\
0 & 2 c & \sqrt{3} b & -a
\end{array}\right)
$$

We now notice that $\phi_{\text {irr }}(\mathrm{SO}(2))$ lies in a copy of $\mathrm{U}(2) \hookrightarrow \mathrm{Sp}(4, \mathbb{R})$, that is

$$
\mathrm{U}(2) \cong\left\{\left(\begin{array}{cc}
A & B \\
-B & A
\end{array}\right) \mid A^{T} A+B^{T} B=I_{2}, A^{T} B-B^{T} A=0\right\} .
$$


In other words, copies of a maximal compact subgroup of $\operatorname{SL}(2, \mathbb{R})$ are mapped into copies of a maximal compact subgroup of $\operatorname{Sp}(4, \mathbb{R})$. Furthermore, one can check that for $A \in \mathfrak{s l}(2, \mathbb{C})$ :

$$
\left\|\phi_{i r r *}(A)\right\|_{\mathfrak{s p}(4, \mathbb{C})}=10\|A\|_{\mathfrak{s}(2, \mathbb{C})}
$$

As was described in $\S 4.4, \phi_{i r r}$ can be now used to extend $\operatorname{SL}(2, \mathbb{C})$-data to $\operatorname{Sp}(4, \mathbb{C})$-data $\left(A_{l}, \Phi_{l}\right)$, where in this case, it is $A_{l}=0$ and

$$
\Phi_{l}=\phi_{\text {irr } *} \mid \mathfrak{m}^{\mathbb{C}(\operatorname{SL}(2, \mathbb{C}))}\left(\Phi_{1}^{a p p}\right)=\left(\begin{array}{cccc}
3 C & 0 & 0 & 0 \\
0 & C & 0 & 0 \\
0 & 0 & -3 C & 0 \\
0 & 0 & 0 & -C
\end{array}\right) \frac{d z}{z}
$$

over the annulus on $X_{1}$ in $z$-coordinates around the point $p$.

The representation $\psi: S L(\mathscr{2}, \mathbb{R}) \times S L(2, \mathbb{R}) \hookrightarrow S p(4, \mathbb{R})$. Let $\left(A_{2,1}^{a p p}, \Phi_{2,1}^{a p p}\right),\left(A_{2,2}^{a p p}, \Phi_{2,2}^{a p p}\right)$ over $X_{2}$ be two approximate $\mathrm{SL}(2, \mathbb{C})$-pairs in parameter $R>0$, which agree respectively with the model pairs

$$
A_{2,1}^{\bmod }=0, \Phi_{2,1}^{\bmod }=\left(\begin{array}{cc}
-3 C & 0 \\
0 & 3 C
\end{array}\right) \frac{d z}{z} \text { and } A_{2,2}^{\bmod }=0, \Phi_{2,2}^{\bmod }=\left(\begin{array}{cc}
-C & 0 \\
0 & C
\end{array}\right) \frac{d z}{z}
$$

for the same real parameter $C \in \mathbb{R}$ considered in defining the pair $\left(A_{1}^{a p p}, \Phi_{1}^{a p p}\right)$ over $X_{1}$ above, over an annulus in $w$-coordinates around a point $q \in D_{2}$.

We extend $\operatorname{SL}(2, \mathbb{C}) \times \mathrm{SL}(2, \mathbb{C})$-data into $\operatorname{Sp}(4, \mathbb{C})$ using the homomorphism $\psi$ defined by (2.5). Take the extension of the embedding $\psi$ into $\mathrm{SL}(2, \mathbb{C}) \times \mathrm{SL}(2, \mathbb{C})$, and now the infinitesimal deformation of this homomorphism is given by $\psi_{*}: \mathfrak{s l}(2, \mathbb{C}) \times \mathfrak{s l}(2, \mathbb{C}) \hookrightarrow \mathfrak{s p}(4, \mathbb{C})$ with

$$
\psi_{*}\left(\left(\begin{array}{cc}
a & b \\
c & -a
\end{array}\right),\left(\begin{array}{cc}
e & f \\
g & -e
\end{array}\right)\right)=\left(\begin{array}{cccc}
a & 0 & b & 0 \\
0 & e & 0 & f \\
c & 0 & -a & 0 \\
0 & g & 0 & -e
\end{array}\right)
$$

We may still check that $\psi(\mathrm{SO}(2) \times \mathrm{SO}(2))$ is a copy of $\mathrm{U}(2)$. On the other hand, a norm on the space $\mathfrak{s l}(2, \mathbb{C}) \times \mathfrak{s l}(2, \mathbb{C})$ is given by

$$
\psi(A, B)=\|A\|+\|B\|
$$

and we check that

$$
\left\|\psi_{*}(A, B)\right\|_{\mathfrak{s p}(4, \mathbb{C})}=\|(A, B)\|_{\mathfrak{s l}(2, \mathbb{C}) \times \mathfrak{s l}(2, \mathbb{C})}=\|A\|_{\mathfrak{s l}(2, \mathbb{C})}+\|B\|_{\mathfrak{s l}(2, \mathbb{C})}
$$

and so the map $\psi_{*}$ at the level of Lie algebras is an isometry. Therefore, $\psi$ extends to give an embedding $\psi: \mathrm{SO}(2, \mathbb{C}) \times \mathrm{SO}(2, \mathbb{C}) \hookrightarrow \mathrm{GL}(2, \mathbb{C})$, and so we may use the infinitesimal deformation $\psi_{*}$ to extend the $\operatorname{SL}(2, \mathbb{C}) \times \operatorname{SL}(2, \mathbb{C})$-data $\left(\left(A_{2,1}^{a p p}, \Phi_{2,1}^{a p p}\right),\left(A_{2,2}^{a p p}, \Phi_{2,2}^{a p p}\right)\right)$ to an $\operatorname{Sp}(4, \mathbb{C})$-pair $\left(A_{r}, \Phi_{r}\right)$, with $A_{r}=0$ and Higgs field $\Phi_{r}$ given by

$$
\Phi_{r}=\psi_{*} \mid \mathfrak{m}^{\mathbb{C}}(\operatorname{SL}(2, \mathbb{R})) \times \mathfrak{m}^{\mathbb{C}}(\operatorname{SL}(2, \mathbb{R}))\left(\Phi_{2,1}^{a p p}, \Phi_{2,2}^{a p p}\right)=\left(\begin{array}{cccc}
-3 C & 0 & 0 & 0 \\
0 & -C & 0 & 0 \\
0 & 0 & 3 C & 0 \\
0 & 0 & 0 & C
\end{array}\right) \frac{d z}{z}
$$

over the annulus on $X_{2}$ in $w$-coordinates around the point $q$. 


\section{Correcting An APproximate solution to AN EXACT SOlUtion}

5.1. The contraction mapping argument. A standard strategy, due largely to C. Taubes [37], for correcting an approximate solution to an exact solution of gauge-theoretic equations involves studying the linearization of a relevant elliptic operator. In the Higgs bundle setting, the linearization of the Hitchin operator was described in [25] and furthermore in [36] for solutions to the $\mathrm{SL}(2, \mathbb{C})$-self-duality equations over a noded surface. We are going to use this analytic machinery to correct our approximate solution to an exact solution over the complex connected sum of Riemann surfaces. We next summarize the strategy to be followed in the forthcoming sections:

For the complex connected sum $X_{\#}$ consider the nonlinear $G$-Hitchin operator at a pair $(A, \Phi) \in \Omega^{1}\left(X_{\#}, E_{H}\left(\mathfrak{h}^{\mathbb{C}}\right)\right) \oplus \Omega^{1,0}\left(X_{\#}, E_{H}\left(\mathfrak{g}^{\mathbb{C}}\right)\right):$

$$
\mathcal{H}(A, \Phi)=\left(F(A)-[\Phi, \tau(\Phi)], \bar{\partial}_{A} \Phi\right)
$$

Moreover, consider the orbit map

$$
\gamma \mapsto \mathcal{O}_{(A, \Phi)}(\gamma)=g^{*}(A, \Phi)=\left(g^{*} A, g^{-1} \Phi g\right)
$$

for $g=\exp (\gamma)$ and $\gamma \in \Omega^{0}\left(X_{\#}, E_{H}(\mathfrak{h})\right)$, where $H \subset G$ is a maximal compact subgroup.

Therefore, correcting the approximate solution $\left(A_{R}^{a p p}, \Phi_{R}^{a p p}\right)$ to an exact solution of the $G$ Hitchin equations accounts to finding a point $\gamma$ in the complex gauge orbit of $\left(A_{R}^{a p p}, \Phi_{R}^{a p p}\right)$, for which $\mathcal{H}\left(g^{*}\left(A_{R}^{a p p}, \Phi_{R}^{a p p}\right)\right)=0$. However, since we have seen that the second equation is satisfied by the pair $\left(A_{R}^{a p p}, \Phi_{R}^{a p p}\right)$ and since the condition $\bar{\partial}_{A} \Phi=0$ is preserved under the action of $\mathcal{G}_{H}$, we actually seek for a solution $\gamma$ to the following equation

$$
\mathcal{F}_{R}(\gamma):=p r_{1} \circ \mathcal{H} \circ \mathcal{O}_{\left(A_{R}^{a p p}, \Phi_{R}^{a p p}\right)}(\exp (\gamma))=0
$$

For a Taylor series expansion of this operator

$$
\mathcal{F}_{R}(\gamma)=\operatorname{pr}_{1} \mathcal{H}\left(A_{R}^{a p p}, \Phi_{R}^{a p p}\right)+L_{\left(A_{R}^{a p p}, \Phi_{R}^{a p p}\right)}(\gamma)+Q_{R}(\gamma)
$$

where $Q_{R}$ includes the quadratic and higher order terms in $\gamma$, we can then see that $\mathcal{F}_{R}(\gamma)=0$ if and only if $\gamma$ is a fixed point of the map:

$$
\begin{aligned}
T: H_{B}^{2}\left(X_{\#}\right) & \rightarrow H_{B}^{2}\left(X_{\#}\right) \\
\gamma & \mapsto-G_{R}\left(\mathcal{H}\left(A_{R}^{a p p}, \Phi_{R}^{a p p}\right)+Q_{R}(\gamma)\right)
\end{aligned}
$$

where we denoted $G_{R}:=L_{\left(A_{R}^{a p p}, \Phi_{R}^{a p p}\right)}^{-1}$.

The problem then reduces to showing that the mapping $T$ is a contraction of the open ball $B_{\rho_{R}}$ of radius $\rho_{R}$ in $H_{R}^{2}\left(X_{\#}\right)$, since then from Banach's fixed point theorem there will exist a unique $\gamma$ such that $T(\gamma)=\gamma$, i.e. such that $\mathcal{F}_{R}(\gamma)=0$. In particular, one needs to show that:

(1) $T$ is a contraction defined on $B_{\rho_{R}}$ for some $\rho_{R}$, and

(2) $T$ maps $B_{\rho_{R}}$ to $B_{\rho_{R}}$

In order to perform the above described contraction mapping argument, we need to show the following:

i: The linearized operator at the approximate solution $L_{\left(A_{R}^{a p p}, \Phi_{R}^{a p p}\right)}$ is invertible.

ii: There is an upper bound for the inverse operator $G_{R}=L_{\left(A_{R}^{a p p}, \Phi_{R}^{a p p}\right)}^{-1}$ as an operator $L^{2}\left(r^{-1} d r d \theta\right) \rightarrow L^{2}\left(r^{-1} d r d \theta\right)$. 
iii: There is an upper bound for the inverse operator $G_{R}=L_{\left(A_{R}^{a p p}, \Phi_{R}^{a p p}\right)}^{-1}$ also when viewed as an operator $L^{2}\left(r^{-1} d r d \theta\right) \rightarrow H_{B}^{2}\left(X_{\#}, r^{-1} d r d \theta\right)$.

iv: We can control a Lipschitz constant for $Q_{R}$, i.e. there exists a constant $C>0$ such that

$$
\left\|Q_{R}\left(\gamma_{1}\right)-Q_{R}\left(\gamma_{0}\right)\right\|_{L^{2}} \leq C \rho\left\|\gamma_{1}-\gamma_{0}\right\|_{H_{B}^{2}}
$$

for all $0<\rho \leq 1$ and $\gamma_{0}, \gamma_{1} \in B_{\rho}$, the closed ball of radius $\rho$ around 0 in $H_{B}^{2}\left(X_{\#}\right)$.

5.2. The Linearization operator $L_{(A, \Phi)}$. We first need to characterize the linearization operator $L_{(A, \Phi)}$ in general, before considering this for the particular approximate pair $\left(A_{R}^{a p p}, \Phi_{R}^{a p p}\right)$ that we have constructed. The differential of the $G$-Hitchin operator at a pair $(A, \Phi) \in$ $\Omega^{1}\left(X_{\#}, E_{H}\left(\mathfrak{h}^{\mathbb{C}}\right)\right) \oplus \Omega^{1,0}\left(X_{\#}, E_{H}\left(\mathfrak{g}^{\mathbb{C}}\right)\right)$ is described by

$$
D \mathcal{H}\left(\begin{array}{c}
\dot{A} \\
\dot{\Phi}
\end{array}\right)=\left(\begin{array}{cc}
d_{A} & {[\Phi,-\tau(\cdot)]+[\cdot,-\tau(\Phi)]} \\
{[\cdot, \Phi]} & \bar{\partial}_{A}
\end{array}\right)\left(\begin{array}{l}
\dot{A} \\
\dot{\Phi}
\end{array}\right)
$$

Moreover, the differential at $g=I d$ of the orbit map $\mathcal{O}_{(A, \Phi)}$ is

$$
\Lambda_{(A, \Phi)} \gamma=\left(\bar{\partial}_{A} \gamma-\partial_{A} \gamma^{*},[\Phi, \gamma]\right)
$$

and so when $\gamma \in \Omega^{0}\left(X_{\#}, E_{H}(\mathfrak{h})\right)$ :

$$
\Lambda_{(A, \Phi)} \gamma=\left(\bar{\partial}_{A} \gamma-\partial_{A} \gamma,[\Phi, \gamma]\right)
$$

Therefore,

$$
\left(D \mathcal{H} \circ \Lambda_{(A, \Phi)}\right)(\gamma)=\left(\begin{array}{c}
\left(\partial_{A} \bar{\partial}_{A}-\bar{\partial}_{A} \partial_{A}\right) \gamma+[\Phi,-\tau([\Phi, \gamma])+[[\Phi, \gamma],-\tau(\Phi)]] \\
{\left[\bar{\partial}_{A} \gamma-\partial_{A} \gamma, \Phi\right]+\bar{\partial}_{A}[\Phi, \gamma]}
\end{array}\right)
$$

Now, take

$$
\begin{aligned}
D \mathcal{F}(\gamma): & =D\left(p r_{1} \circ \mathcal{H} \circ \mathcal{O}_{(A, \Phi)}\right)(\gamma)=D \mathcal{H} \circ \Lambda_{(A, \Phi)}(\gamma) \\
& =\left(\partial_{A} \bar{\partial}_{A}-\bar{\partial}_{A} \partial_{A}\right) \gamma+[\Phi,-\tau([\Phi, \gamma])+[[\Phi, \gamma],-\tau(\Phi)]]
\end{aligned}
$$

and consider the operator $M_{\Phi}: \Omega^{0}\left(X_{\#}, E_{H}(\mathfrak{h})\right) \rightarrow \Omega^{0}\left(X_{\#}, E_{H}(\mathfrak{h})\right)$ defined by

$$
M_{\Phi} \gamma:=-[\Phi,[\tau(\Phi), \gamma]]+[\tau(\Phi),[\Phi, \gamma]]
$$

for $\Phi \in \Omega^{1}\left(X_{\#}, E_{H^{\mathbb{C}}}\left(\mathfrak{m}^{\mathbb{C}}\right)\right)$. Then from the identities

$$
\begin{aligned}
2 \bar{\partial}_{A} \partial_{A} & =F(A)-i * \Delta_{A} \\
2 \partial_{A} \bar{\partial}_{A} & =F(A)+i * \Delta_{A} \\
{[\Phi, \tau([\Phi, \gamma])] } & =-[\Phi,[\tau(\Phi), \gamma]]
\end{aligned}
$$

we may deduce that $\left(i * \Delta_{A}+M_{\Phi}\right)(\gamma)=D \mathcal{F}(\gamma)$. (For the first two identities see Propositions $1.421,1.422$ in [27]; the third identity is derived by direct calculations). Now define

$$
L_{(A, \Phi)}:=\Delta_{A}-i * M_{\Phi}: \Omega^{0}\left(X_{\#}, i E_{H}(\mathfrak{h})\right) \rightarrow \Omega^{0}\left(X_{\#}, i E_{H}(\mathfrak{h})\right)
$$

The following lemma first observed by C. Simpson in [33] provides that the linearization operator $L_{(A, \Phi)}$ is nonnegative. The proof given here is a modification of the proof of the analogous statement for the case of $\mathrm{SL}(2, \mathbb{C})$ given in $[25]$.

Lemma 5.1. For $\gamma \in \Omega^{0}\left(X_{\#}, E_{H}(\mathfrak{h})\right)$

$$
\left\langle-i * M_{\Phi} \gamma, \gamma\right\rangle=4|[\Phi, \gamma]|^{2} \geq 0
$$


Proof. Fixing a local holomorphic coordinate $z$, write $\Phi=\varphi d z$ and $\tau(\Phi)=-\varphi^{*} d \bar{z}$. Then $[\tau(\Phi),[\Phi, \gamma]]=\left[\varphi^{*},[\varphi, \gamma]\right] d z \wedge d \bar{z}$ and $-[\Phi,[\tau(\Phi), \gamma]]=\left[\varphi,\left[\varphi^{*}, \gamma\right]\right] d z \wedge d \bar{z}$. Altogether, we may write

$$
M_{\Phi} \gamma=\left(\left[\varphi^{*},[\varphi, \gamma]\right]+\left[\varphi,\left[\varphi^{*}, \gamma\right]\right]\right) d z \wedge d \bar{z}
$$

The compact real form $\tau: \mathfrak{g}^{\mathbb{C}} \rightarrow \mathfrak{g}^{\mathbb{C}}$ induces an ad-invariant inner product on $\mathfrak{g}^{\mathbb{C}}$, thus we get $\left\langle\left[\varphi^{*},[\varphi, \gamma]\right], \gamma\right\rangle=|[\varphi, \gamma]|^{2}$ as well as $\left\langle\left[\varphi,\left[\varphi^{*}, \gamma\right]\right], \gamma\right\rangle=\left|\left[\varphi^{*}, \gamma\right]\right|^{2}=|[\varphi, \gamma]|^{2}$. Finally, since $2 i * 1=-d z \wedge d \bar{z}$, we get $\left\langle M_{\Phi} \gamma, i * \gamma\right\rangle=|[\varphi, \gamma]|^{2}|d z \wedge d \bar{z}|^{2}=4|[\varphi, \gamma]|^{2}$.

The following corollary is now immediate:

Corollary 5.2. If $\gamma \in \Omega^{0}\left(X_{\#}, E_{H}(\mathfrak{h})\right)$, then

$$
\left\langle L_{(A, \Phi)} \gamma, \gamma\right\rangle_{L^{2}}=\left\|d_{A} \gamma\right\|_{L^{2}}^{2}+4\|[\Phi, \gamma]\|_{L^{2}}^{2} \geq 0
$$

In particular, $L_{(A, \Phi)} \gamma=0$ if and only if $d_{A} \gamma=[\Phi, \gamma]=0$.

5.3. Cylindrical Dirac-type operators and the Cappell-Lee-Miller gluing theorem. A very useful method when dealing with surgery problems in gauge theory over manifolds with very long necks involves the study of the space of eigenfunctions corresponding to small eigenvalues (low eigensolutions) of a self-adjoint Dirac type operator on such a manifold (see [9], [28], [40]). For our purposes we will make use of the Cappell-Lee-Miller gluing theorem from [9] and its generalization to small perturbations of constant coefficient operators due to L. Nicolaescu in [28]. In the latter article, a family of manifolds $M_{T}$ for $T_{0} \leq T \leq \infty$ is considered, each containing a long cylindrical neck of length $\sim T=|\log R|$, obtained by gluing of two disjoint manifolds $M_{T}^{ \pm}$along the boundaries of a pair of cylindrical ends. A self-adjoint first-order Dirac-type operator $\mathfrak{D}_{T}$ is then considered on a hermitian vector bundle over each manifold $M_{T}$.

The Cappell-Lee-Miller gluing theorem asserts that under suitable assumptions, the operator $\mathfrak{D}_{T}$ admits two types of eigenvalues, namely those of order of decay $O\left(T^{-1}\right.$ ) (large eigenvalues) and those of order of decay $o\left(T^{-1}\right)$ (small eigenvalues). For $T \rightarrow \infty$, the subspace of $L^{2}$ spanned by the eigenvectors to small eigenvalues is "parameterized" by the kernel of the limiting operator $\mathfrak{D}_{\infty}$. This way, the Dirac operator $\mathfrak{D}_{T}$ has no small eigenvalues, if the limiting operator $\mathfrak{D}_{\infty}$ is invertible. This is the set-up also considered in [36].

We define the family of differential operators we will be considering; precise definitions on $\mathbb{Z}_{2}$-graded Dirac-type operators on cylindrical vector bundles can be found in [28]:

Definition 5.3. Let $\hat{E} \rightarrow \hat{N}$ be a $\mathbb{Z}_{2}$-graded cylindrical hermitian vector bundle. A first order partial differential operator $\mathfrak{D}: C^{\infty}(\hat{E}) \rightarrow C^{\infty}(\hat{E})$ is called a $\mathbb{Z}_{2}$-graded cylindrical Dirac-type operator if with respect to the $\mathbb{Z}_{2}$-grading of $\hat{E}$, it takes the form

$$
\mathfrak{D}=\left(\begin{array}{cc}
0 & \mathcal{D}^{*} \\
\mathcal{D} & 0
\end{array}\right)
$$

such that along the cylindrical end $\mathcal{D}=G(d \tau-D)$ for a self-adjoint Dirac-type operator $D: C^{\infty}\left(E^{+}\right) \rightarrow C^{\infty}\left(E^{+}\right)$.

Recall that the Dirac-type condition asserts that the square $D^{2}$ has the same principal symbols as a Laplacian. $D$ is independent of the longitudinal coordinate $\tau$ along the necks.

For our purposes, we will need to use the perturbed operator $\mathfrak{D}+\mathfrak{B}=\left(\begin{array}{cc}0 & \mathcal{D}+B \\ \mathcal{D}^{*}+B^{*} & 0\end{array}\right)$, where $B$ is an exponentially decaying operator of order 0 ; that means there exists a pair of 
constants $C, \lambda>0$ for which

$$
\sup \{|B(x)| \mid x \in[\tau, \tau+1] \times N\} \leq C e^{-\lambda|\tau|}
$$

for all $\tau \in \mathbb{R}^{+}$.

We can then use the orientation preserving diffeomorphism $\varphi$ to obtain for each $T>0$ the manifold $N_{T}$ by attaching the region $\hat{N}_{1} \backslash(T+1, \infty) \times N_{1}$ to the region $\hat{N}_{2} \backslash(-\infty,-T-1) \times N_{2}$ using the orientation preserving identification

$$
\begin{aligned}
{[T+1, T+2] \times N_{1} } & \rightarrow[-T-2,-T-1] \times N_{2} \\
(\tau, x) & \mapsto(\tau-2 T-3, \varphi(x))
\end{aligned}
$$

The $\mathbb{Z}_{2}$-graded cylindrical hermitian vector bundles $\hat{E}_{i}$ can be similarly glued together providing a $\mathbb{Z}_{2}$-graded hermitian vector bundle $E_{T}=E_{T}^{+} \oplus E_{T}^{-}$over the manifold $N_{T}$. Moreover, the cylindrical operators $\mathfrak{D}_{i}$ combine to give a $\mathbb{Z}_{2}$-graded Dirac-type operator $\mathfrak{D}_{T}$ on the bundle $E_{T}$. For a pair of perturbed operators, we can also obtain a perturbed Dirac-type operator defined on the bundle $E_{T}$; let us still denote this by $\mathfrak{D}_{T}$ and write such an operator as

$$
\mathfrak{D}_{T}=\left(\begin{array}{cc}
0 & \mathcal{D}_{T}^{*} \\
\mathcal{D}_{T} & 0
\end{array}\right)
$$

Consider also $\mathfrak{D}_{i, \infty}:=\mathfrak{D}_{i}+\mathfrak{B}_{i}$ for $i=1,2$ and write

$$
\mathfrak{D}_{i, \infty}=\left(\begin{array}{cc}
0 & \mathcal{D}_{i, \infty}^{*} \\
\mathcal{D}_{i, \infty} & 0
\end{array}\right)
$$

We are going to need one last piece of notation to introduce:

Definition 5.4. Let $\hat{E}$ a cylindrical vector bundle over the cylindrical manifold $\hat{N}$. We define the extended $L^{2}$ space $L_{\text {ext }}^{2}(\hat{N}, \hat{E})$ as the space of all sections $\hat{u}$ of $\hat{E}$, such that there exists an $L^{2}$ section $u_{\infty}$ of $E$ satisfying

$$
\hat{u}-\pi^{*} u_{\infty} \in L^{2}(N, E)
$$

The section $u_{\infty}$ is uniquely determined by $\hat{u}$, thus the so-called asymptotic trace map is welldefined

$$
\begin{aligned}
\partial_{\infty}: L_{\text {ext }}^{2}(\hat{N}, \hat{E}) & \rightarrow L^{2}(N, E) \\
\hat{u} & \mapsto u_{\infty}
\end{aligned}
$$

The following theorem is the version of the Cappell-Lee-Miller gluing theorem, which we are going to apply. For a proof see [28], §5.B:

Theorem 5.5 (S. Cappell-R. Lee-E. Miller, L. Nicolaescu). Let $\mathfrak{D}_{i, \infty}$ be a pair of $\mathbb{Z}_{2 \text {-graded }}$ Dirac-type operators on the cylindrical vector bundles $\hat{E}_{i} \rightarrow \hat{N}_{i}$ for $i=1,2$ as was defined above. Suppose that the kernel $K_{i}^{+} \subseteq L_{\text {ext }}^{2}\left(\hat{N}_{i}, \hat{E}_{i}\right)$ of the operator $\mathcal{D}_{i, \infty}$ is trivial for $i=1,2$. Then there exist a $T_{0}>0$ and a constant $C>0$ such that the operator $\mathcal{D}_{T}^{*} \mathcal{D}_{T}$ is bijective for all $T>T_{0}$ and admits a bounded inverse $\left(\mathcal{D}_{T}^{*} \mathcal{D}_{T}\right)^{-1}: L^{2}\left(N_{T}, E_{T}^{+}\right) \rightarrow L^{2}\left(N_{T}, E_{T}^{+}\right)$with

$$
\left\|\left(\mathcal{D}_{T}^{*} \mathcal{D}_{T}\right)^{-1}\right\|_{\mathcal{L}\left(L^{2}, L^{2}\right)} \leq C T^{2}
$$




\section{The LineARIZATION OPERATOR FOR AN APPROXIMATE SOLUTION}

6.1. The elliptic complex over the complex connected sum. Into our setting, note that a punctured neighborhood on a Riemann surface can be also thought of, using a cylindrical coordinate transformation, as a half cylinder attached to the surface, and also an annulus in the real parameter $R$ can be thought of as a finite tube of length $\sim T=|\log R|$. Thus, the gluing of two punctured Riemann surfaces as we described it in $\S 4.5$ can be thought of as the gluing of two Riemann surfaces with cylindrical ends to get a smooth surface with a finite number of long Euclidean cylinders of length $2|\log R|$, one for each $p \in \mathfrak{p}$. For our approximate solution $\left(A_{R}^{a p p}, \Phi_{R}^{a p p}\right)$ constructed over $X_{\#}$ with $0<R<1$ and $T=-\log R$, consider the elliptic complex:

$$
\begin{aligned}
0 \rightarrow \Omega^{0}\left(X_{\#}, E_{H}\left(\mathfrak{h}^{\mathbb{C}}\right)\right) \stackrel{L_{1, T}}{\longrightarrow} \Omega^{1}\left(X_{\#}, E_{H}\left(\mathfrak{h}^{\mathbb{C}}\right)\right) \oplus \Omega^{1,0}\left(X_{\#}, E_{H}\left(\mathfrak{g}^{\mathbb{C}}\right)\right) \\
\stackrel{L_{2, T}}{\longrightarrow} \Omega^{2}\left(X_{\#}, E_{H}\left(\mathfrak{h}^{\mathbb{C}}\right)\right) \oplus \Omega^{2}\left(X_{\#}, E_{H}\left(\mathfrak{g}^{\mathbb{C}}\right)\right) \rightarrow 0
\end{aligned}
$$

where

$$
L_{1, T} \gamma=\left(d_{A_{R}^{a p p}} \gamma,\left[\Phi_{R}^{a p p}, \gamma\right]\right)
$$

is the linearization of the complex gauge group action and

$$
L_{2, T}(\alpha, \varphi)=D \mathcal{H}(\alpha, \varphi)=\left(\begin{array}{c}
d_{A_{R}^{a p p} \alpha} \alpha\left[\Phi_{R}^{a p p},-\tau(\varphi)\right]+\left[\varphi,-\tau\left(\Phi_{R}^{a p p}\right)\right] \\
\bar{\partial}_{A_{R}^{a p p} \varphi} \varphi\left[\varphi, \Phi_{R}^{a p p}\right]
\end{array}\right)
$$

is the differential of the Hitchin operator considered in $\S 5.2$.

Note that in general it does not hold that $L_{2, T} L_{1, T}=\left[F_{A_{R}^{a p p}, \gamma}\right]+\left[\left[\Phi_{R}^{a p p},-\tau\left(\Phi_{R}^{a p p}\right)\right], \gamma\right]=$ 0 , since $\left(A_{R}^{a p p}, \Phi_{R}^{a p p}\right)$ need not be an exact solution. Decomposing $\Omega^{*}\left(X_{\#}, E_{H}\left(\mathfrak{g}^{\mathbb{C}}\right)\right)$ into forms of even, respectively odd total degree, we may introduce the $\mathbb{Z}_{2}$-graded Dirac-type operator

$$
\mathfrak{D}_{T}:=\left(\begin{array}{cc}
0 & L_{1, T}^{*}+L_{2, T} \\
L_{1, T}+L_{2, T}^{*} & 0
\end{array}\right)
$$

on the closed surface $X_{\#}$.

As $R \searrow 0$, the curve $X_{\#}$ degenerates to a noded surface $X_{\#}^{\times}$(equivalently the cylindrical neck of $X_{\#}$ extends infinitely). For the cut-off functions $\chi_{R}$ that we considered in obtaining the approximate pair $\left(A_{R}^{a p p}, \Phi_{R}^{a p p}\right)$, their support will tend to be empty as $R \searrow 0$, i.e. the "error regions" disappear along with the neck $N$, thus $\left(A_{R}^{a p p}, \Phi_{R}^{a p p}\right) \rightarrow\left(A_{0}, \Phi_{0}\right)$ uniformly on compact subsets with

$$
\left(A_{0}^{a p p}, \Phi_{0}^{a p p}\right)= \begin{cases}\left(A_{l}, \Phi_{l}\right), & X_{l} \backslash N \\ \left(A_{r}, \Phi_{r}\right), & X_{r} \backslash N\end{cases}
$$

an exact solution with the holonomy of the associated flat connection in $G$.

For $T=\infty$ the elliptic complex for the exact solution $\left(A_{0}^{a p p}, \Phi_{0}^{a p p}\right)$ gives rise to the Diractype operator

$$
\mathfrak{D}_{\infty}=\left(\begin{array}{cc}
0 & L_{1}^{*}+L_{2} \\
L_{1}+L_{2}^{*} & 0
\end{array}\right)
$$

We now describe the map $L_{1}+L_{2}^{*}$ more closely. Using the Hodge $*$-operator we can identify

$$
\Omega^{2}\left(X_{\#}^{\times}, E_{H}\left(\mathfrak{h}^{\mathbb{C}}\right)\right) \cong \Omega^{0}\left(X_{\#}^{\times}, E_{H}\left(\mathfrak{h}^{\mathbb{C}}\right)\right) \text { and } \Omega^{2}\left(X_{\#}^{\times}, E_{H}\left(\mathfrak{g}^{\mathbb{C}}\right)\right) \cong \Omega^{0}\left(X_{\#}^{\times}, E_{H}\left(\mathfrak{g}^{\mathbb{C}}\right)\right)
$$


as well as $\Omega^{1}\left(X_{\#}^{\times}, E_{H}\left(\mathfrak{h}^{\mathbb{C}}\right)\right) \cong \Omega^{0,1}\left(X_{\#}, E_{H}\left(\mathfrak{g}^{\mathbb{C}}\right)\right)$ via the projection $A \mapsto \pi^{0,1} A$. We further identify

$$
\left(\gamma_{1}, \gamma_{2}\right) \in \Omega^{0}\left(X_{\#}^{\times}, E_{H}\left(\mathfrak{h}^{\mathbb{C}}\right)\right) \oplus \Omega^{0}\left(X_{\#}^{\times}, E_{H}\left(\mathfrak{h}^{\mathbb{C}}\right)\right)
$$

with $\psi_{1}=\gamma_{1}+i \gamma_{2} \in \Omega^{0}\left(X_{\#}^{\times}, E_{H}\left(\mathfrak{g}^{\mathbb{C}}\right)\right)$. The operator $L_{1}+L_{2}^{*}$ can be now expressed as the map $L_{1}+L_{2}^{*}$ :

$$
\begin{aligned}
\Omega^{0}\left(X_{\#}^{\times}, E_{H}\left(\mathfrak{g}^{\mathbb{C}}\right)\right) \oplus \Omega^{0}\left(X_{\#}^{\times}, E_{H}\left(\mathfrak{g}^{\mathbb{C}}\right)\right) & \rightarrow \Omega^{0,1}\left(X_{\#}^{\times}, E_{H}\left(\mathfrak{g}^{\mathbb{C}}\right)\right) \oplus \Omega^{1,0}\left(X_{\#}^{\times}, E_{H}\left(\mathfrak{g}^{\mathbb{C}}\right)\right) \\
\left(\psi_{1}, \psi_{2}\right) & \mapsto\left(\begin{array}{c}
\bar{\partial}_{A_{0}^{a p p}} \psi_{1}+\left[\psi_{2},-\tau\left(\Phi_{0}^{a p p}\right)\right] \\
\partial_{A_{0}^{a p p} \psi_{2}}+\left[\psi_{1}, \Phi_{0}^{a p p}\right]
\end{array}\right)
\end{aligned}
$$

6.2. $\mathfrak{D}_{\infty}$ is an exponentially small perturbation of a cylindrical operator. Consider the operator $\hat{\mathfrak{D}}_{\infty}:=\left(\begin{array}{cc}0 & \hat{L}_{1}^{*}+\hat{L}_{2} \\ \hat{L}_{1}+\hat{L}_{2}^{*} & 0\end{array}\right)$ arising similarly from the elliptic complex for some model solution $\left(A^{\text {mod }}, \Phi^{\text {mod }}\right)$ replacing $\left(A_{0}^{a p p}, \Phi_{0}^{a p p}\right)$, and for which

$$
\left(A^{\mathrm{mod}}, \Phi^{\mathrm{mod}}\right)=\left(0, \varphi \frac{d z}{z}\right)
$$

along each cylindrical neck. The operator $\hat{\mathfrak{D}}_{\infty}$ is in fact cylindrical. Indeed, introducing the complex coordinate $\zeta=\tau+i \theta$, we have the identities $d \tau=-\frac{d r}{r}, d \theta=-d \theta, \frac{d z}{z}=-d \zeta$, and $\frac{d \bar{z}}{\bar{z}}=-d \bar{\zeta}$. Hence the operator $\hat{L}_{1}+\hat{L}_{2}^{*}$ (as well as the operator $\hat{L}_{1}^{*}+\hat{L}_{2}$ similarly) can be written as a cylindrical differential operator $\hat{L}_{1}+\hat{L}_{2}^{*}: \frac{\sqrt{2}}{2} G\left(\partial_{\tau}-D\right)$ with

$$
\left(\psi_{1}, \psi_{2}\right) \mapsto \frac{1}{2}\left(\begin{array}{c}
\partial_{\tau} \psi_{1} d \bar{\zeta} \\
\partial_{\tau} \psi_{2} d \zeta
\end{array}\right)-\left(\begin{array}{c}
\left(\frac{i}{2} \partial_{\theta} \psi_{1}+\left[\psi_{2}, \tau(\varphi)\right]\right) d \bar{\zeta} \\
\left(-\frac{i}{2} \partial_{\theta} \psi_{2}-\left[\psi_{2}, \varphi\right]\right) d \zeta
\end{array}\right)
$$

where

$$
D\left(\psi_{1}, \psi_{2}\right):=2\left(\begin{array}{c}
\frac{i}{2} \partial_{\theta} \psi_{1}+\left[\psi_{2}, \tau(\varphi)\right] \\
-\frac{i}{2} \partial_{\theta} \psi_{2}-\left[\psi_{2}, \varphi\right]
\end{array}\right)
$$

and $G=\left(\psi_{1}, \psi_{2}\right)=\frac{\sqrt{2}}{2}\left(\psi_{1} d \bar{\zeta}, \psi_{2} d \zeta\right)$ denotes Clifford multiplication by $d \tau$.

By construction of the approximate solution $\left(A_{R}^{a p p}, \Phi_{R}^{a p p}\right)$ and the decay described in Lemma 4.1 , one sees that the operator $\mathfrak{D}_{\infty}$ is an exponentially small perturbation of $\hat{\mathfrak{D}}_{\infty}$.

6.3. The space $\operatorname{ker}\left(L_{1}+L_{2}^{*}\right) \cap L_{\text {ext }}^{2}\left(X_{\#}^{\times}\right)$is trivial. We now restrict to the case $G=$ $\operatorname{Sp}(4, \mathbb{R})$ in order to study the space $\operatorname{ker}\left(L_{1}+L_{2}^{*}\right) \cap L_{\text {ext }}^{2}\left(X_{\#}^{\times}\right)$for the operator $\mathfrak{D}_{\infty}$ more closely. We are also taking here into consideration the particular model Higgs field we picked for the $G=\operatorname{Sp}(4, \mathbb{R})$-Hitchin equations coming from the particular embeddings $\phi_{i r r}$ and $\psi$ from (2.4) and (2.5). In other words, we fix

$$
\varphi \equiv \varphi^{\bmod }=\left(\begin{array}{cccc}
3 C & 0 & 0 & 0 \\
0 & C & 0 & 0 \\
0 & 0 & -3 C & 0 \\
0 & 0 & 0 & -C
\end{array}\right)
$$

Moreover, the compact real form on $\varphi$ in this case is $\tau(\varphi)=-\varphi^{*}$. We have the following: 
Proposition 6.1. Let $\left(\psi_{1}, \psi_{2}\right) \in \operatorname{ker}\left(L_{1}+L_{2}^{*}\right) \cap L_{\text {ext }}^{2}\left(X_{\#}^{\times}\right)$. Then its asymptotic trace is described by

$$
\partial_{\infty}\left(\psi_{1}, \psi_{2}\right)=\left(\left(\begin{array}{cccc}
a_{1} & 0 & 0 & 0 \\
0 & d_{1} & 0 & 0 \\
0 & 0 & -a_{1} & 0 \\
0 & 0 & 0 & -d_{1}
\end{array}\right),\left(\begin{array}{cccc}
a_{2} & 0 & 0 & 0 \\
0 & d_{2} & 0 & 0 \\
0 & 0 & -a_{2} & 0 \\
0 & 0 & 0 & -d_{2}
\end{array}\right)\right)
$$

for constants $a_{i}, d_{i} \in \mathbb{C}$, for $i=1,2$.

Proof. By [28], p. 169, the space of asymptotic traces of $\operatorname{ker}\left(L_{1}+L_{2}^{*}\right)$ is a subspace of $\operatorname{ker} D$ with $D$ as defined in (6.1). We will check that the elements of the latter have the asserted form. Consider the Fourier decomposition $\left(\psi_{1}, \psi_{2}\right)=\left(\sum_{j \in \mathbb{Z}} \psi_{1, j} e^{i j \vartheta}, \sum_{j \in \mathbb{Z}} \psi_{2, j} e^{i j \vartheta}\right)$ where

$$
\psi_{i, j} \in \mathfrak{s p}(4, \mathbb{C})=\left\{\left(\begin{array}{cc}
A & B \\
C & -A^{T}
\end{array}\right) \mid A, B, C \in M_{2 \times 2}(\mathbb{C}) ; B^{T}=B, C^{T}=C\right\},
$$

Then the equation $D\left(\psi_{1}, \psi_{2}\right)=0$ is equivalent to the system of linear equations

$$
\left(\begin{array}{c}
-\frac{j}{2} \psi_{1, j}-\left[\varphi^{*}, \psi_{2, j}\right] \\
\frac{j}{2} \psi_{2, j}-\left[\varphi, \psi_{1, j}\right]
\end{array}\right)=0
$$

for $j \in \mathbb{Z}$. Since the Higgs field $\varphi$ is diagonal, the operator $D$ acts invariantly on diagonal, respectively off-diagonal endomorphisms. It therefore suffices to consider these two cases separately.

Case 1. Let $\left(\psi_{1, j}, \psi_{2, j}\right)=\left(\left(\begin{array}{cccc}a_{1, j} & 0 & 0 & 0 \\ 0 & d_{1, j} & 0 & 0 \\ 0 & 0 & -a_{1, j} & 0 \\ 0 & 0 & 0 & -d_{1, j}\end{array}\right),\left(\begin{array}{cccc}a_{2, j} & 0 & 0 & 0 \\ 0 & d_{2, j} & 0 & 0 \\ 0 & 0 & -a_{2, j} & 0 \\ 0 & 0 & 0 & -d_{2, j}\end{array}\right)\right)$, with $a_{i, j}, d_{i, j} \in \mathbb{C}$ for $i=1,2$. Then Equation (6.2) is equivalent to the pair of equations

$$
\frac{j}{2}\left(\begin{array}{cccc}
a_{1, j} & 0 & 0 & 0 \\
0 & d_{1, j} & 0 & 0 \\
0 & 0 & -a_{1, j} & 0 \\
0 & 0 & 0 & -d_{1, j}
\end{array}\right)=\mathbb{O}, \text { for } i=1,2
$$

thus the system has a non-trivial solution if and only if $j=0$. In other words, $\psi_{1}=\psi_{1,0}$ and $\psi_{2}=\psi_{2,0}$ are of the asserted form.

Case 2. Let now $\left(\psi_{1, j}, \psi_{2, j}\right)=\left(\left(\begin{array}{cccc}0 & b_{1, j} & e_{1, j} & f_{1, j} \\ c_{1, j} & 0 & f_{1, j} & g_{1, j} \\ k_{1, j} & l_{1, j} & 0 & -c_{1, j} \\ l_{1, j} & m_{1, j} & -b_{1, j} & 0\end{array}\right),\left(\begin{array}{cccc}0 & b_{2, j} & e_{2, j} & f_{2, j} \\ c_{2, j} & 0 & f_{2, j} & g_{2, j} \\ k_{2, j} & l_{2, j} & 0 & -c_{2, j} \\ l_{2, j} & m_{2, j} & -b_{2, j} & 0\end{array}\right)\right)$

with all entries in $\mathbb{C}$. Then Equation (6.2) reads as the pair of equations

$$
\frac{j}{2}\left(\begin{array}{cccc}
0 & b_{1, j} & e_{1, j} & f_{1, j} \\
c_{1, j} & 0 & f_{1, j} & g_{1, j} \\
k_{1, j} & l_{1, j} & 0 & -c_{1, j} \\
l_{1, j} & m_{1, j} & -b_{1, j} & 0
\end{array}\right)=\left(\begin{array}{cccc}
0 & -2 b_{2, j} \bar{C} & -6 e_{2, j} \bar{C} & -4 f_{2, j} \bar{C} \\
2 c_{2, j} \bar{C} & 0 & -4 f_{2, j} \bar{C} & -2 g_{2, j} \bar{C} \\
6 k_{2, j} \bar{C} & 4 l_{2, j} \bar{C} & 0 & -2 c_{2, j} \bar{C} \\
4 l_{2, j} \bar{C} & 2 m_{2, j} \bar{C} & 2 b_{2, j} \bar{C} & 0
\end{array}\right)
$$


and

$$
-\frac{j}{2}\left(\begin{array}{cccc}
0 & b_{2, j} & e_{2, j} & f_{2, j} \\
c_{2, j} & 0 & f_{2, j} & g_{2, j} \\
k_{2, j} & l_{2, j} & 0 & -c_{2, j} \\
l_{2, j} & m_{2, j} & -b_{2, j} & 0
\end{array}\right)=\left(\begin{array}{cccc}
0 & -2 b_{1, j} C & -6 e_{1, j} C & -4 f_{1, j} C \\
2 c_{1, j} C & 0 & -4 f_{1, j} C & -2 g_{1, j} C \\
6 k_{1, j} C & 4 l_{1, j} C & 0 & -2 c_{1, j} C \\
4 l_{1, j} C & 2 m_{1, j} C & 2 b_{1, j} C & 0
\end{array}\right)
$$

This pair of equations is then equivalent to the equation

$$
\left(\begin{array}{cc}
\frac{j}{2} & 2 \bar{C} \\
-2 C & \frac{j}{2}
\end{array}\right)\left(\begin{array}{l}
b_{1, j} \\
b_{2, j}
\end{array}\right)=\left(\begin{array}{l}
0 \\
0
\end{array}\right)
$$

and seven more similar equations for the $c_{i, j}, e_{i, j}, f_{i, j}, g_{i, j}, k_{i, j}, l_{i, j}, m_{i, j}, i=1,2$. Since $C \neq 0$, we have that the determinant of the $2 \times 2$ matrix in Equation $(6.3)$ is $\left(\frac{j}{2}\right)^{2}+4 C \bar{C}>0$, and so this system has no non-trivial solution for $\left(b_{1, j}, b_{2, j}\right)$; the same is true for the rest seven equations. Therefore, there are no non-trivial off-diagonal elements in ker $D$ and so the only non-trivial elements are of the asserted form in the proposition.

Lemma 6.2. Suppose $\left(\psi_{1}, \psi_{2}\right) \in \operatorname{ker}\left(L_{1}+L_{2}^{*}\right) \cap L_{\text {ext }}^{2}\left(X_{\#}^{\times}\right)$. Then

$$
d_{A_{0}^{a p p}} \psi_{i}=\left[\psi_{i}, \Phi_{0}^{a p p}\right]=\left[\psi_{i},\left(\Phi_{0}^{a p p}\right)^{*}\right]=0
$$

for $i=1,2$.

Proof. See J. Swoboda [36], Lemma 3.11, Step 1.

Proposition 6.3. The operator $L_{1}+L_{2}^{*}$ considered as a densely defined operator on $L_{\text {ext }}^{2}\left(X_{\#}^{\times}\right)$ has trivial kernel.

Proof. Let $\left(\psi_{1}, \psi_{2}\right) \in \operatorname{ker}\left(L_{1}+L_{2}^{*}\right) \cap L_{\text {ext }}^{2}\left(X_{\#}^{\times}\right)$. From Lemma 6.2 we have:

$$
d_{A_{0}^{a p p}} \psi_{i}=\left[\psi_{i}, \Phi_{0}^{a p p}\right]=\left[\psi_{i},\left(\Phi_{0}^{a p p}\right)^{*}\right]=0
$$

for $i=1,2$. We show that $\psi_{1}=0$ by showing that $\gamma:=\psi_{1}+\psi_{1}^{*} \in \Omega^{0}\left(X_{\#}^{\times}, \mathfrak{u}(2)\right)$ and $\delta:=i\left(\psi_{1}-\psi_{1}^{*}\right) \in \Omega^{0}\left(X_{\#}^{\times}, \mathfrak{u}(2)\right)$ both vanish. Choosing a holomorphic coordinate $z$ centered at the node of $X_{\#}^{\times}$, the Higgs field $\Phi_{0}^{a p p}$ in our exact solution is written

$$
\Phi_{0}^{a p p}=\varphi \frac{d z}{z}
$$

with $\varphi \in \mathfrak{m}^{\mathbb{C}}(\operatorname{Sp}(4, \mathbb{R}))=\left\{\left(\begin{array}{cc}A & B \\ B & -A\end{array}\right) \mid A, B \in \mathcal{M}_{2}(\mathbb{C})\right.$ with $\left.A^{T}=A, B^{T}=B\right\}$. We get that $d|\gamma|^{2}=2\left\langle d_{A_{0}^{a p p}} \gamma, \gamma\right\rangle=0$, i.e. $|\gamma|$ is constant on $X_{\#}^{\times}$, as well as that $\gamma(x) \in \operatorname{ker} M_{\varphi(x)}$ for all $x \in X_{\#}^{\times}$, since it is in general $M_{\Phi} \gamma=[\Phi, \tau([\Phi, \gamma])]+[\tau(\Phi),[\Phi, \gamma]]$.

Now, this $\gamma(x) \in \mathfrak{u}(2)$ is hermitian. It has orthogonal eigenvectors for distinct eigenvalues, but even if there are degenerate eigenvalues, it is still possible to find an orthonormal basis of $\mathbb{C}^{4}$ consisting of four eigenvectors of $\gamma(x)$, thus $\mathbb{C}^{4}=E_{\lambda_{1}} \oplus \ldots \oplus E_{\lambda_{4}}$, where $\lambda_{i}$ the eigenvalues of $\gamma(x)$. Assuming that $\gamma(x)$ is non-zero, since $[\varphi(x), \gamma(x)]=0$ it follows that $\varphi(x)$ preserves the eigenspaces of $\gamma(x)$ for all $x \in X_{\#}^{\times}$and so $\langle\varphi(x) v, \varphi(x) w\rangle=\langle v, w\rangle$ for $v, w \in \mathbb{C}^{4}$. In other words, $\varphi(x)$ ought to be an isometry with respect to the usual norm in $\mathbb{C}^{4}$. Equivalently, 
$\varphi(x)$ is unitary for all $x \in X_{\#}^{\times}$. However, for a zero $x_{0} \operatorname{of} \operatorname{det} \Phi=\operatorname{det} \tilde{\varphi}\left(x_{0}\right) \frac{d z^{2}}{z^{2}}$ chosen on the left hand side surface $X_{l}$ of $X_{\#}^{\times}$we see that

$$
\varphi\left(x_{0}\right)=\phi_{i r r *}\left(\begin{array}{cc}
0 & 1 \\
z & 0
\end{array}\right)=\left(\begin{array}{cccc}
0 & -\sqrt{3} & 0 & 0 \\
-\sqrt{3} z & 0 & 0 & 2 \\
0 & 0 & 0 & \sqrt{3} z \\
0 & 2 z & \sqrt{3} & 0
\end{array}\right)
$$

which is not unitary. Therefore, $\gamma=0$.

That $\delta$ vanishes, as well as $\psi_{2}=0$, is proven similarly.

$$
\begin{gathered}
\text { 6.4. Upper bound for } L_{\left(A_{R}^{a p p}, \Phi_{R}^{a p p}\right)} \text { in } H^{2}\left(X_{\#}^{\times}\right) \text {. Define the operator } \\
\qquad \mathcal{D}_{T}:=L_{1, T}+L_{2, T}^{*}
\end{gathered}
$$

The following proposition is an immediate consequence of the Cappell-Lee-Miller theorem (Theorem 5.5) for this operator $\mathcal{D}_{T}$ using the fact that the kernel of the limiting operator $L_{1}+L_{2}^{*}$ is trivial on $L_{\text {ext }}^{2}\left(X_{\#}^{\times}\right)$.

Proposition 6.4. There exist constants $T_{0}>0$ and $C>0$ such that the operator $\mathcal{D}_{T}^{*} \mathcal{D}_{T}$ is bijective for all $T>T_{0}$ and its inverse $\left(\mathcal{D}_{T}^{*} \mathcal{D}_{T}\right)^{-1}: L^{2}\left(X_{\#}\right) \rightarrow L^{2}\left(X_{\#}\right)$ satisfies

$$
\left\|\left(\mathcal{D}_{T}^{*} \mathcal{D}_{T}\right)^{-1}\right\|_{\mathcal{L}\left(L^{2}, L^{2}\right)} \leq C T^{2} .
$$

We are finally in position to imply the existence of the inverse operator $G_{R}=L_{\left(A_{R}^{a p p}, \Phi_{R}^{a p p}\right)}^{-1}$ : $L^{2}\left(X_{\#}\right) \rightarrow L^{2}\left(X_{\#}\right)$ and provide an upper bound for its norm, by adapting the analogous proof from [36] into our case. We first need the following:

Corollary 6.5. There exist constants $T_{0}>0$ and $C>0$ such that for all $T>T_{0}$ and $\gamma \in \Omega^{0}\left(X_{\#}, E_{H}(\mathfrak{h})\right)$ it holds that

$$
\left\|L_{1, T}^{*} L_{1, T} \gamma\right\|_{L^{2}\left(X_{\#}\right)} \geq C T^{-2}\|\gamma\|_{L^{2}\left(X_{\#}\right)}
$$

Proof. The previous proposition provides the existence of constants $T_{0}>0$ and $C>0$ such that for all $T>T_{0}$ and $\gamma \in \Omega^{0}\left(X_{\#}, E_{H}(\mathfrak{h})\right)$ :

$$
\left\|\left(\mathcal{D}_{T}^{*} \mathcal{D}_{T}\right)^{-1} \gamma\right\|_{L^{2}\left(X_{\#}\right)} \leq C T^{2}\|\gamma\|_{L^{2}\left(X_{\#}\right)}
$$

and thus

$$
\left\|\mathcal{D}_{T}^{*} \mathcal{D}_{T} \gamma\right\|_{L^{2}\left(X_{\#}\right)} \geq C T^{-2}\|\gamma\|_{L^{2}\left(X_{\#}\right)}
$$

According to the definition of $\mathcal{D}_{T}$ we have

$$
\begin{aligned}
\mathcal{D}_{T}^{*} \mathcal{D}_{T} & =\left(L_{1, T}+L_{2, T}^{*}\right)^{*}\left(L_{1, T}+L_{2, T}^{*}\right) \\
& =L_{1, T}^{*} L_{1, T}+L_{2, T} L_{1, T}+L_{1, T}^{*} L_{2, T}^{*}+L_{2, T} L_{2, T}^{*}
\end{aligned}
$$

as well as $L_{2, T} L_{1, T} \gamma=\left[F_{A_{R}^{a p p}}, \gamma\right]+\left[\left[\Phi_{R}^{a p p},-\tau\left(\Phi_{R}^{a p p}\right)\right], \gamma\right]$, for sections $\gamma \in \Omega^{0}\left(X_{\#}, E_{H}(\mathfrak{h})\right)$. For parameter $T=-\log R$, Lemma 4.7 provides the estimate

$$
\begin{aligned}
\left\|L_{2, T} L_{1, T} \gamma\right\|_{L^{2}\left(X_{\#}\right)} & \leq C_{1} R^{\delta^{\prime \prime}}\|\gamma\|_{L^{2}\left(X_{\#}\right)} \\
& =C_{1} e^{-\delta^{\prime \prime} T}\|\gamma\|_{L^{2}\left(X_{\#}\right)}
\end{aligned}
$$


for $T$-independent constants $C_{1}, \delta^{\prime \prime}>0$.

Remember that the operator $\mathcal{D}_{T}^{*} \mathcal{D}_{T}$ acts on forms of even total degree. Now, decomposing forms of even total degree into forms of degree zero and degree two, for a 0 -form $\gamma$ we may write $\gamma=\gamma+0$ and thus is

$$
L_{1, T}^{*} L_{1, T} \gamma=\mathcal{D}_{T}^{*} \mathcal{D}_{T} \gamma-L_{2, T} L_{1, T} \gamma
$$

The triangle inequality now provides that

$$
\begin{aligned}
\left\|L_{1, T}^{*} L_{1, T} \gamma\right\|_{L^{2}\left(X_{\#}\right)} & \geq\left\|\mathcal{D}_{T}^{*} \mathcal{D}_{T} \gamma\right\|_{L^{2}\left(X_{\#}\right)}-\left\|L_{2, T} L_{1, T} \gamma\right\|_{L^{2}\left(X_{\#}\right)} \\
& \geq C T^{-2}\|\gamma\|_{L^{2}\left(X_{\#}\right)}-C_{1} e^{-\delta^{\prime \prime} T}\|\gamma\|_{L^{2}\left(X_{\#}\right)},
\end{aligned}
$$

which in turn for sufficiently large $T$ implies the desired inequality.

Proposition 6.6. There exist constants $R_{0}>0$ and $C>0$, such that for all sufficiently small $0<R<R_{0}$ the operator $L_{\left(A_{R}^{a p p}, \Phi_{R}^{a p p}\right)}$ is invertible and satisfies the estimate

$$
\left\|G_{R} \gamma\right\|_{L^{2}\left(X_{\#}\right)} \leq C|\log R|^{2}\|\gamma\|_{L^{2}\left(X_{\#}\right)}
$$

Proof. It suffices to show the statement for the unitarily equivalent operator (which we shall still denote by $\left.L_{\left(A_{R}^{a p p}, \Phi_{R}^{a p p}\right)}\right)$ acting on the space $\Omega^{0}\left(X_{\#}, E_{H}(\mathfrak{h})\right)$ defined after conjugation by the map $\gamma \mapsto i \gamma$. From Corollary 5.2 it follows for all $\gamma \in \Omega^{0}\left(X_{\#}, E_{H}(\mathfrak{h})\right)$ that

$$
\left\langle\left(L_{\left(A_{R}^{a p p}, \Phi_{R}^{a p p}\right)}-L_{1, T}^{*} L_{1, T}\right) \gamma, \gamma\right\rangle=3\left\|\left[\Phi_{R}^{a p p}, \gamma\right]\right\|^{2} \geq 0
$$

Consequently, $L_{\left(A_{R}^{a p p}, \Phi_{R}^{a p p}\right)}-L_{1, T}^{*} L_{1, T}$ is a nonnegative operator. Furthermore, from Corollary 6.5 we obtain the estimate:

$$
\left\|L_{\left(A_{R}^{a p p}, \Phi_{R}^{a p p}\right)} \gamma\right\|_{L^{2}\left(X_{\#}\right)} \geq\left\|L_{1, T}^{*} L_{1, T} \gamma\right\|_{L^{2}\left(X_{\#}\right)} \geq C T^{-2}\|\gamma\|_{L^{2}\left(X_{\#}\right)}
$$

Therefore, the operator $L_{\left(A_{R}^{a p p}, \Phi_{R}^{a p p}\right)}$ is strictly positive, and so invertible, and the norm of its inverse is bounded above by the inverse of the smallest eigenvalue of $L_{\left(A_{R}^{a p p}, \Phi_{R}^{a p p}\right)}$, thus providing the statement of the proposition.

This upper bound for the inverse operator $G_{R}$ is valid also when $G_{R}$ is viewed as an operator $L^{2}\left(X_{\#}, r^{-1} d r d \theta\right) \rightarrow H_{B}^{2}\left(X_{\#}, r^{-1} d r d \theta\right)$, where $H_{B}^{2}\left(X_{\#}\right)$ is the Banach space defined by:

$$
H_{B}^{2}\left(X_{\#}\right):=\left\{\gamma \in L^{2}\left(X_{\#}\right) \mid \nabla_{B} \gamma, \nabla_{B}^{2} \gamma \in L^{2}\left(X_{\#}\right)\right\}
$$

The proof of this statement readily adapts from the proof of Proposition 3.14 and Corollary 3.15 in [36]; we refer the interested reader to this article for the details.

6.5. Lipschitz constants for $Q_{R}$. The orbit map for any Higgs pair $(A, \Phi)$ and any $g=$ $\exp (\gamma)$ with $\gamma \in \Omega^{0}\left(X_{\#}, E_{H}\left(\mathfrak{h}^{\mathbb{C}}\right)\right)$ is given by

$$
\mathcal{O}_{(A, \Phi)}(\gamma)=g^{*}(A, \Phi)=\left(A+g^{-1}\left(\bar{\partial}_{A} g\right)-\left(\partial_{A} g\right) g^{-1}, g^{-1} \Phi g\right)
$$

thus

$$
\begin{aligned}
\exp (\gamma)^{*} A & =A+\left(\bar{\partial}_{A}-\partial_{A}\right) \gamma+R_{A}(\gamma) \\
\exp (-\gamma) \Phi \exp (\gamma) & =\Phi+[\Phi, \gamma]+R_{\Phi}(\gamma)
\end{aligned}
$$

where these reminder terms are

$$
R_{A}(\gamma)=\exp (-\gamma)\left(\bar{\partial}_{A} \exp (\gamma)\right)-\left(\partial_{A} \exp (\gamma)\right) \exp (-\gamma)-\left(\bar{\partial}_{A}-\partial_{A}\right) \gamma
$$


MODEL HIGGS BUNDLES IN EXCEPTIONAL COMPONENTS

$$
R_{\Phi}(\gamma)=\exp (-\gamma) \Phi \exp (\gamma)-[\Phi, \gamma]-\Phi
$$

The Taylor series expansion of the operator $\mathcal{F}_{R}$ is then

$$
\mathcal{F}_{R}(\exp (\gamma))=\operatorname{pr}_{1}\left(\mathcal{H}_{R}(A, \Phi)\right)+L_{R} \gamma+Q_{R} \gamma
$$

with

$$
\begin{aligned}
Q_{R}(\gamma) & :=d_{A}\left(R_{A}(\gamma)\right)+\left[\Phi^{*}, R_{\Phi}(\gamma)\right]+\left[\Phi, R_{\Phi}(\gamma)^{*}\right] \\
& +\frac{1}{2}\left[\left(\left(\bar{\partial}_{A}-\partial_{A}\right) \gamma+R_{A}(\gamma)\right),\left(\left(\bar{\partial}_{A}-\partial_{A}\right) \gamma+R_{A}(\gamma)\right)\right] \\
& +\left[\left([\Phi, \gamma]+R_{\Phi}(\gamma)\right),\left([\Phi, \gamma]+R_{\Phi}(\gamma)\right)^{*}\right]
\end{aligned}
$$

Lemma 6.7. In the above, let $(A, \Phi) \equiv\left(A_{R}^{a p p}, \Phi_{R}^{a p p}\right)$. Then there exists a constant $C>0$ such that

$$
\left\|Q_{R}\left(\gamma_{1}\right)-Q_{R}\left(\gamma_{0}\right)\right\|_{L^{2}\left(X_{\#}\right)} \leq C r\left\|\gamma_{1}-\gamma_{0}\right\|_{H_{B}^{2}\left(X_{\#}\right)}
$$

for all $0<r \leq 1$ and $\gamma_{0}, \gamma_{1} \in B_{r}$, the closed ball of radius $r$ around 0 in $H_{B}^{2}\left(X_{\#}\right)$.

Proof. see [36], Lemma 4.1.

\section{Gluing TheOREMS}

The necessary prerequisites are now in place in order to apply the contraction mapping argument described in $\S 5.1$ and correct the approximate solution constructed into an exact solution of the $\operatorname{Sp}(4, \mathbb{R})$-Hitchin equations.

Theorem 7.1. There exists a constant $0<R_{0}<1$, and for every $0<R<R_{0}$ there exist a constant $\sigma_{R}>0$ and a unique section $\gamma \in H_{B}^{2}\left(X_{\#}, \mathfrak{u}(2)\right)$ satisfying $\|\gamma\|_{H_{B}^{2}\left(X_{\#}\right)} \leq \sigma_{R}$, so that for $g=\exp (\gamma)$ :

$$
\left(A_{\#}, \Phi_{\#}\right)=g^{*}\left(A_{R}^{a p p}, \Phi_{R}^{a p p}\right)
$$

is an exact solution of the $S p(4, \mathbb{R})$-Hitchin equations over the closed surface $X_{\#}$.

Proof. We show that for $\sigma_{R}>0$ sufficiently small, the operator $T$ from $\S 5.1$ defined by $T(\gamma)=-G_{R}\left(\mathcal{H}\left(\left(A_{R}^{a p p}, \Phi_{R}^{a p p}\right)\right)+Q_{R}(\gamma)\right)$ is a contraction of $B_{\sigma_{R}}$, the open ball of radius $\sigma_{R}$. From Proposition 6.6 and Lemma 6.7 we get

$$
\begin{aligned}
\left\|T\left(\gamma_{1}-\gamma_{0}\right)\right\|_{H_{B}^{2}\left(X_{\#}\right)} & =\left\|G_{R}\left(Q_{R}\left(\gamma_{1}\right)-Q_{R}\left(\gamma_{0}\right)\right)\right\|_{H_{B}^{2}\left(X_{\#}\right)} \\
& \leq C(\log R)^{2}\left\|Q_{R}\left(\gamma_{1}\right)-Q_{R}\left(\gamma_{0}\right)\right\|_{L^{2}\left(X_{\#}\right)} \\
& \leq C(\log R)^{2} \sigma_{R}\left\|\gamma_{1}-\gamma_{0}\right\|_{H_{B}^{2}\left(X_{\#}\right)}
\end{aligned}
$$

Let $\varepsilon>0$ and set $\sigma_{R}:=C^{-1}|\log R|^{-2-\varepsilon}$. Then for all $0<R<e^{-1}$ it follows that $C(\log R)^{2} \sigma_{R}<1$ and therefore $T$ is a contraction on the ball of radius $\sigma_{R}$.

Furthermore, since $Q_{R}(0)=0$, using again Proposition 6.6 and Lemma 6.7 we have

$$
\begin{aligned}
\|T(0)\|_{H_{B}^{2}\left(X_{\#}\right)} & =\left\|G_{R}\left(\operatorname{pr}_{1}\left(\mathcal{H}_{R}\left(A_{R}^{a p p}, \Phi_{R}^{a p p}\right)\right)\right)\right\|_{H_{B}^{2}\left(X_{\#}\right)} \\
& \leq C(\log R)^{2}\left\|\operatorname{pr}_{1}\left(\mathcal{H}_{R}\left(A_{R}^{a p p}, \Phi_{R}^{a p p}\right)\right)\right\|_{L^{2}\left(X_{\#}\right)} \\
& \leq C(\log R)^{2} R^{\delta^{\prime \prime}}
\end{aligned}
$$

Thus, when $R_{0}$ is chosen to be sufficiently small, then $\|T(0)\|_{H_{B}^{2}\left(X_{\#}\right)}<\frac{1}{10} \sigma_{R}$, for all $0<R<$ $R_{0}$ and for the above choice of $\sigma_{R}$; thus the ball $B_{\sigma_{R}}$ is mapped to itself by $T$. 
Remark 7.2. The analytic arguments developed in the preceding sections provide also that the Main Theorem 1.1 in [36] also holds for solutions to the $\operatorname{Sp}(4, \mathbb{R})$-Hitchin equations. In particular, we have the following:

Corollary 7.3. Let $\left(\Sigma, J_{0}\right)$ be a Riemann surface with nodes at a finite collection of points $\mathfrak{p} \subset$ $\Sigma$. Let $\left(A_{0}, \Phi_{0}\right)$ be a solution to the $S p(4, \mathbb{R})$-Hitchin equations with logarithmic singularities at $\mathfrak{p}$, which is obtained from a solution to the $S L(2, \mathbb{R})$-Hitchin equations via an embedding $\rho: S L(2, \mathbb{R}) \hookrightarrow S p(4, \mathbb{R})$ that maps a copy of a maximal compact subgroup of $S L(2, \mathbb{R})$ into a maximal compact subgroup of $S p(4, \mathbb{R})$. Suppose that there is a model solution near those nodes which is of the form described in $\S 4.1$. Let $\left(\Sigma, J_{i}\right)$ be a sequence of smooth Riemann surfaces converging uniformly to $\left(\Sigma, J_{0}\right)$. Then, for every sufficiently large $i \in \mathbb{N}$, there exists a smooth solution $\left(A_{i}, \Phi_{i}\right)$ on $\left(\Sigma, J_{i}\right)$, such that $\left(A_{i}, \Phi_{i}\right) \rightarrow\left(A_{0}, \Phi_{0}\right)$ as $i \rightarrow \infty$, uniformly on compact subsets of $\Sigma \backslash \mathfrak{p}$.

Theorem 7.1 now implies that for $\bar{\partial}:=A_{\#}^{0,1}$, the Higgs bundle $\left(E_{\#}:=\left(\mathbb{E}_{\#}, \bar{\partial}\right), \Phi_{\#}\right)$ is a polystable $\operatorname{Sp}(4, \mathbb{R})$-Higgs bundle over the complex connected sum $X_{\#}$. Collecting the steps from the last three sections 4,5,6 we now have our main result:

Theorem 7.4. Let $X_{1}$ be a closed Riemann surface of genus $g_{1}$ and $D_{1}=\left\{p_{1}, \ldots, p_{s}\right\}$ be a collection of s-many distinct points on $X_{1}$. Consider respectively a closed Riemann surface $X_{2}$ of genus $g_{2}$ and a collection of also s-many distinct points $D_{2}=\left\{q_{1}, \ldots, q_{s}\right\}$ on $X_{2}$. Let $\left(E_{1}, \Phi_{1}\right) \rightarrow X_{1}$ and $\left(E_{2}, \Phi_{2}\right) \rightarrow X_{2}$ be parabolic stable $S p(4, \mathbb{R})$-Higgs bundles with corresponding solutions to the Hitchin equations $\left(A_{1}, \Phi_{1}\right)$ and $\left(A_{2}, \Phi_{2}\right)$. Assume that these solutions agree with model solutions $\left(A_{1, p_{i}}^{\bmod }, \Phi_{1, p_{i}}^{\bmod }\right)$ and $\left(A_{2, q_{j}}^{\bmod }, \Phi_{2, q_{j}}^{\bmod }\right)$ near the points $p_{i} \in$ $D_{1}$ and $q_{j} \in D_{2}$, and that the model solutions satisfy $\left(A_{1, p_{i}}^{\bmod }, \Phi_{1, p_{i}}^{\bmod }\right)=-\left(A_{2, q_{j}}^{\bmod }, \Phi_{2, q_{j}}^{\bmod }\right)$, for s-many possible pairs of points $\left(p_{i}, q_{j}\right)$. Then there is a polystable $S p(4, \mathbb{R})$-Higgs bundle $\left(E_{\#}, \Phi_{\#}\right) \rightarrow X_{\#}$, constructed over the complex connected sum of Riemann surfaces $X_{\#}=$ $X_{1} \# X_{2}$, which agrees with the initial data over $X_{\#} \backslash X_{1}$ and $X_{\#} \backslash X_{2}$.

Remark 7.5. In $\S 4.8$ we checked that for the particular parabolic $\operatorname{Sp}(4, \mathbb{R})$-Higgs bundles arising from the representations $\phi_{i r r}$ and $\psi$, the main assumption in the theorem does apply.

Definition 7.6. We call an $\operatorname{Sp}(4, \mathbb{R})$-Higgs bundle constructed by the procedure developed in $\S 4-7$ a hybrid $S p(4, \mathbb{R})$-Higgs bundle.

\section{TOPOLOGICAL INVARIANTS}

In this final section, we identify the connected component of the moduli space $\mathcal{M}^{\max }$ a hybrid Higgs bundle lies, given a choice of stable parabolic ingredients to glue. For this, we need to look at how do the Higgs bundle topological invariants behave under the complex connected sum operation. As an application, we see that under the right initial choices for the gluing data, we can find model Higgs bundles in the exceptional components of the maximal $\operatorname{Sp}(4, \mathbb{R})$-Higgs bundle moduli space; these models are described by the hybrid Higgs bundles of $\S 7$.

8.1. Degree of a connected sum bundle. Let $X_{1}$ and $X_{2}$ be closed Riemann surfaces with divisors $D_{1}$ and $D_{2}$ of $s$-many distinct points on each, and let $V_{1}, V_{2}$ be two parabolic principal $H^{\mathbb{C}}$-bundles over $X_{1}, X_{2}$ respectively. Assume that the underlying smooth bundles $\mathbb{V}_{1}, \mathbb{V}_{2}$ come equipped with adapted hermitian metrics $h_{1}, h_{2}$. Let $\left(\mathbb{V}_{1} \# \mathbb{V}_{2}, h_{\#}\right)$ be the smooth hermitian bundle over the complex connected sum $X_{\#}$ of $X_{1}$ and $X_{2}$. The hermitian metric 
$h_{\#}$ coincides with $h_{1}$ and $h_{2}$ in a neighborhood of $X_{1} \backslash \Omega$ and $X_{2} \backslash \Omega$ respectively, where $\Omega$ is the neck region in the connected sum construction. We have the following:

Proposition 8.1. Let $X_{\#}=X_{1} \# X_{2}$ be the complex connected sum of two closed Riemann surfaces $X_{1}$ and $X_{2}$ with divisors $D_{1}$ and $D_{2}$ of s-many distinct points on each surface, and let $V_{1}, V_{2}$ be parabolic principal $H^{\mathbb{C}}$-bundles over $X_{1}$ and $X_{2}$ respectively. For a parabolic subgroup $P \subset H^{\mathbb{C}}$, a holomorphic reduction $\sigma$ of the structure group of $E$ from $H^{\mathbb{C}}$ to $P$ and an antidominant character $\chi$ of $P$, the following identity holds:

$$
\operatorname{deg}\left(V_{1} \# V_{2}\right)(\sigma, \chi)=\operatorname{pardeg}_{\alpha_{1}}\left(V_{1}\right)(\sigma, \chi)+\operatorname{pardeg}_{\alpha_{2}}\left(V_{2}\right)(\sigma, \chi)
$$

Proof. Consider smooth metrics $\hbar_{1}, \hbar_{2}$ on the principal $H^{\mathbb{C}}$-bundles $V_{1}, V_{2}$ defined over $X_{1}$ and $X_{2}$, which coincide with the adapted metrics $h_{1}, h_{2}$ on $X_{1} \backslash D_{1}, X_{2} \backslash D_{2}$ respectively.

For $v>0$, let $X_{i, v}:=\left\{x \in X_{i} \mid d(x, D) \geq e^{-v}\right\}$ and $B_{i, v}:=X_{i} \backslash X_{i, v}$, for $i=1$, 2. For a holomorphic reduction $\sigma$ and an antidominant character $\chi$, the metrics $\hbar_{i}, h_{i}$ induce metrics $\hbar_{i, L}, h_{i, L}$ on $\left(V_{i}\right)_{\sigma, L}$ with curvature $F_{h_{i}, L}$ and $F_{\hbar_{i}, L}$ respectively. Similarly, the smooth metric $h_{\#}$ on $V_{1} \# V_{2}$ induces a metric $h_{\#, L}$ on $\left(V_{1} \# V_{2}\right)_{\sigma, L}$ with curvature $F_{h_{\#}, L}$. We now have:

$$
\begin{aligned}
& \operatorname{deg}\left(V_{1} \# V_{2}\right)(\sigma, \chi)=\frac{\sqrt{-1}}{2 \pi} \int_{X_{\#}}\left\langle F_{h_{\#}, L}, s_{\sigma}\right\rangle \\
& =\frac{\sqrt{-1}}{2 \pi} \int_{X_{1, v}}\left\langle F_{h_{1}, L}, s_{\sigma}\right\rangle+\frac{\sqrt{-1}}{2 \pi} \int_{X_{2, v}}\left\langle F_{h_{2}, L}, s_{\sigma}\right\rangle+\frac{\sqrt{-1}}{2 \pi} \int_{X_{\#} \backslash\left(X_{1, v} \cup X_{2, v}\right)}\left\langle F_{h_{\#}, L}, s_{\sigma}\right\rangle
\end{aligned}
$$

Now notice:

$$
\frac{\sqrt{-1}}{2 \pi} \int_{X_{1, v}}\left\langle F_{h_{1}, L}, s_{\sigma}\right\rangle=\frac{\sqrt{-1}}{2 \pi} \int_{X_{1}}\left\langle F_{\hbar_{1}, L}, s_{\sigma}\right\rangle-\frac{\sqrt{-1}}{2 \pi} \int_{B_{1, v}}\left\langle F_{h_{1}, L}, s_{\sigma}\right\rangle
$$

and

$$
\frac{\sqrt{-1}}{2 \pi} \int_{X_{1}}\left\langle F_{\hbar_{1}, L}, s_{\sigma}\right\rangle=\operatorname{deg}\left(\mathbb{V}_{1}\right)(\sigma, \chi)
$$

similarly for the integral over $X_{2, v}$. Therefore, for every $v>0$ :

$$
\begin{aligned}
\operatorname{deg}\left(V_{1} \# V_{2}\right)(\sigma, \chi) & =\operatorname{deg}\left(V_{1}\right)(\sigma, \chi)-\frac{\sqrt{-1}}{2 \pi} \int_{B_{1, v}}\left\langle F_{h_{1}, L}, s_{\sigma}\right\rangle+\operatorname{deg}\left(V_{2}\right)(\sigma, \chi) \\
& -\frac{\sqrt{-1}}{2 \pi} \int_{B_{2, v}}\left\langle F_{h_{2}, L}, s_{\sigma}\right\rangle+\frac{\sqrt{-1}}{2 \pi} \int_{X_{\#} \backslash\left(X_{1, v} \cup X_{2, v}\right)}\left\langle F_{h_{\#}, L}, s_{\sigma}\right\rangle
\end{aligned}
$$

Passing to the limit as $v \rightarrow+\infty$, the last integral vanishes, while each integral over $B_{i, v}$ for $i=1,2$ converges to the local term measuring the contribution of the parabolic structure in the definition of the parabolic degree (see Lemma 2.10 in [3]). The desired identity now follows.

Proposition 8.1 implies in particular that the complex connected sum of maximal parabolic $\operatorname{Sp}(4, \mathbb{R})$-Higgs bundles is a maximal (non-parabolic) $\operatorname{Sp}(4, \mathbb{R})$-Higgs bundle. This is the analogue in the language of Higgs bundles of the additivity property for the Toledo invariant from the point of view of fundamental group representations (Proposition 2.13). 
8.2. Model maximal parabolic $\operatorname{Sp}(4, \mathbb{R})$-Higgs bundles. Let $X$ be a compact Riemann surface of genus $g$ and let the divisor $D:=\left\{x_{1}, \ldots, x_{s}\right\}$ of $s$-many distinct points on $X$, assuming that $2 g-2+2 s>0$. Fix a square root of the canonical bundle, that is, a line bundle $L \rightarrow X$, such that $L^{2}=K$ and consider

$$
E=(L \otimes \iota)^{*} \oplus L
$$

where $\iota=\mathcal{O}_{X}(D)$ is the line bundle over the divisor $D$. Assign a parabolic structure on $E$ given by a trivial flag $E_{x_{i}} \supset\{0\}$ and weight $\frac{1}{2}$ for every $x_{i} \in D$. Moreover, for any $q \in H^{0}\left(X, K^{2} \otimes \iota\right)$, let

$$
\theta(q)=\left(\begin{array}{ll}
0 & 1 \\
q & 0
\end{array}\right) \in H^{0}(X, \text { End }(E) \otimes K \otimes \iota)
$$

be the parabolic Higgs field on the parabolic bundle $E$. The authors in [5] show that the pair $(E, \theta(q))$ is a parabolic stable Higgs bundle of parabolic degree zero. Under the non-abelian Hodge correspondence for non-compact curves, there is a tame harmonic metric on the bundle $E$. Moreover, it is shown in [5] that parabolic Higgs bundles of the type $(E, \theta(q))$ defined above, are in 1-1 correspondence with Fuchsian representations of $n$-punctured Riemann surfaces. This also implies that the holonomy of the flat connection on $X$ corresponding to $(E, \theta(q))$ is contained (after conjugation) in $\mathrm{SL}(2, \mathbb{R})$.

As was done in the non-parabolic case [7], we shall use embeddings of $\operatorname{SL}(2, \mathbb{R})$ into $\operatorname{Sp}(4, \mathbb{R})$, in order to obtain model parabolic $\mathrm{Sp}(4, \mathbb{R})$-Higgs bundles:

Example 8.2. Consider the parabolic $\operatorname{Sp}(4, \mathbb{R})$-Higgs bundle $\left(V_{1}, \beta_{1}, \gamma_{1}\right)$ which is induced by the embedding through $\phi_{i r r}$ from $\S 2.8$ of the model parabolic $\operatorname{SL}(2, \mathbb{R})$-Higgs bundle $(E, \theta(q))$. Under the preceding terminology, the bundle $V_{1} \rightarrow X_{1}$ is then described as:

$$
V_{1}=\left(L^{3} \otimes \iota\right) \oplus(L \otimes \iota)^{*}
$$

and it comes equipped with a parabolic structure defined by a trivial flag $\left(V_{1}\right)_{x_{i}} \supset\{0\}$ and weight $\frac{1}{2}$ for every $x_{i} \in D$.

Moreover, $V_{1}$ can be expressed as $V_{1}=N_{1} \oplus N_{1}^{*} K$. Indeed, for $N_{1}=L^{3} \otimes \iota$ we see that:

$$
N_{1}^{*} K=\left(L^{3} \otimes \iota\right)^{*} \otimes K=L^{-3} \otimes \xi \otimes L^{2}=(L \otimes \iota)^{*}
$$

It can be checked that this is a parabolic stable $\mathrm{Sp}(4, \mathbb{R})$-Higgs bundle. Also notice that

$$
\begin{aligned}
\text { par } \operatorname{deg} V_{1} & =\text { par deg }\left(L^{3} \otimes \iota\right)+\text { par } \operatorname{deg}(L \otimes \iota)^{*} \\
& =3 g-3+s+\frac{s}{2}+1-g-s+\frac{s}{2}=2 g-2+s .
\end{aligned}
$$

Therefore, $\left(V_{1}, \beta_{1}, \gamma_{1}\right) \in \mathcal{M}_{\text {par }}^{\max }(X, \operatorname{Sp}(4, \mathbb{R}))$ is a model maximal parabolic $S p(4, \mathbb{R})$-Higgs bundle.

Example 8.3. Consider the parabolic $\operatorname{Sp}(4, \mathbb{R})$-Higgs bundle $\left(V_{2}, \beta_{2}, \gamma_{2}\right)$ which is induced by the embedding through $\phi_{\Delta}$ from $\S 2.8$ of the model parabolic $\operatorname{SL}(2, \mathbb{R})$-Higgs bundle $(E, \theta(q))$. Under the preceding terminology, the bundle $V_{2} \rightarrow X$ is then described as:

$$
V_{2}=L \oplus L
$$

and it comes equipped with a parabolic structure defined by a trivial flag $\left(V_{2}\right)_{x_{i}} \supset\{0\}$ and weight $\frac{1}{2}$ for every $x_{i} \in D$.

Moreover, $V_{2}$ can be expressed as $V_{2}=N_{2} \oplus N_{2}^{*} K$. Indeed, for $N_{2}=L$ we see that:

$$
N_{2}^{*} K=L^{-1} \otimes K=L
$$


It can be checked that this is a parabolic stable $\operatorname{Sp}(4, \mathbb{R})$-Higgs bundle. Also notice that

$$
\text { par } \operatorname{deg} V_{2}=2 p a r \operatorname{deg} L=2\left(g-1+\frac{s}{2}\right)=2 g-2+s
$$

Therefore, $\left(V_{2}, \beta_{2}, \gamma_{2}\right) \in \mathcal{M}_{\text {par }}^{\max }(X, \operatorname{Sp}(4, \mathbb{R}))$ is a model maximal parabolic $S p(4, \mathbb{R})$-Higgs bundle.

In light of Proposition 8.1 we now derive that the polystable hybrid $\operatorname{Sp}(4, \mathbb{R})$-Higgs bundle constructed, $\left(V_{\#}, \Phi_{\#}, h_{\#}, \bar{\partial}\right)$, is maximal:

Proposition 8.4. The hybrid Higgs bundle $\left(V_{\#}, \Phi_{\#}, h_{\#}, \bar{\partial}\right)$ constructed by gluing the maximal parabolic Higgs bundles $\left(V_{1}, \beta_{1}, \gamma_{1}\right)$ and $\left(V_{2}, \beta_{2}, \gamma_{2}\right)$ described above is maximal, i.e. $\operatorname{deg}\left(V_{\#}\right)=$ $2\left(g_{1}+g_{2}+s-1\right)-2=2 g-2$, where $g$ is the genus of the Riemann surface $X_{\#}$, the connected sum of the s-punctured Riemann surfaces $X_{1}$ and $X_{2}$.

8.3. Model Higgs bundles in the exceptional components of $\mathcal{M}^{\max }$. Let $X_{1}=\left\{\phi_{i j}^{1}\right\}$ (resp. $X_{2}=\left\{\phi_{i j}^{2}\right\}$ ) the holomorphic transition functions defining the Riemann surface $X_{1}$ (resp. $X_{2}$ ), with respect to an atlas $\mathcal{A}_{1}\left(\operatorname{resp} . \mathcal{A}_{2}\right)$. Then $\left(\phi_{i j}^{1}\right)^{\prime}$ is nowhere zero. Set

$$
t_{i j}^{1}:=\left.\left(\phi_{i j}^{1}\right)^{\prime} \circ \phi_{i}^{1}\right|_{U_{i} \cap U_{j}}
$$

and now these define the tangent bundle $T_{X_{1}}=\left\{t_{i j}^{1}\right\}$. Since $\frac{1}{t_{i j}^{1}}$ is well-defined, we now get:

$$
K_{X_{1}}=T_{X_{1}}^{*}=\left\{l_{i j}^{1}:=\left(t_{i j}^{1}\right)^{-1}\right\}
$$

and similarly for the Riemann surface $X_{2}$

$$
K_{X_{2}}=T_{X_{2}}^{*}=\left\{l_{i j}^{2}:=\left(t_{i j}^{2}\right)^{-1}\right\}
$$

The transition functions $\phi_{i j}^{1}, \phi_{i j}^{2}$ from the atlas $\mathcal{A}=\left.\left.\mathcal{A}_{1}\right|_{X_{1}^{*}} \cup \mathcal{A}_{2}\right|_{X_{2}^{*}}$ of $X_{\#}$ must agree on the gluing region, the annulus $\Omega$. Thus, $l_{i j}^{1}(x)=l_{i j}^{2}(x)$ over $x \in \Omega$. Considering a cover $V_{1} \cup V_{2}$ of $\Omega$, we can define a line bundle isomorphism $\tilde{l}: V_{1} \cap V_{2} \rightarrow \mathbb{C}^{*}$ and now the 1-cocycles $l_{i j}^{1}, l_{i j}^{2}, \tilde{l}$ define the connected sum canonical bundle

$$
K_{X_{\#}}:=K_{X_{1}} \# K_{X_{2}}
$$

Now, take the maximal parabolic model $\left(V_{1}, \beta_{1}, \gamma_{1}\right)$ described in the previous section. Fix another square root $M_{1}$ of the canonical line bundle $K_{X_{1}}$. Now, define:

$$
\begin{aligned}
W_{1} & =V_{1}^{*} \otimes M_{1}=\left[\left(L_{1}^{3} \otimes \iota\right) \oplus\left(L_{1} \otimes \iota\right)^{*}\right]^{*} \otimes M_{1} \\
& =\left[\left(L_{1} \otimes \iota\right) \oplus\left(L_{1}^{-3} \otimes \xi\right)\right] \otimes M_{1}=\left(K_{X_{1}} \otimes \iota\right) \oplus\left(K_{X_{1}}^{*} \otimes \xi\right)
\end{aligned}
$$

i.e. $W_{1}$ is of the form $\mathcal{L} \oplus \mathcal{L}^{*}$ for $\mathcal{L}:=K_{X_{1}} \otimes \iota$ and also the map $\gamma_{1} \otimes I_{M_{1}^{*}}: W_{1}^{*} \rightarrow W_{1}$ is an isomorphism, which comes from the fact that $\gamma_{1}$ is, as follows of the proof of the Milnor-Wood inequality in the parabolic case.

Therefore, the bundle $W_{1} \rightarrow X_{1}$ is determined by an $\mathrm{O}(2)$-cocycle $\left\{w_{\alpha \beta}^{1}\right\}$ with

$$
\operatorname{det}\left\{w_{\alpha \beta}^{1}\right\}=1 \text {. }
$$

Similarly, for the maximal parabolic model $\left(V_{2}, \beta_{2}, \gamma_{2}\right)$ we fix another square root $M_{2}$ of the canonical line bundle $K_{X_{2}}$ and define:

$$
W_{2}=V_{2}^{*} \otimes M_{2}=\left(L_{2} \oplus L_{2}\right)^{*} \otimes M_{2}=L_{2}^{*} M_{2} \oplus L_{2}^{*} M_{2}
$$


i.e. $W_{2}$ is of the form $\mathcal{L} \oplus \mathcal{L}^{*}$ for $\mathcal{L}:=\mathcal{O}$.

Therefore, the bundle $W_{2} \rightarrow X_{2}$ is determined by an $\mathrm{O}(2)$-cocycle $\left\{w_{\alpha \beta}^{2}\right\}$ with

$$
\operatorname{det}\left\{w_{\alpha \beta}^{2}\right\}=1 \text {. }
$$

Consider 1-cocycles around each puncture $x_{i} \in D$ for the bundles $\mathbb{W}_{1}, \mathbb{W}_{2}$ over the annulus $\Omega \equiv \Omega_{1} \sim \Omega_{2}$

$$
\begin{aligned}
w_{i}: U_{1} \cap U_{2} & \rightarrow \mathrm{GL}(4, \mathbb{C}) \\
x & \mapsto g_{i}^{-1}(x) \cdot m_{i}(x)
\end{aligned}
$$

while $\left\{m_{i}(x)\right\}=M_{i}$. At this point, we are using the 1-cocycles that define the connected sum canonical bundle $K_{X_{\#}}$.

For an induced hermitian metric on $\mathbb{W}_{1}$, using the Gram-Schmidt process one can obtain an orthonormal local frame over $\Omega_{1}$, such that the associated 1-cocycle $\tilde{w}_{1}$ is SO (2)-valued. We may use the isomorphism $\left.\left.\mathbb{W}_{1}\right|_{\Omega_{1}} \stackrel{\simeq}{\rightarrow} \mathbb{W}_{2}\right|_{\Omega_{2}}$ induced by the two isomorphisms between the $V_{i}$ and $M_{i}$ described before, to glue the bundles over $\Omega$ subordinate to the covering $U_{1} \cup U_{2}$. For the 1-cocycle over the connected sum bundle $\mathbb{W}_{1} \# \mathbb{W}_{2}$ we also have:

$$
\operatorname{det}\left\{w_{\alpha \beta}^{\#}\right\}=1
$$

Thus, the first Stiefel-Whitney class $w_{1}\left(W_{\#}\right)$ vanishes, and so $V_{\#}=N_{\#} \oplus N_{\#}^{*} K_{X_{\#}}$ with $N_{\#}=N_{1} \# N_{2}$. Moreover, this provides that the Cayley partner $W_{\#}$ of $V_{\#}$ decomposes as $W_{\#}=L_{\#} \oplus L_{\#}^{-1}$ for some line bundle $L_{\#}$. We thus have established the following:

Proposition 8.5. The hybrid Higgs bundle $\left(V_{\#}, \Phi_{\#}\right)$ constructed by gluing the maximal parabolic Higgs bundles $\left(V_{1}, \beta_{1}, \gamma_{1}\right)$ and $\left(V_{2}, \beta_{2}, \gamma_{2}\right)$ of $\S 8.2$ is maximal with a corresponding Cayley partner $W_{\#}$ for which $w_{1}\left(W_{\#}\right)=0$ and $W_{\#}=L_{\#} \oplus L_{\#}^{-1}$, for some line bundle $L_{\#}$ over $X_{\#}$.

Remark 8.6. Compare this result to Proposition 5.9 in [20], where an analogous property for the Stiefel-Whitney classes of a hybrid representation was established.

The degree of this line bundle $L_{\#}$ fully determines the connected component a hybrid Higgs bundle will lie:

Proposition 8.7. For the line bundle $L_{\#}$ appearing in the decomposition $W_{\#}=L_{\#} \oplus L_{\#}^{-1}$ of the Cayley partner, it is

$$
\operatorname{deg}\left(L_{\#}\right)=\operatorname{par} \operatorname{deg} K_{X_{1}} \otimes \iota_{1}
$$

where $\iota_{1}=\mathcal{O}_{X_{1}}\left(D_{1}\right)$.

Proof. The identity of Proposition 8.1 now applies to provide the computation of the degree for the bundle $N_{\#}$ appearing in the decomposition $V_{\#}=N_{\#} \oplus N_{\#}^{*} K_{X_{\#}}$ :

$$
\begin{aligned}
\operatorname{deg}\left(N_{\#}\right) & =\operatorname{par} \operatorname{deg}\left(L_{1}^{3} \otimes \iota_{1}\right)+\operatorname{par} \operatorname{deg}\left(L_{2}\right) \\
& =3\left(g_{1}-1\right)+s+\frac{s}{2}+g_{2}-1+\frac{s}{2} \\
& =g+2 g_{1}-3+s
\end{aligned}
$$


where $g:=g_{1}+g_{2}+s-1$ is the genus of $X_{\#}$.

Considering $N_{\#} \otimes L_{0}^{-\frac{1}{2}}$ for some $L_{0}=K_{\#}^{\frac{1}{2}}$ now gives

$$
\begin{aligned}
\operatorname{deg}\left(N_{\#} \otimes L_{0}^{-\frac{1}{2}}\right) & =g+2 g_{1}-3+s+1-g \\
& =2 g_{1}+s-2 \\
& =-\chi\left(\Sigma_{1}\right)=\text { par } \operatorname{deg} K_{X_{1}} \otimes \iota_{1}
\end{aligned}
$$

where $\iota_{1}=\mathcal{O}_{X_{1}}\left(D_{1}\right)$.

Therefore, we have constructed a holomorphic vector bundle $V_{\#} \rightarrow X_{\#}$ with $\operatorname{deg}\left(V_{\#}\right)=$ $2 g-2$ and $V_{\#}=N_{\#} \oplus N_{\#}^{*} K_{X_{\#}}$ with $\operatorname{deg}\left(N_{\#} \otimes L_{0}^{-\frac{1}{2}}\right)=2 g_{1}-2+s$, which is odd (resp. even) whenever $s$ is odd (resp. even). The contraction mapping argument developed in $\S 5-7$ will provide a holomorphic structure $\bar{\partial}$ with respect to which $\left(V_{\#}, \bar{\partial}\right)$ is a polystable $\operatorname{Sp}(4, \mathbb{R})$ Higgs bundle. The numerical information we already have for the topological invariants of $V_{\#}$ is preserved and it identifies the connected component of the maximal moduli space the tuple $\left(V_{\#}, \Phi, h_{\#}, \bar{\partial}\right)$ will lie.

Remarks 8.8. (1) The component a hybrid Higgs bundle lies depends on the genera and the number of points in the divisors of the initial Riemann surfaces $X_{1}$ and $X_{2}$ in the construction; there are no extra parameters arising from the deformation of stable parabolic data to model data near these points, or the perturbation argument to correct the approximate solution to an exact solution.

(2) The gluing of two parabolic Higgs bundles of the same type as the model $\left(V_{1}, \beta_{1}, \gamma_{1}\right)$ from Example 8.2 implies that $\operatorname{deg}\left(N_{\#}\right)=3 g-3$. On the other hand, the gluing of two parabolic Higgs bundles of the same type as $\left(V_{2}, \beta_{2}, \gamma_{2}\right)$ from Example 8.3 implies that $\operatorname{deg}\left(N_{\#}\right)=g-1$, as expected.

(3) For a hybrid representation $\rho: \pi_{1}(\Sigma) \rightarrow \mathrm{Sp}(4, \mathbb{R})$, O. Guichard and A. Wienhard in [20] defined an Euler class taking values $e=-\chi\left(\Sigma_{l}\right)[\Sigma] \in H^{2}\left(T^{1} \Sigma, \mathbb{Z}\right)$, where $T^{1} \Sigma$ is the unit tangent bundle of the surface $\Sigma=\Sigma_{l} \cup_{\gamma} \Sigma_{r}$ and $\Sigma_{l}$ is considered to be a surface of genus $1 \leq g_{l} \leq g-1$ and one boundary component, thus its Euler characteristic $\chi\left(\Sigma_{l}\right)=2-2 g_{l}-1=1-2 g_{l}$ is an odd integer within $-2 g+3$ and -1 . In addition to Proposition 19 of [20], which describes a relation between the Stiefel-Whitney classes for maximal $\operatorname{Sp}(4, \mathbb{R})$-Higgs bundles and the Stiefel-Whitney classes for $\operatorname{Sp}(4, \mathbb{R})$-representations, we deduce that in the case of Riemann surfaces with $s=1$ point in the divisors, the degree $\operatorname{deg}\left(L_{\#}\right)$ of the underlying bundle $L_{\#}$ in the decomposition of the Cayley partner $W_{\#}=L_{\#} \oplus L_{\#}^{-1}$ of a hybrid $\operatorname{Sp}(4, \mathbb{R})$-Higgs bundle equals the Euler class $e$ for the hybrid representation, although these invariants live naturally in different cohomology groups.

Since $1 \leq g_{1} \leq g_{1}+g_{2}-1$, it follows that

$$
s \leq \operatorname{deg}\left(N_{\#} \otimes L_{0}^{-\frac{1}{2}}\right) \leq 2 g-s-2
$$

with $s$ an integer between 1 and $g-1$. Therefore, the hybrid Higgs bundles constructed are modeling all exceptional $2 g-3$ connected components of $\mathcal{M}^{\max }(X, \operatorname{Sp}(4, \mathbb{R}))$. These components are fully distinguished by the degree of the line bundle $L_{\#}$ for the hybrid Higgs bundle constructed by gluing. 


\section{REFERENCES}

[1] K. Astala, T. Iwaniec, G. Martin, and J. Onninen. Schottky's theorem on conformal mappings between annuli: a play of derivatives and integrals. Complex analysis and dynamical systems III, 35-39, Contemp. Math. 455, Israel Math. Conf. Proc., Amer. Math. Soc., Providence, RI, 2008.

[2] O. Biquard and P. Boalch. Wild non-abelian Hodge theory on curves. Compos. Math. 140 (2004), no. 1, 179-204.

[3] O. Biquard, O. García-Prada and I. Mundet i Riera. Parabolic Higgs bundles and representations of the fundamental group of a punctured surface into a real group. arXiv:1510.04207.

[4] I. Biswas. Parabolic ample bundles. Math. Ann. 307 (1997), 511-529.

[5] I. Biswas, P. Arés-Gastesi and S. Govindarajan. Parabolic Higgs bundles and Teichmüller spaces for punctured surfaces. Trans. Amer. Math. Soc. 349 (1997), no. 4, 1551-1560.

[6] H. U. Boden and K. Yokogawa. Moduli spaces of parabolic Higgs bundles and $K(D)$ pairs over smooth curves: I. Int. J. Math. 7 (1996), 573-598.

[7] S. B. Bradlow, O. García-Prada and P. B. Gothen. Deformations of maximal representations in $\operatorname{Sp}(4, \mathbb{R})$. Q. J. Math. 63 (2012), no. 4, 795-843.

[8] M. Burger, A. Iozzi and A. Wienhard. Surface group representations with maximal Toledo invariant. Ann. of Math. (2) 172 (2010), no. 1, 517-566.

[9] S. Cappell, R. Lee and E. Miller. Self-adjoint elliptic operators and manifold decompositions. Part I: Low eigenmodes and stretching. Comm. Pure Appl. Math. 49 (1996), 825-866.

[10] B. Collier. Maximal $\operatorname{Sp}(4, \mathbb{R})$ surface group representations, minimal immersions and cyclic surfaces. Geom. Dedicata 180 (2016), 241-285.

[11] K. Corlette. Flat G-bundles with canonical metrics. J. Diff. Geom. 28 (1988), 361-382.

[12] S. K. Donaldson. Twisted harmonic maps and the self-duality equations. Proc. London Math. Soc. (3) 55 (1987), 127-131.

[13] S. Donaldson and P. Kronheimer. The geometry of four-manifolds. Oxford Math. Monographs, Oxford Science Publications, 1990.

[14] L. Foscolo. A gluing construction for periodic monopoles. Int. Math. Res. Not. IMRN (2017), no. 24, 7504-7550.

[15] O. García-Prada, P. B. Gothen and I. Mundet i Riera. Higgs bundles and surface group representations in the real symplectic group. J. Topol. 6 (2013), no. 1, 64-118.

[16] O. García-Prada, P. B. Gothen and I. Mundet i Riera. The Hitchin-Kobayashi correspondence, Higgs pairs and surface group representations. arXiv:0909.4487.

[17] O. García-Prada, P. B. Gothen and V. Muñoz. Betti numbers of the moduli space of rank 3 parabolic Higgs bundles. Mem. Amer. Math. Soc. 187 (2007), no. 879, viii+80 pp.

[18] W. M. Goldman. The symplectic nature of fundamental groups of surfaces. Adv. Math. 54 (1984), no. 2, 200-225.

[19] P. B. Gothen. Components of spaces of representations and stable triples. Topology 40 (2001), no. 4, 823-850.

[20] O. Guichard and A. Wienhard. Topological invariants of Anosov representations. J. Topol. 3 (2010), no. 3, 578-642.

[21] S. He. A gluing theorem for the Kapustin-Witten equations with a Nahm pole. arXiv:1707.06182.

[22] N. J. Hitchin. The self-duality equations on a Riemann surface, Proc. London Math. Soc. (3) 55 (1987), 59-126.

[23] H. Konno. Construction of the moduli space of stable parabolic Higgs bundles on a Riemann surface. J. Math. Soc. Japan 45 (1993), no. 2, 253-276.

[24] G. Kydonakis, H. Sun and L. Zhao. Topological invariants of parabolic G-Higgs bundles. arXiv:1806.00884.

[25] R. Mazzeo, J. Swoboda, H. Weiss and F. Witt. Ends of the moduli space of Higgs bundles. Duke Math. J. 165 (2016), no. 12, 2227-2271.

[26] D. Mumford. Theta characteristics of an algebraic curve. Ann. Sci. École Norm. Sup. 4 (1971), no. 4, 181-192.

[27] L. Nicolaescu. Notes on Seiberg-Witten Theory. Graduate Texts in Mathematics 28, American Mathematical Society, 2000.

[28] L. Nicolaescu. On the Cappell-Lee-Miller gluing theorem. Pacif. J. of Math. 206 (2002), no. 1, 159-185.

[29] S. Ramanan. Global calculus. Graduate Texts in Mathematics 65, American Mathematical Society, 2005.

[30] P. Safari. A gluing theorem for Seiberg-Witten moduli spaces. Ph. D. Thesis, Columbia University, 2000. 
[31] A. H. W. Schmitt. Geometric invariant theory and decorated principal bundles, Zürich Lectures in Advanced Mathematics, European Mathematical Society, 2008.

[32] A. H. W. Schmitt. Moduli for decorated tuples of sheaves and representation spaces for quivers, Proc. Indian Acad. Sci. Math. Sci. 115 (2005), no. 1, 15-49.

[33] C. T. Simpson. Constructing variations of Hodge structures using Yang-Mills theory and applications to uniformization. J. Amer. Math. Soc. 1 (1988), 867-918.

[34] C. T. Simpson. Harmonic bundles on noncompact curves. J. Amer. Math. Soc. 3 (1990), no. 3, 713-770.

[35] C. T. Simpson. Higgs bundles and local systems, Inst. Hautes Études Sci. Publ. Math. 75 (1992), 5-95.

[36] J. Swoboda. Moduli spaces of Higgs bundles on degenerating Riemann surfaces. Adv. Math. 322 (2017), 637-681.

[37] C. H. Taubes. Self-dual Yang-Mills connections over non self-dual 4-manifolds, J. Diff. Geom., 17 (1982), 139-170.

[38] V. G. Turaev. A cocycle of the symplectic first Chern class and Maslov indices. (Russian) Funktsional. Anal. i Prilozhen. 18 (1984), no. 1, 43-48.

[39] M. Wolf. Infinite energy harmonic maps and degeneration of hyperbolic surfaces in moduli space. J. Diff. Geom. 33 (1991), 487-539.

[40] T. Yoshida. Floer homology and splittings of manifolds. Ann. of Math. 134 (1991), 277-324.

Department of Mathematics, University of Illinois at URbana-Champaign

1409 W. Green St, Urbana, IL 61801, USA

E-mail address: kydonak2@illinois.edu 